\title{
Development of Pixel Module Assembly Processes for the Phase 2 Upgrade of the ATLAS Detector and \\ Test Beam Measurements with Diamond Pixel Detectors
}

\section{Dissertation}

zur Erlangung des mathematisch-naturwissenschaftlichen Doktorgrades "Doctor rerum naturalium"

der Georg-August-Universität Göttingen

im Promotionsprogramm ProPhys

der Georg-August University School of Science (GAUSS)

vorgelegt von

Helge Christoph Beck

aus Göttingen

Göttingen, 2019 
Betreuungsausschuss

Prof. Dr. Arnulf Quadt

Prof. Dr. Ariane Frey

\section{Mitglieder der Prüfungskommission:}

$\begin{array}{ll}\text { Referent: } & \text { Prof. Dr. Arnulf Quadt } \\ & \text { II. Physikalisches Institut, Georg-August-Universität Göttingen } \\ \text { Koreferent: } & \text { PD. Dr. Markus Keil } \\ & \text { CERN }\end{array}$

Weitere Mitglieder der Prüfungskommission:

Prof Dr. Stan Lai

II. Physikalisches Institut, Georg-August-Universität Göttingen

Prof. Dr. Ariane Frey

II. Physikalisches Institut, Georg-August-Universität Göttingen

Prof. Dr. Steffen Schumann

Institut für Theoretische Physik, Georg-August-Universität Göttingen

Prof. Dr. Michael Seibt

IV. Physikalisches Institut, Georg-August-Universität Göttingen

Tag der mündlichen Prüfung: 06.02.2020

Referenz: II.Physik-UniGö-Diss-2019/08 


\title{
Development of Pixel Module Assembly Processes for the Phase 2 Upgrade of the ATLAS Detector and Test Beam Measurements with Diamond Pixel Detectors
}

\begin{abstract}
One of the most powerful machines to study particle physics is the Large Hadron Collider (LHC). Starting in 2024, it will be upgraded to the High Luminosity-LHC (HL-LHC) to deliver even more data to measure with high precision Standard Model (SM) physics processes, and to maximise the potential to discover new physics. Higher luminosity implies a higher radiation environment, which poses challenges to the detectors of the experiments. To deal with radiation damage, pile up, and needed read-out speed, ATLAS will replace its current tracking detector with the new Inner Tracker (ITk).

The innermost part of the ITk will be instrumented with silicon pixel detectors. To ensure a high quality production of these modules, tools and processes for the assembly are developed. In this thesis, prototype tools are investigated for their alignment precision of the involved parts, and prototype modules are built for demonstration purposes. After building the modules, they are mechanically and electrically tested. For this purpose, a test stand is required to perform parallel testing of multiple modules, in a temperature controlled environment. The detailed features of the test stand are presented in this thesis, along with the first validation results.

Diamond is investigated as an alternative material to silicon, for developing HL-LHC detector sensors and even more radiation hard applications. Although diamond shows an outstanding radiation hardness, diamond sensors generate weaker signals than silicon ones, when exposed to particles. Furthermore, charge trapping reduces even further the signal in polycrystalline diamond. To overcome these limitations, 3D electrodes in the bulk of the sensor are investigated to reduce the travel distance of the charges. Two pixelated diamond detectors read out with the ATLAS FE-I4 chip are qualified in test beam measurements, one with a planar and one with a 3D electrode geometry. Needed improvements for the pixel layout description in the analysis framework and results for efficiencies are presented in this work.
\end{abstract}





\section{Entwicklung von Prozessen für den Modulbau für das Phase 2 Upgrade des ATLAS Detektors und \\ Teststrahlmessungen mit Diamantpixeldetektoren}

\section{Zusammenfassung}

Der Large Hadron Collider (LHC) ist eines der mächtigsten Werkzeuge, um Teilchenphysik zu betreiben. Ab 2024 wird er zum High Luminosity-LHC (HL-LHC) verbessert, um noch mehr Messdaten zu liefern, damit Prozesse des Standard Models (SM) mit hoher Präzision gemessen werden können und die Möglichkeit, neue Physik zu entdecken, erhöht wird. Eine höhere Luminosität bringt ein höheres Strahlungsumfeld mit sich, das eine Herrausforderung für die Detektoren der Experimente darstellt. Um mit den Strahlenschäden, mehreren Interaktionen pro Kollision, und benötigten Auslesegeschwindigkeiten umgegehen zu können, wird ATLAS seinen Spurdetektor mit dem neuen Inner Tracker (ITk) ersetzen.

Der innerste Teil des ITk benutzt Siliziumpixeldetektoren. Um eine hohe Produktionsqualität dieser Module zu gewährleisten, werden Werkzeuge und Prozesse für den Zusammenbau entwickelt. In dieser Arbeit werden Prototypen der Werkzeuge auf ihre Positionierungspräzision der einzelnen Bauteile untersucht und zu Demonstrationszwecken Prototypmodule gebaut. Nachdem die Module gebaut sind, müssen sie auf ihre mechanische und elektrische Qualität geprüft werde. Für diese Messungen ist ein Teststand in Entwicklung, der parallelisierte Tests für mehrere Module gleichzeitig mit Temperaturkontrolle erlaubt. Dessen Status und Validierungen mit Hilfe eines Prototypsystems werden in dieser Arbeit vorgestellt.

Diamant als Sensormaterialalternative wird für Detektoren am HL-LHC und Umgebungen mit noch höherer Strahlenbelastung untersucht. Obwohl seine Strahlenhärte herausragend ist, wird dafür aber ein kleineres Signal pro Teilchen erzeugt als in Silizium. Es wird weiterhin reduziert durch Ladungseinfang in polykristallinen Diamanten. Um Diamant trotz dieser Beschränkungen nutzen zu können, werden 3D-Elektroden im Material des Sensors untersucht, um die Sammlungsdistanz für die Ladungen zu verkürzen. In Teststrahlmessungen werden zwei Diamantdetektoren, die mit dem ATLAS FE-I4-Chip ausgelesen werden, qualifiziert. Davon hat einer eine planare und einer eine 3D-Elektrodengeometrie. Die nötigen Weiterentwicklungen der Analysesoftware, um die Pixelmatrix zu beschreiben, und Effizienzmessungen werden in dieser Arbeit vorgestellt. 

1. Introduction 1

2. Motivation 3

2.1. The Standard Model . . . . . . . . . . . . . . . . . . . . . . . 3

2.2. Beyond the Standard Model . . . . . . . . . . . . . . . . . . . 4

3. LHC and ATLAS Detector 7

3.1. Large Hadron Collider . . . . . . . . . . . . . . . . . . . . . . 7

3.2. ATLAS Detector . . . . . . . . . . . . . . 8

3.3. HL-LHC and ATLAS Upgrade . . . . . . . . . . . . . . . . . 11

4. Solid State Pixel Detectors 17

4.1. Particle Detection with Solid State Detectors . . . . . . . . . . . . . . 17

4.2. Silicon Sensors . . . . . . . . . . . . . . . . . . . 21

4.3. Diamond Sensors . . . . . . . . . . . . . . . . . . . . 24

4.4. Hybrid Pixel Detectors . . . . . . . . . . . . . . . . . . . . . 29

5. Module Assembly and Testing 31

5.1. Module Assembly . . . . . . . . . . . . . . . . . . . . . . 31

5.2. Module Testing . . . . . . . . . . . . . . . . . . . . . . . . . . 44

6. Test Beam Measurements with Diamond Pixel Modules $\mathbf{5 5}$

6.1. Experimental Setup . . . . . . . . . . . . . . . . . . . 55

6.2. Analysis Strategy . . . . . . . . . . . . . . . . . . 63

6.3. Results . . . . . . . . . . . . . . . . . . . . . . 70

7. Conclusion and Outlook $8 \mathbf{8 7}$

7.1. Module Assembly and Testing for the ATLAS Upgrade . . . . . . . . . . 87

7.2. Test Beam Measurements with Diamond Pixel Modules . . . . . . . . . . 89 


\section{Contents}

Bibliography

Appendices

99

$\begin{array}{ll}\text { A. Appendix } & 101\end{array}$

A.1. Module Testing . . . . . . . . . . . . . . . . . . . . . . . 101

A.2. Test Beam Measurements with Diamond Pixel Detectors . . . . . . . . . . 105 


\title{
CHAPTER 1
}

\author{
Introduction
}

Particle physics is a fascinating field of research. Looking back at the last century, it is impressive how much about the structure of matter was discovered. In the Standard Model of particle physics (SM) the findings are combined. In the frame work of a gauge theory it describes the experimental data well, but there are still missing pieces. The Higgs boson discovery from 2012 is one of the more recent highlights because it confirmed an important part of the theory.

With the SM describing matter, there remain still open questions such as baryogenesis and neutrino masses, to name only two. Also, with the universe only consisting of about $5 \%$ visible matter, there needs to be more research in the dark matter and dark energy field which make up the rest of the universe. The Large Hadron Collider (LHC) at the European Organization for Nuclear Research (CERN) in Geneva provides a setup for research by colliding high energetic particles to create new particles. The measurements are conducted at the four interaction points by four experiments. ATLAS is one of the two multi purpose detectors.

In 2026 the LHC will be upgraded to the High Luminosity-LHC (HL-LHC) providing a higher instantaneous luminosity and a higher centre of mass collision energy. Consequently, the detectors have to be upgraded as well to be capable of handling the harsher radiation conditions and still perform high precision measurements. One part of the upgrade for the ATLAS experiment is the replacement of the inner tracking detector with a new, all silicon detector consisting of pixel and strip modules. Thousands of pixel modules are required to be built with high quality standards. Processes and tools to achieve such high quality are under development. In the following, studies of assembly tools are presented carried out with prototype modules. Once assembled, the modules have to be tested to prove their quality. For this purpose, a test stand prototype has been constructed. Its properties and qualifying tests are shown in this thesis.

Looking even more into the future with even higher particle fluences, silicon as sen- 


\section{Introduction}

sor material might not be feasible anymore. An alternative sensor material could be diamond. Synthetic polycrystalline diamond is cheaper compared to single crystals, but signal losses occur at the crystal boundaries. To circumvent this, the signal collecting electrodes can be implanted in the diamond itself using a femtosecond laser. Test beam measurements with such a 3D pixelated diamond detector and a planar pixelated diamond detector are conducted to obtain hit efficiencies and to study pixel geometry effects.

In Chapter 2 the SM is introduced and possible additions to it to motivate the continuation of research. As mentioned above, this work is carried out in the framework of the ATLAS experiment at the LHC. Their current state and the planned upgrades for both are described in Chapter 3. As the main topic of this thesis is particle detection, the interactions of particles with matter are explained in Chapter 4. A special focus is put on silicon and diamond as sensor materials. After these introductions, the research for module assembly procedures and module testing for the ATLAS upgrade is described in Chapter 5. The results of test beam measurements with diamond pixel detectors are displayed and discussed in Chapter 6. The last chapter, Chapter 7, concludes the work and gives an outlook on future developments of detector technologies. 


\section{CHAPTER 2}

\section{Motivation}

This work is conducted in the field of high energy particle physics (HEP). So the underlying theory of the field, the Standard Model of particle physics (SM), is introduced in the first section. Some limitations of it are discussed to motivate the continued research in the second section.

\subsection{The Standard Model}

Three fundamental forces are described in the SM, the electromagnetic, the weak, and the strong force. The bosons, particles with integer spin, mediate the forces between the particles. The second group of particles are the fermions with half integer spin, see a graphical representation in Figure 2.1, the quarks and the leptons. Theses two groups are distinguished based on the charges carried by the particles. The quarks are the only particles to interact with the strong force, because they have a colour charge, which allows the coupling to the gluons $g$, the mediators of the strong force. The leptons do not interact with the strong force.

Both groups are organised in three pairs, called generations, of weak isospin partners, charged lepton and corresponding neutrino, and up-type and down-type quark. The quantum mechanical properties of the pairs are the same, only the masses of the particles increase with increasing generation. Because of this, only the pair with the smallest masses is stable, the other particles decay over time. These decays are described with the weak force, which is mediated by three bosons, an electrically neutral $Z^{0}$, and two electrically charged $W^{ \pm}$bosons.

The photon $\gamma$, the mediator of the electromagnetic force, couples to all electrically charged particles. It has no charge and also no rest mass. All fermions, except the neutrinos, carry an electrical charge; integer elementary charges for the remaining leptons, and one third and two thirds charges for down- and up-type quarks, respectively. 


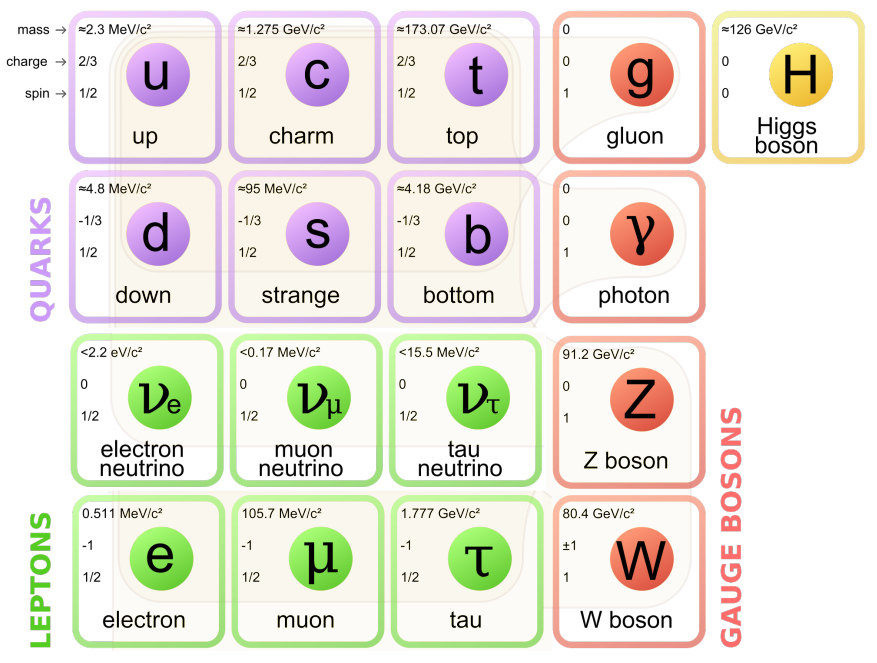

Figure 2.1.: Particles and their properties contained in the SM. Image credit Wikimedia Commons.

With the Higgs mechanism and the Yukawa coupling, the mass generation of the weak bosons and the fermions is explained. The coupling is mediated by the Higgs boson, predicted in $1964[1,2]$, and recently discovered by the ATLAS and CMS experiments at CERN $[3,4]$. The neutrinos are treated as massless in the SM, which they are not as experiments observing neutrino oscillation discovered, see next section [5].

Interactions between the bosons themselves are possible, because some of them carry also charges of the other forces or their own charge. In this way triple or quartic gauge boson couplings are realised as rare processes in the SM e.g. $W^{+} W^{-} Z^{0}$ and gluon self coupling but not interactions of $Z^{0} \mathrm{~s}$ and $\gamma \mathrm{s}$.

It is to mention that for every fermion an anti-particle exists with the opposite electrical charge, but otherwise the same properties. This concludes the particle content of the SM. Its greatest feature is the predictive power from large cross section processes to very rare interactions. In Figure 2.2, the results from the ATLAS experiment are compared to the SM for the wide range of measurements that have been conducted. At the moment, these results, and the results of other experiments, agree with the expectations of the SM. But there are also phenomena observed that are not included in the SM, as of now.

\subsection{Beyond the Standard Model}

The SM aims at describing all particles in the universe and their interaction. In astronomical observations, the rotational speed of galaxies has been found to deviate from the expectation calculated from the visible content of the galaxy [7-9]. This extra mass needed to explain the measurements is called dark matter. There is about five times the amount of dark matter compared to the visible matter in the universe, and up to now 


\section{Standard Model Production Cross Section Measurements}

Status: November 2019

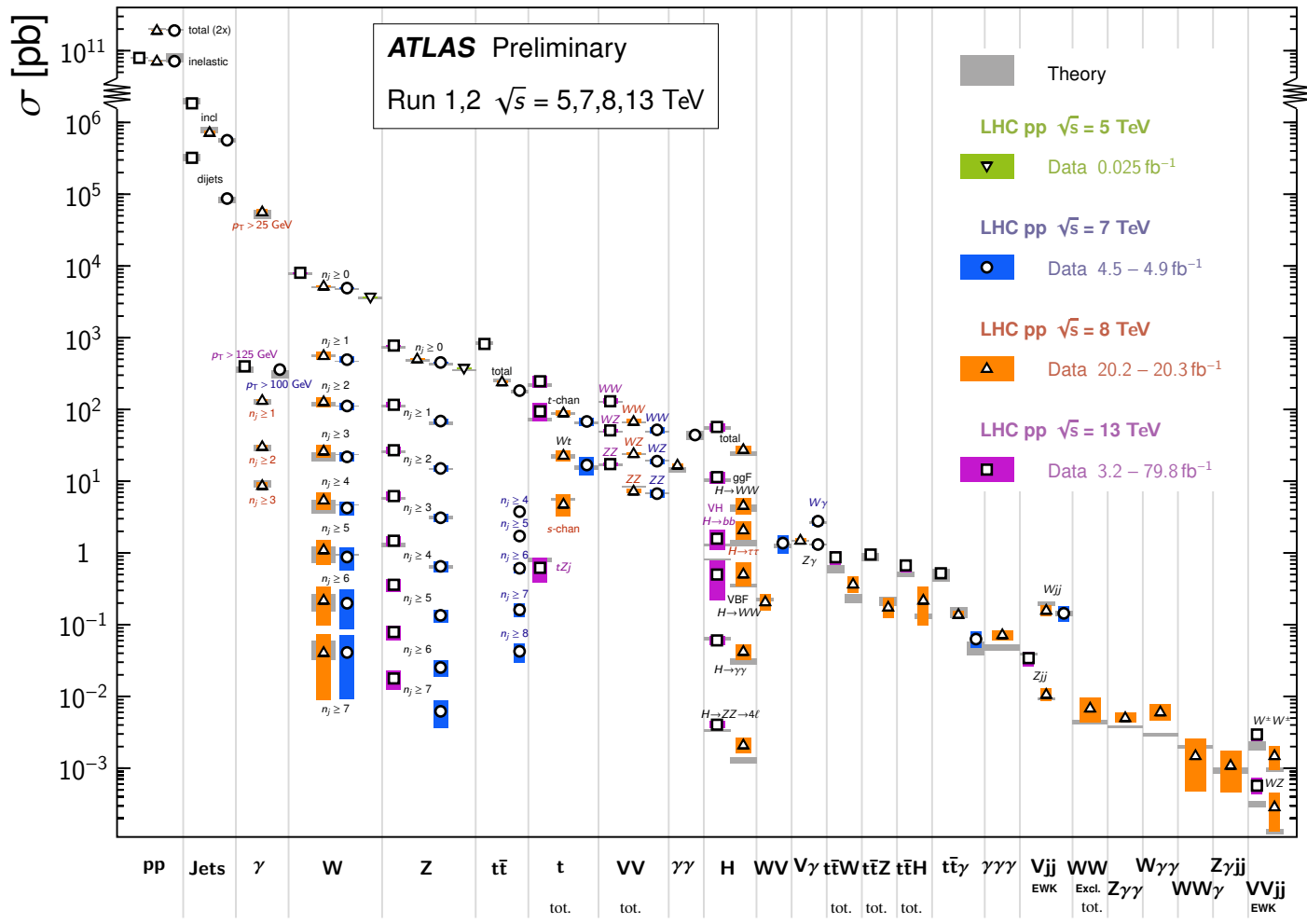

Figure 2.2.: Measurements conducted by the ATLAS experiment compared to the SM predictions [6].

no particle candidate has been found that could be associated to it [10].

Beyond the SM theories (BSM), such as super-symmetry (SUSY), require new particles, in this case a so-called super partner for every standard particle, which is a fermion for a boson partner and vice versa. Because no such particles have been observed so far, the symmetry has to be broken and the super partners are presumably more massive. If also the super particles can only be produced in pairs from standard particles, the lightest super particle would be stable and a good candidate for dark matter or more specific for a weakly interacting particle (WIMP). Such particles could be detected in the production by missing energy in the system or in the decay by displaced vertices, because the particles travel a distance before decaying.

As mentioned above, neutrinos are massless in the SM. One major source of neutrinos is the fission process in the sun, which produces electron neutrinos. With the model of the sun, the neutrino flux expected on earth can be calculated. The measurements show a deficit of electron neutrinos, which was resolved by the Super-Kamiokande experiment, observing muon neutrinos of the same rate [5]. This effect is explained with oscillations between neutrino flavours, that are only possible if the neutrinos have a non-zero mass. 


\section{Motivation}

The generation of the neutrino mass is, however, not included in the SM.

One very obvious observation is that the universe consists of matter. This raises the question where or how the anti-matter vanished if it was produced in pairs with matter in the early stages of the universe. Something needs to create an asymmetry between matter and anti-matter. CP-violation found in the SM can be a part, but not the sole reason to explain the current state.

Searches for new physics happen at two fronts: at higher energies, to boost the production of more massive particles and in higher precision measurements, to find deviations from the SM induced by corrections from higher energy scales. For the latter one, measurements with the Higgs boson or the top quark, the heaviest particle known, are good test benches, because of the important role in the SM and the high mass. The main decay for both involves the second heaviest quark, the $\mathrm{b}$ quark. It has a rather long lifetime so that it can travel a short distance from the creation point before decaying. With a good track reconstruction the decay products can be traced back and the b quark can be identified as such due to the different origin of the track.

At the future collider experiments like the HL-LHC, many interactions will happen at once producing lots of particle tracks. To ensure that also in this difficult environment high precision measurements are possible, new techniques and materials have to be developed and studied to build suitable detectors. 
CHAPTER 3

\section{LHC and ATLAS Detector}

Tests of the SM are conducted in many research facilities and laboratories. As the ATLAS experiment represents the target of this work, the Large Hadron Collider, located at CERN, is introduced in Section 3.1 and the ATLAS detector in Section 3.2. After that the upcoming upgrades of both are described in Section 3.3.

\subsection{Large Hadron Collider}

The Large Hadron Collider (LHC) is a circular collider for mainly protons with the option for heavy ions [11]. It is located around Geneva about $100 \mathrm{~m}$ underground and operated by CERN. In the $27 \mathrm{~km}$ long ring, the protons are accelerated to their final energy of up to $7 \mathrm{TeV}$. The acceleration is achieved with RF-cavities, generating a bunch structure in the beams. The protons are guided around the circle by super conducting dipole magnets with a magnetic field strength of $8.33 \mathrm{~T}$, which are constructed such that both beams running in opposite directions are accommodated next to each other.

Before the protons can be injected in the LHC, they have to be pre-accelerated to match the magnets operation range. The full accelerator chain present at CERN is shown in Figure 3.1. The journey of the protons starts at the linear accelerator LINAC 2 where they reach an energy of $50 \mathrm{MeV}$. Then a chain of circular accelerators follows with the Proton Synchrotron Booster (PSB or BOOSTER), the Proton Synchrotron (PS), and finally the Super Proton Synchrotron (SPS), gradually increasing the energy of the beam to $1.4 \mathrm{GeV}, 25 \mathrm{GeV}$ and $450 \mathrm{GeV}$ before passing it into the LHC.

The beams are brought to collision at four interaction points (IP) throughout the ring by focussing quadrupole magnets. Around these points the four experiments ALICE, ATLAS, CMS and LHCb have built their detector [12-15]. The detectors of ATLAS and CMS are multi purpose detectors, while LHCb focusses on b quark physics and ALICE on heavy ion interactions. 


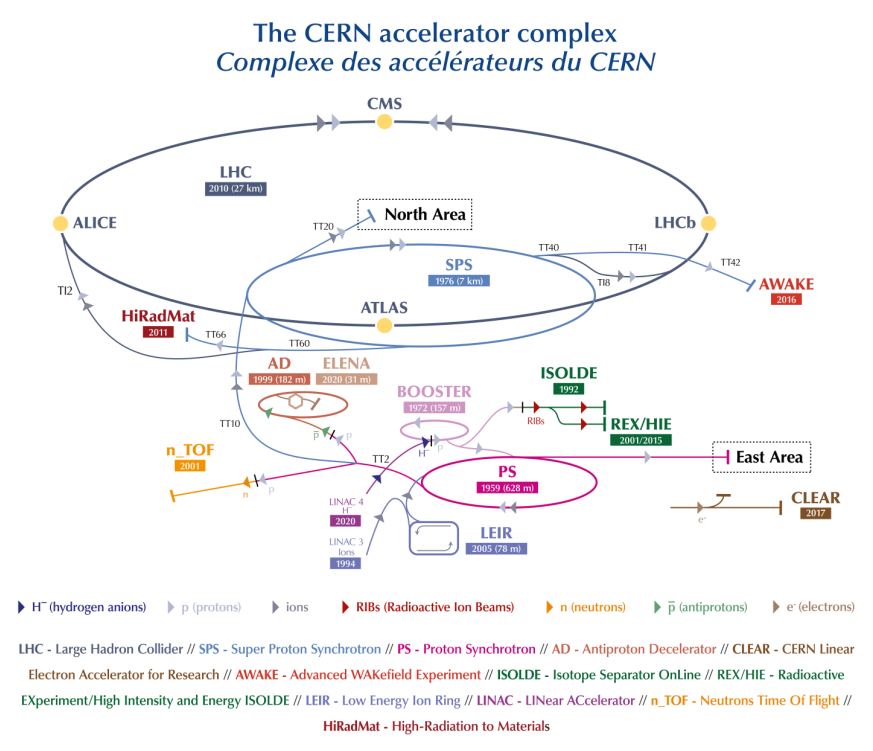

Figure 3.1.: The accelerator complex at CERN. (C) 2019 CERN.

\subsection{ATLAS Detector}

The ALTAS detector is installed at the IP 1 in the LHC. It has a length of $44 \mathrm{~m}$, a height of $25 \mathrm{~m}$ and weighs $7500 \mathrm{t}$. A schematic drawing in Figure 3.2 shows the layered structure to measure as many types of particles as possible and their properties produced in the proton interactions in the centre of the detector.

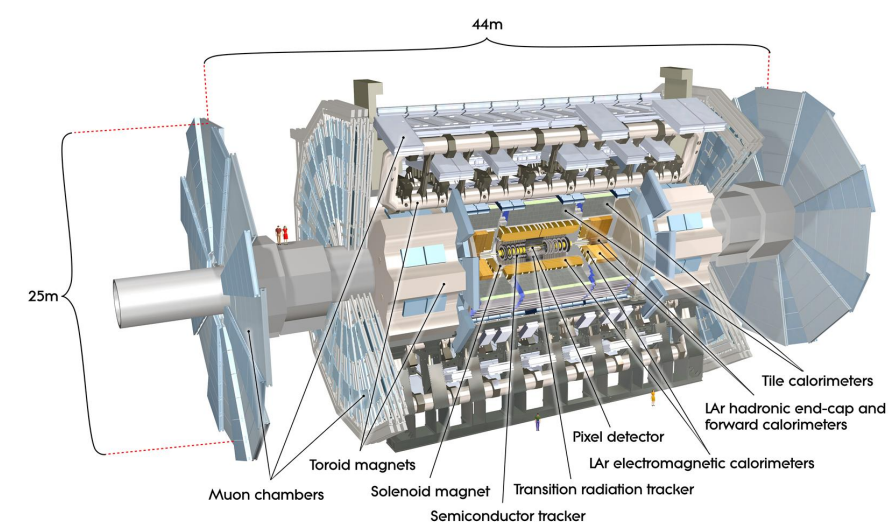

Figure 3.2.: Schematic of the ATLAS detector. ATLAS Experiment (C) 2008 CERN.

The centre of the interactions is the origin of the coordinate system in ATLAS with the $z$-axis pointing in the direction of the beam, the $x$-axis pointing towards the centre 
of the accelerator ring, and the $y$-axis pointing upwards, completing the right-handed coordinate system. Two angles are defined: $\phi$ around the $z$-axis as azimuthal angle, and $\theta$ as polar angle from the $z$-axis. The pseudo-rapidity is defined as $\eta=-\ln \tan (\theta / 2)$.

\subsubsection{The Inner Detector}

The innermost part of the ATLAS detector is the inner detector (ID), a tracking detector system for charged particles. It is immersed in a $2 \mathrm{~T}$ solenoid magnetic field to bend the trajectory of the particles and thus gain information about the sign of their electrical charge.

From the innermost part to the outermost, there are the insertable B-layer (IBL), the Pixel detector (Pixel), the silicon microstrip layers (SCT), and the transition radiation tracker (TRT).

\section{The Pixel Detector}

In Figure 3.3, the Pixel detector with its support structure is shown. It consists of three barrel layers and three endcap discs at each side of the barrel section. The coverage extends to $|\eta|=2.5$.

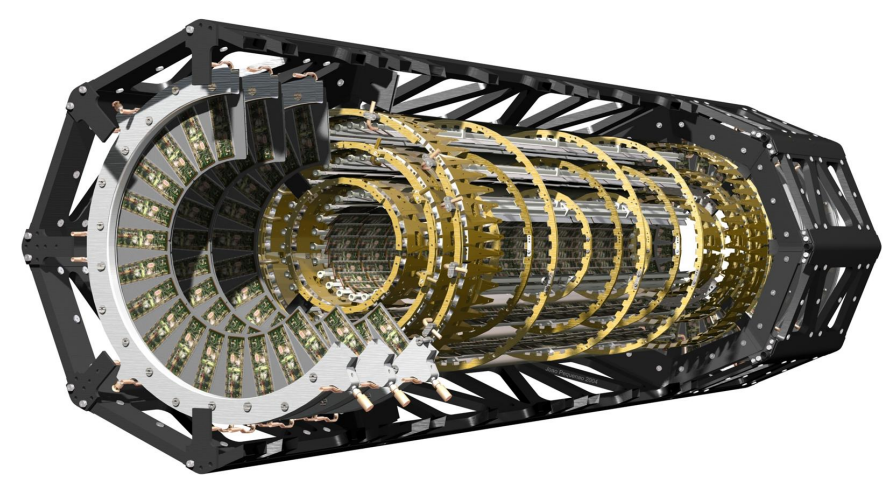

Figure 3.3.: The ATLAS Pixel detector before installation of the IBL. ATLAS Experiment (c) 2008 CERN.

The detector modules are hybrid pixelated silicon modules, consisting of an $n^{+}$-in- $n$ sensor and the FE-I3 read-out chip. The sensor is designed such that even after type inversion of the n-type bulk due to radiation damage the full sensor can be depleted to collect the maximum amount of charge. To counter radiation damage induced effects like increased leakage current and changes to the doping concentration due to diffusion and reverse annealing, the detector is kept at low temperatures. More about detector design can be found in Chapter 4.

The sensor is divided into pixels with a pitch of $400 \mu \mathrm{m} \times 50 \mu \mathrm{m}$, each connected pixel by pixel to the read-out chip. The thickness of the sensor is $256 \mu \mathrm{m}$. 


\section{3. $L H C$ and ATLAS Detector}

\section{The Insertable B-Layer}

In the first long shut down period of the LHC, an additional pixel layer, the insertable B-Layer, was added between the IP and the first pixel layer [16]. The radius of the beam pipe had to be reduced to fit the new layer.

With this new layer, an additional measurement point for the tracks closer to the IP increases the tracking performance. It also helps with covering deficiencies in the other pixel layers, mostly the previous first layer, due to radiation damage. Not only the radiation damage but also the particle rate posed difficulties for the Pixel detector, because the LHC performed better than expected, delivering more interactions per bunch crossing. Introducing the IBL reduces the impact of bandwidth limitations from the other layers on the tracking performance.

The IBL serves also as a test bench for new technologies on the way to the high luminosity phase of the LHC. Because of the spatial limitations in the detector, the active sensor area has to be maximised. This lead to developments of slim edges, reducing the inactive distance to the edge from $1100 \mu \mathrm{m}$ in the Pixel detector to $200 \mu \mathrm{m}$ in the IBL.

Two types of sensors are used for the IBL: one with planar pixel geometry, a $n^{+}$-in- $n$ type sensor as used in the Pixel detector, but only $200 \mu \mathrm{m}$ thick, and a sensor with 3D electrode geometry in the bulk of the sensor with a thickness of $230 \mu \mathrm{m}$. The later one features an intrinsic higher radiation hardness, because the close spacing of the electrodes allows to reduce the bias voltage and thus the leakage current, and counters charge trapping.

Both sensors are connected to the FE-I4 read-out chip, which has a pixel pitch of $250 \mu \mathrm{m} \times 50 \mu \mathrm{m}$. More details can be found in Section 4.4.

\section{The Silicon Strip Detector and the Transition Radiation Tracker}

Further out in radius, after the Pixel detector, the silicon strip layers are placed. They consist of four barrel layers and nine endcap disks at each side. Two layers of strips with a pitch of $80 \mu \mathrm{m}$ are used per module, slightly rotated against each other to provide full two dimensional position resolution.

The TRT consists of $4 \mathrm{~mm}$ diameter drift tubes with a wire in the middle that is directly read out. They are filled with a $\mathrm{Xe}, \mathrm{CO}_{2}, \mathrm{O}_{2}$ gas mixture. Particles produce transition radiation at the polypropylene fibres between the drift tubes. Because the radiation depends on the relativistic $\gamma$ factor, electrons can be distinguished from other passing particles with the TRT. Depending on the position in the detector, up to 36 measurement points for a particle trajectory are added by the TRT.

\subsubsection{Calorimeter}

The calorimeters are located outside of the solenoid and provide the energy measurement of the particles by stopping them in the material and recording the deposited energy. The calorimeter system of ATLAS is divided in two conceptual parts: the electromagnetic calorimeter and the hadronic calorimeter. The first one measures the energy of electrons 
and photons and provides resolution for the determination of the origin of the photons, either from the main interaction or only from secondary particles, mainly $\pi^{0}$. The second one can measure all hadronic jets without spilling out particles into the outer systems.

The electromagnetic calorimeter consists of lead absorber plates and liquid argon (LAr) as active material. They are arranged in an accordion shape to provide radial segmentation. Divided in a barrel and an endcap part, it covers a pseudo-rapidity range of $|\eta|<3.2$.

The hadronic calorimeter is divided in three parts, the Tile calorimeter, the HadronicEndcap-Calorimeter (HEC) and the Forward Calorimeter (FCal). The Tile calorimeter is located in the barrel region further out from the electromagnetic calorimeter and covers up to $|\eta|<1.7$. It is made of steel absorber and scintillators as active material. The light is collected with wavelength shifting fibres and recorded by photomultipliers. The HEC is placed behind the endcaps of the electromagnetic calorimeter with a coverage of $1.5<|\eta|<3.2$. It consist of LAr and copper plates as absorbers. Most forward, covering the range of $3.1<|\eta|<4.9$, the FCal is installed. LAr is also used as active material and copper and tungsten as absorbers to optimise for electromagnetic and hadronic interactions, respectively.

\subsubsection{Muon Chambers}

Outside of the calorimeter system, the only detectable particles are muons, because, as minimum ionising particles, they are not stopped in the calorimeters. In the muon chambers their trajectory is bent again with a toroidal magnetic field, and tracked with a range of multi wire detectors. As muons are produced in many interesting physics processes, the signal from the muon chambers is used as a fast trigger for the selection of events.

Monitored Drift Tubes (MDT) and Cathode Strip Chambers (CSC) are used for tracking the muons in a range up to $|\eta|<2.7$. The CSC are placed closer to the IP, because they have a higher counting rate of $1000 \mathrm{~Hz} / \mathrm{cm}^{2}$, compared to the rate of MDTs of $150 \mathrm{~Hz} / \mathrm{cm}^{2}$. For the fast trigger signal Resistive Plate Chambers (RPC) are used in the barrel region, and Thin Gap Chambers (TGC) in the endcaps, covering together the range up to $|\eta|<2.4$.

\subsection{HL-LHC and ATLAS Upgrade}

As discussed in Chapter 2, there are still many open questions in the field of particles physics. To provide the data for more precise measurements, the LHC will be upgraded to the High Luminosity-LHC. 


\section{3. $L H C$ and ATLAS Detector}

\subsubsection{HL-LHC}

The instantaneous luminosity $L_{\text {inst }}$ relates the event rate $\mathrm{d} N / \mathrm{d} t$ to the cross section $\sigma$ of a process:

$$
\frac{\mathrm{d} N}{\mathrm{~d} t}=L_{\text {inst }} \times \sigma .
$$

It can also be expressed in parameters of the accelerator:

$$
L_{\text {inst }}=\frac{n_{\mathrm{b}} N^{2} f_{\mathrm{rev}}}{4 \pi \beta^{*} \epsilon_{\mathrm{n}}} R \text {. }
$$

With the number of bunches in the machine $n_{\mathrm{b}}$, the number of protons per beam $N$, the revolution frequency $f_{\text {rev }}$, the beam beta function $\beta^{*}$, the transverse normalised emittance $\epsilon_{\mathrm{n}}$, and a geometrical reduction factor composed of the beam crossing angle $\theta_{\mathrm{c}}$, RMS bunch length $\sigma_{\mathrm{z}}$, and RMS beam size $\sigma^{*}: R=1 / \sqrt{1+\frac{\theta_{\mathrm{c}} \sigma_{\mathrm{z}}}{\sigma^{*}}}$. For the HL-LHC upgrade, most of these parameters can and will be improved to deliver more data to the experiments [17].

Only right at the start, the beam intensity and brightness can be controlled, because the phase space is conserved according to the Liouville theorem. One of the main changes is the replacement of the LINAC 2 with the LINAC 4, which is anticipated to provide a doubled beam brightness at the start of the accelerator chain $[18,19]$.

To improve $\beta^{*}$, the beam focus spot is reduced with stronger quadrupole magnets at the interaction point. Because the beam angle has to increase for that, additionally crab cavities are installed to tilt the bunches at the interaction point in a more favourable angle to increase the geometric overlap of the beams. More interactions per bunch crossing are the result, which increases the instantaneous luminosity. Up to a mean number of 200 of such pile up events are anticipated, posing a challenge for the detectors because of the large track density and occupancy in the innermost layers. To mitigate this effect for the detectors, not the maximum possible instantaneous luminosity will be provided at the start of a measurement run, but a reduced one. This way, the instantaneous luminosity is kept on a high level for a longer period of time for the run, delivering data in a controlled rate, because the decrease over time due to the interaction of particles and other losses can be compensated by improving the beam parameters.

The roadmap to the HL-LHC is shown in Figure 3.4. In the long shut down 3 (LS3) the machine and detector upgrades will be installed. In the current scenario, a data set of an integrated luminosity of $4000 \mathrm{fb}^{-1}$ will be collected by each experiment up until the end of the run period in 2040 .

\subsubsection{ATLAS Upgrade}

At the end of run 3, before the LS3, the ID of ATLAS will be at the end of its lifetime. It will be replaced by the inner tracker (ITk), which is designed to withstand the harsh radiation conditions at the HL-LHC ${ }^{1}$.

\footnotetext{
${ }^{1}$ This section follows and cites in parts a previous publication of the author of this thesis [20]
} 


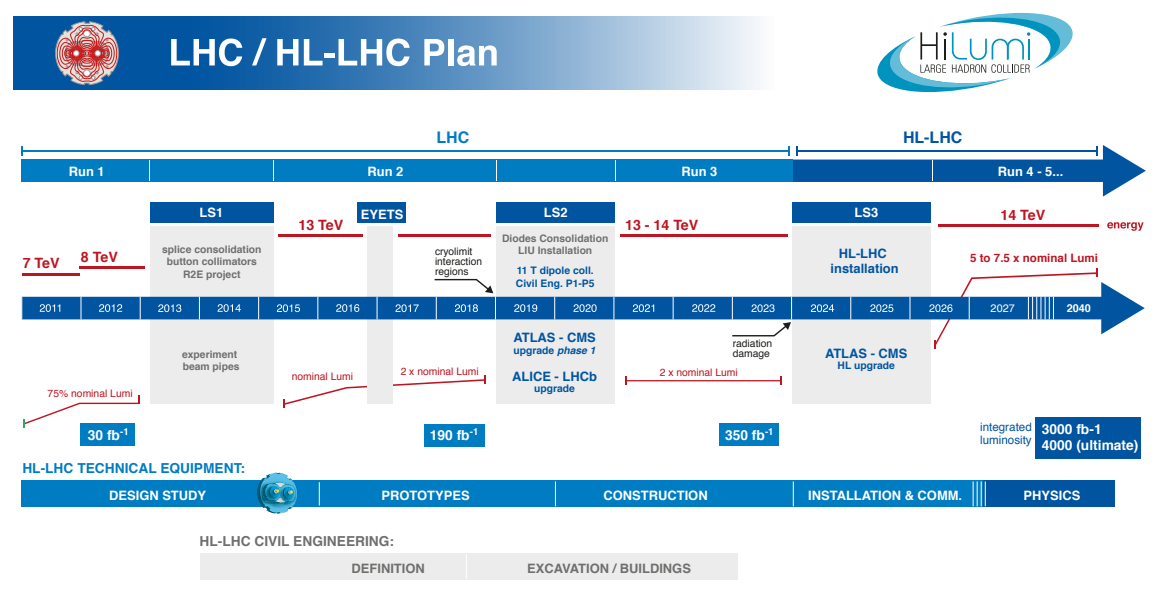

Figure 3.4.: Time line of the LHC and the following HL-LHC phase. CCCERN

\section{ATLAS ITk}

In Figure 3.5(a), the layout of the active components of the ITk is displayed. It is an all silicon based detector with five layers of pixel modules closest to the IP in the barrel region, and four strip module layers further outside. Multiple rings in the endcaps extend the coverage to $|\eta| \leq 4$. A full description can be found in the ATLAS ITk Pixel TDR [21]. A selection of features are compared to the current ID in Table 3.1. In the following, the focus will be on the pixel detector, as the work presented here is targeted at this part.

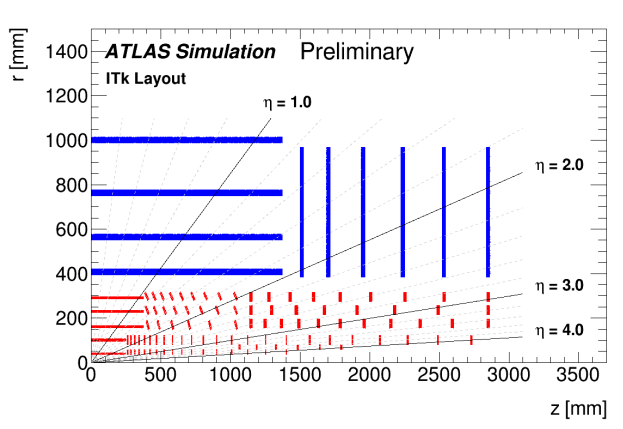

(a)

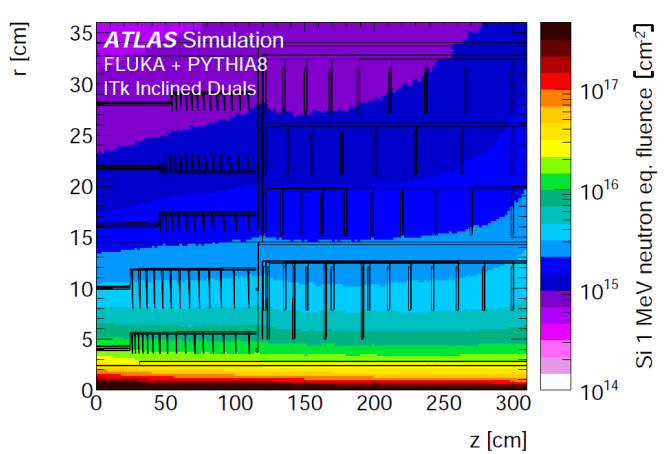

(b)

Figure 3.5.: (a): schematic layout of one quadrant of the ATLAS ITk in beam direction $z$ and radial direction $r$ with silicon strip modules in blue and pixel modules in red [22]. (b): $1 \mathrm{MeV}$ neutron equivalent fluence in the pixel detector normalised to $4000 \mathrm{fb}^{-1}[21]$. 


\section{3. $L H C$ and ATLAS Detector}

\begin{tabular}{|l|c|c|}
\hline & LHC Run2 ID & HL-LHC ITk \\
\hline Si area Pixel $\left[\mathrm{m}^{2}\right]$ & 1.9 & 13 \\
\hline Si area Strips $\left[\mathrm{m}^{2}\right]$ & 60 & 160 \\
\hline Channels Pixel & $\approx 9.2 \times 10^{6}$ & $\approx 5 \times 10^{9}$ \\
\hline Channels Strips & $\approx 6 \times 10^{6}$ & $\approx 50 \times 10^{6}$ \\
\hline$|\eta|$ coverage & 2.5 & 4.0 \\
\hline Pixel size $\left[\mu \mathrm{m}^{2}\right]$ & $50 \times 400$ and $50 \times 250$ & $50 \times 50$ or $25 \times 100$ \\
\hline L1 Trigger rate $[\mathrm{kHz}]$ & 100 & 1000 \\
\hline
\end{tabular}

Table 3.1.: Comparison of the ATLAS ID with the ITk.

To be able to provide a high precision tracking performance under HL-LHC conditions, three main topics are improved compared to the ID: granularity, read-out speed and radiation tolerance. A higher granularity is needed to keep the occupancy per pixel low and to be able to distinguish tracks. This also reduces the bandwidth needed to read out the modules. Sensor pixel sizes of $(50 \times 50) \mu \mathrm{m}^{2}$ or $(25 \times 100) \mu \mathrm{m}^{2}$ are planned, and show in simulation an occupancy per channel of $0.16 \%$ in the innermost layer.

An increased granularity leads to more channels to be read out. Within the RD53 project [23] at CERN, ATLAS and CMS experiments have developed a new read-out chip, also known as RD53A [24]. It has a pixel size of $50 \times 50 \mu \mathrm{m}^{2}$ and a readout speed of up to $4 \times 1.28 \mathrm{~Gb} / \mathrm{s}$. The radiation tolerance exceeds $500 \mathrm{Mrad}$. To counter the effects of radiation induced bit flips, it is possible to reload the chip configuration during data taking. The read-out chips will be connected in serial powering chains in the detector to reduce the amount of cabling needed, which takes up space and deteriorates the tracking performance by multiple scattering. All pixel layers will use the same read-out chip that will be an ATLAS specific derivation of the RD53A chip. Towards the end of 2019, ATLAS will submit a new version of the chip named ITKpixV1.

In Figure 3.5(b), the neutron equivalent fluence in the pixel detector is displayed for the full runtime $\left(4000 \mathrm{fb}^{-1}\right)$. Different pixel sensor technologies are used in the different layers to cope with the radiation damage. In the innermost layer, 3D sensors of $150 \mu \mathrm{m}$ active thickness are used; for the other layers, planar sensors with $100 \mu \mathrm{m}(150 \mu \mathrm{m})$ thickness are expected in the second (third and fourth) layer. Because the radiation damage is too high for the readout chip in the two inner layers, it is foreseen that this region will be replaced after half of the runtime, hence accumulating only the fluence of $13 \times 10^{15} \mathrm{n}_{\mathrm{eq}} / \mathrm{cm}^{2}$. To cope with the high level of leakage current after radiation, the pixel detector will be cooled to $-25^{\circ} \mathrm{C}$ via $\mathrm{CO}_{2}$ cooling.

Because the complete ID will be replaced, the area to be instrumented with pixel modules increases to $13 \mathrm{~m}^{2}$. A total of about 12000 modules have to be built for the pixel detector. To reduce the cost per module, n-in-p sensors are the considered technology, because they are produced in a single sided process, which makes it cheaper. Also, the sensors are produced in a size to connect four read-out chips to reduce the amount of individual pieces.

Not only the cost has to be considered, but also the time it takes to produce and 
install the modules in the detector. Based on the production of the IBL, tools are under development to facilitate the precise and timely production of the modules. First result will be presented in Chapter 5 .

\section{Outer Barrel Demonstrator Project}

Developing and producing high quality components for the ITk is only the start. These parts have to be functioning also in combination integrated into the sub systems of the ITk. Testing larger scale structures is done in the so-called demonstrator projects. Many different ones are conducted at the moment, from read-out chains of multiple modules to e.g. ring structures for the endcaps. The work presented here is part of the FE-I4 outer barrel stave demonstrator project [25]. The main goals of the project are building a stave and loading it with modules, developing the read-out of a many module system, testing of $\mathrm{CO}_{2}$ cooling, and setting up a Detector Control System (DCS).

The demonstrator is located in an ATLAS laboratory at CERN. The main part, the stave with the modules, is housed in a box to shield it from external influences like light sources, and to achieve a controlled environment, see Figure 3.6(b). A cooling plant is available for cooling the stave with $\mathrm{CO}_{2}$.

A schematic of the stave can be found in Figure 3.6(a). It is made of light weight carbon fibre composites and the cooling pipes are integrated. The electrical wires for bias voltage and supply current for the modules are routed on the side. The modules are loaded on top of the stave with quad chip modules flat in the centre of the stave, and double chip modules inclined towards the sides of the stave. In total, there are 120 FE, organized in 14 quad chip modules and 32 double chip modules. They are grouped in serial powering chains to reduce the material budget for cabling.

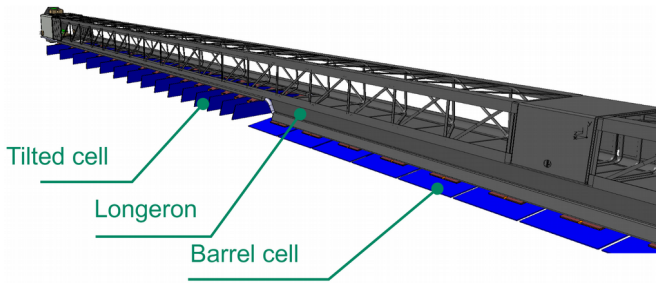

(a)

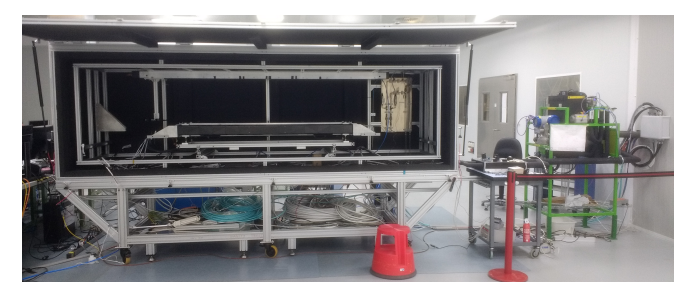

(b)

Figure 3.6.: (a): schematic of the demonstrator stave. (b): the setup in the CERN laboratory ${ }^{2}$.

The electrical read-out links from the chips are aggregated with GBTx chips to optical links, which are then connected to the read-out system [26]. Because of the $160 \mathrm{Mbit} / \mathrm{s}$ bandwidth per electrical link, 20 channels can be combined with one GBTx chip, requiring 6 GBTx chips for the full stave [27]. Different read-out systems are tested, e.g. the

\footnotetext{
${ }^{2}$ Courtesy from Eric Buschmann
} 


\section{3. $L H C$ and ATLAS Detector}

RCE and Yarr systems to be able to compare the results and improve the systems on the way to the ITk.

A DCS is under development for the demonstrator, consisting of an interlock and control system [28]. The control system monitors key parameters such as temperatures and voltages. The interlock system protects the detectors by checking the environment of the box, and can turn off power supplies for the serial powering chains in cases of emergency. 


\section{CHAPTER 4}

\section{Solid State Pixel Detectors}

In this chapter ${ }^{1}$, basic interactions of particles with matter are discussed with a focus on charged particles and photons based on [30]. From the theory to the application, silicon and diamond as sensor materials are discussed. The detectors of interest in this work are hybrid detectors, which are introduced in the last section of this chapter.

\subsection{Particle Detection with Solid State Detectors}

The understanding of the interaction of particles with matter is the key point for designing a particle detector. Different materials have varying responses to particles passing through, different particles interact differently, even the same particles with higher or lower energy behave differently. Particle detection relies on these interactions, but not all effects are non destructive, e.g. the material can get altered by the passage of a particle, resulting in so-called radiation damage.

\subsubsection{Cross section}

The cross section describes the probability of an interaction to take place. If a beam of particles hits a target, either another beam or a block of material, one can define the differential cross section $\mathrm{d} \sigma / \mathrm{d} \Omega$ as the average number of particles scattered in the angle $d \Omega$ per unit time and per unit particle flux. The total cross section can be derived by integration over the angle $d \Omega$. In general, this will depend on the particle type and energy.

\footnotetext{
${ }^{1}$ This chapter is based on and cited from the master thesis of the author of this thesis [29].
} 


\section{Solid State Pixel Detectors}

\subsubsection{Charged particles}

The dominant force for interactions of charged particles with matter is the electromagnetic force. The particles scatter with the electrons or the nucleus via the Coulomb potential. Basically, particles excite or ionize atoms on their way and hence lose energy and get deflected from the original path. Most theories describing the interaction assume the electrons of the atoms to behave as free, because the energy and the mass of the particles are much higher than the binding energy of the electron. If the particle itself is an electron other models have to be used, same for very heavy ions. The focus here is on heavy charged particles in the range of muons, protons and $\alpha$-particles.

The mean energy loss, or stopping power per path length $\left\langle\frac{\mathrm{d} E}{\mathrm{~d} x}\right\rangle$ for such particles can be calculated with the Bethe-Bloch-formula:

$$
\left\langle\frac{\mathrm{d} E}{\mathrm{~d} x}\right\rangle=-4 N_{A} r_{e}^{2} m_{e} c^{2} z^{2} \rho \frac{Z}{A} \frac{1}{\beta^{2}}\left[\frac{1}{2} \ln \left(\frac{2 m_{e} c^{2} \gamma^{2} \beta^{2}}{I^{2}} T_{\max }\right)-\beta^{2}-\frac{\delta}{2}-\frac{C}{Z}\right] .
$$

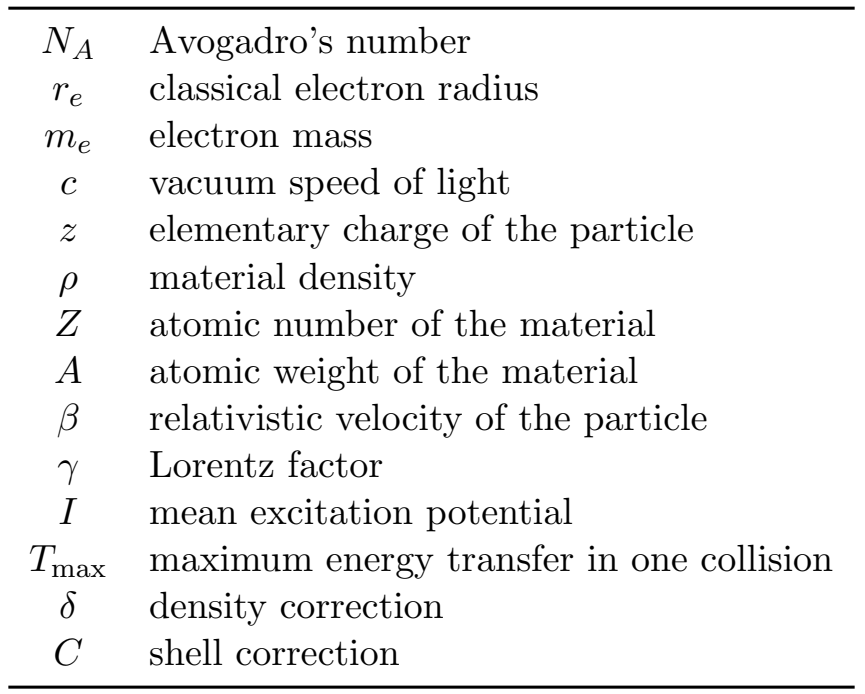

Table 4.1.: Used variables in equation (4.1).

The plot belonging to the formula can be found in Figure 4.1 for muons in copper, and the variables in it are listed in Table 4.1. In the following, the individual parts are explained.

The maximum energy transfer $T_{\max }$ is achieved by a head on collision and the result of the kinematics with a particle of mass $M$ is:

$$
T_{\max }=\frac{2 m_{e}(c \beta \gamma)^{2}}{1+2 m_{e} / M \sqrt{1+(\beta \gamma)^{2}}+\left(m_{e} / M\right)^{2}} .
$$

The mean excitation potential $I$ is an average of the atomic levels. Since this is difficult to calculate, empirical formulas are used, but they do not display variations at closing 


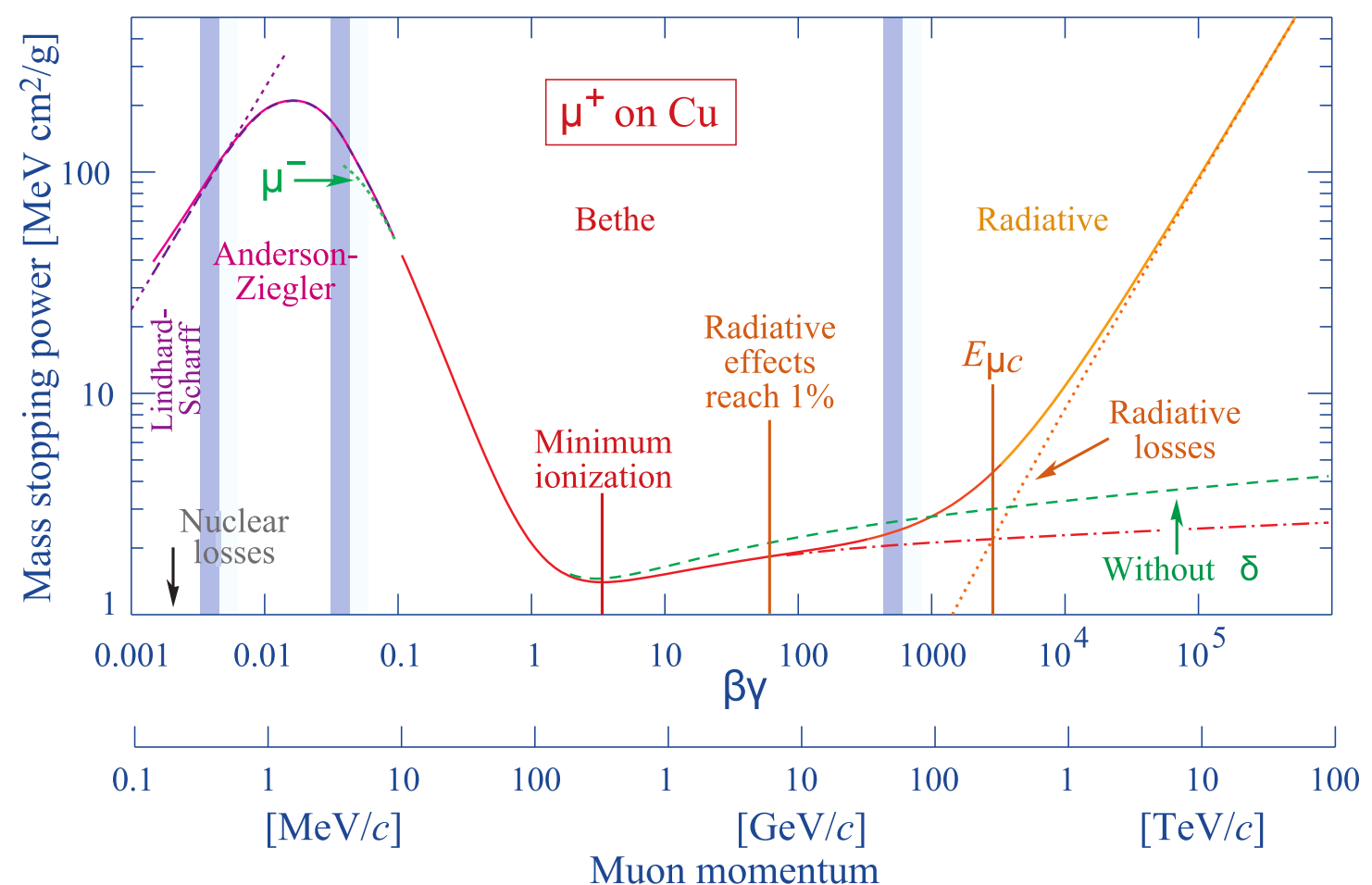

Figure 4.1.: Stopping power over a wide range of particle momenta and energy for muons in copper [31].

atomic shells:

$$
\begin{array}{ll}
I=12 Z+7 \mathrm{eV} & Z<13 \\
I=9.67 Z+58.8 Z^{-0.19} \mathrm{eV} & Z \geq 13 .
\end{array}
$$

The density correction $\delta$ accounts for the polarization of the atoms along its path. Because of this, far away electrons are shielded from the field of the particle and add less to the energy loss. With higher particle velocity, the influence of electrons further away rises, making this effect important for high energetic particles. The higher the density of the material, the higher the polarization, and hence the name density correction.

The shell correction is a small contribution for low energies when the particle and electrons have comparable velocities, and the electron thus cannot be approximated as stationary. In this region, the Bethe-Bloch formula breaks down.

For low particle energies, the $\beta^{-2}$ part is dominant. Different particles can be separated in this region with their specific energy loss, because the slope is shifted for different masses. At a $\beta \gamma=3-4$, particles reach a minimum energy loss and are therefore called minimum ionizing particles (m.i.p.). This loss is of about $2-3 \mathrm{MeV}$. With higher momentum the loss increases again slightly due to relativistic effects and reaches a plateau. 


\section{Solid State Pixel Detectors}

The energy loss of a particle in a material depends on the energy of the particle. A high energetic particle will lose only little energy at the beginning and the rate increases drastically after it is no more a m.i.p. and reaches the $\beta^{-2}$ part. At this point, the particle gets stopped in the material within a short distance. Most energy is deposited near the stopping location. This behaviour is described by the Bragg-curve.

For thinner detectors, the probability distribution of the energy loss can be described by the Landau distribution [32-34]. It is a very asymmetric distribution, because only rarely high energy transfer collisions happen. Most of the time small energies are transferred. The mean energy loss is dominated by the high weights and therefore shifted towards the tail of the distribution. A more suitable parameter to describe the behaviour is the most probable energy loss.

\subsubsection{Multiple scattering}

Charged particles can also collide elastically via the Coulomb potential with the nuclei instead of the electrons. This can be described with the Rutherford formula:

$$
\frac{\mathrm{d} \sigma}{\mathrm{d} \Omega}=z_{2}^{2} z_{1}^{2} r_{e}^{2} \frac{\left(m_{e} c / \beta p\right)^{2}}{4 \sin ^{4}(\theta / 2)} .
$$

Most collisions will have a small scattering angle $\theta$ due to the $\sin ^{-4}(\theta / 2)$ dependence. Many small deflections of the particle lead to a zigzag path and an overall scattering angle can be defined. Single scattering in very thin materials follows the Rutherford formula. A medium number of interactions cannot be easily described, but for many scatterings, statistical methods can be applied. To discuss these is not aim of this thesis. Further information can be found in [30].

\subsubsection{Photons}

Since photons are electrically neutral, they do not scatter with the electrons of the atoms as charged particles do. The main interactions in material for photons are the photoelectric effect, Compton scattering and pair production. The first and last mentioned processes remove the interacting photon completely. The Compton scattering changes its energy. The intensity $I$ of a beam of photons exponentially decreases during the passage through the material from the starting intensity $I_{0}$ with an attenuation coefficient $\kappa$ :

$$
I(x)=I_{0} \exp (-\kappa x)
$$

\section{Photoelectric effect}

The absorption of a photon by an atomic electron and emission of that electron is known as the photoelectric effect. The electron energy is then the photon energy minus the binding energy. The electron has to be bound so the nucleus can take the recoil momentum for conservation of momentum. 
The cross section for this process is high for low photon energies, because the higher shell electrons have less binding energy. It decreases fast with higher photon energies, except at energies, where a new shell is available. There, the cross section has a step. For $\mathrm{MeV}$ photons, the cross section depends on the $4^{\text {th }}$ or $5^{\text {th }}$ power of the atomic number $Z$ of the material.

\section{Compton scattering}

Compton scattering is the scattering of a photon with a free electron. In material this is given, if the photon energy exceeds the binding energy. The photon transfers energy to the electron, depending on the scattering angle. If the photon is back scattered, the electron will receive the maximum amount of energy, resulting in the Compton edge in the energy spectrum of the electron.

\section{Pair production and bremsstrahlung of electrons}

In this process, the photon converts into an electron-positron pair. The threshold for this interaction is a bit higher than the sum of the masses of electron and positron, because for energy conservation, a third body has to take the recoil. In matter, this is typically a nucleus.

This process is related to bremsstrahlung of an electron ${ }^{2}$ in the vicinity of a nucleus, meaning radiation of photons from the electron. The Coulomb field of the nucleus is needed for this reaction. The radiation length $X_{0}$ is the distance where the electron has only $1 / e$ of its original energy. The cross section for pair production and bremsstrahlung scale with $Z^{2}$ and the mean free path of pair production, $\lambda_{\text {pair }}$, and the radiation length are connected:

$$
\lambda_{\text {pair }} \approx \frac{9}{7} X_{0} .
$$

A high energetic photon can result in an electron-photon shower in material generating electrons and positrons via pair production, which again produce photons via bremsstrahlung. This will reduce the energy of the particles every interaction until the threshold for pair production is reached and other interactions get dominant.

In Figure 4.2, the different interactions of photons with materials are shown for the example of lead. As explained above, the photoelectric effect is dominant for low energies in the $\mathrm{keV}$ range, the Compton scattering for medium energies around one $\mathrm{MeV}$ and the pair production above one $\mathrm{MeV}$.

\subsection{Silicon Sensors}

At the moment, silicon is the standard material for tracking detectors at high energy experiments. It is also chosen for the ITk upgrade of ATLAS.

\footnotetext{
${ }^{2}$ or positron but for readability only electrons are mentioned
} 


\section{Solid State Pixel Detectors}

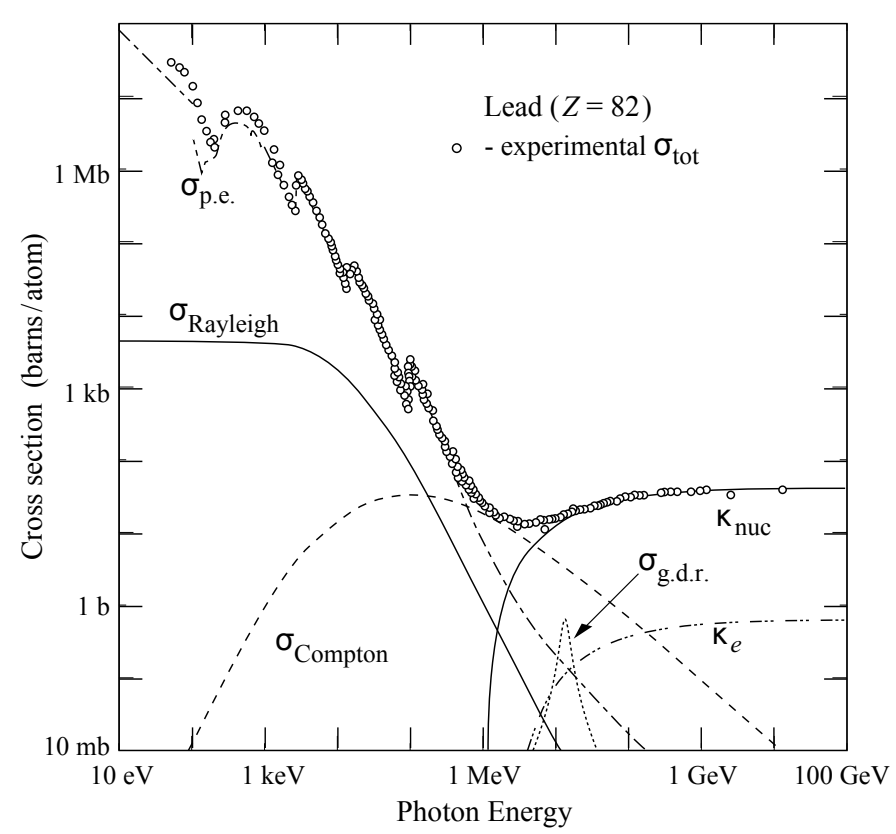

Figure 4.2.: Cross section of different photon interactions in lead [31].

Silicon is a semi-conductor, which is described via the band model with a valence band and a conduction band, separated by a gap. A passing through particle can ionize or excite electrons into the conduction band, leaving holes in the valence band. Both charge carriers can move independently in the material, but they can recombine easily.

After creation, charges move by diffusion or drift. Diffusion spreads the electrons and holes uniformly from their point of creation. Collisions reduce their energy until they recombine. For drift, an electrical field $\vec{E}$ is needed. The electrons and holes are accelerated along the field lines. Collisions with the atoms reduce the velocity and lead to a saturation velocity. The mean value of the velocity distribution is called drift velocity $\vec{v}_{\mathrm{D}}$. Even if drift is dominant, diffusion is always happening at the same time. So a drifting charge cloud spreads also in other directions. This has more influence the longer the drift lasts.

A signal is not realised when the charges reach the collecting electrodes, but during the drift. The moving charges $q$ change the electrical field and induce a current on the electrodes. This current is described by the Shockley-Ramo theorem $[35,36]$ :

$$
I_{\text {ind }}=q \cdot \vec{E}_{\mathrm{w}}(r(t)) \cdot \vec{v}_{\mathrm{D}}(r(t)) .
$$

The weighting field $\vec{E}_{\mathrm{w}}$ represents the effects of the geometry of the device for the electrical field.

To reduce the intrinsic amount of electron hole pairs in the silicon and thus reducing the leakage current, the silicon is doped. In the doped silicon, other atoms replace silicon atoms in the lattice, introducing acceptors for electrons (p-type) or donors of additional 
electrons (n-type). Acceptor atoms have one electron less than silicon and donor atoms one more. If silicon is p-doped on the one side and n-doped on the other, forming a diode, the extra electrons neutralize the holes, where the zones of different doping meet. This results in a so-called depletion zone, in which an electric field is formed by the atoms which are no longer neutral because of the charge exchange. This zone is in equilibrium with the rest of the material. An external bias voltage can be applied to widen the zone by shifting the equilibrium.

Electrons and holes created in the depletion zone drift away from each other due to the electric field in the depletion zone, and can hence induce a signal on the electrodes on the surface of the material. Normally, one aims for a completely depleted detector to get the most of the signal and a fast signal, but there are other methods which only need partial depletion [37].

To measure the track of a particle, multiple layers of silicon detectors are needed, every one providing one point on the track. For this, it is important that the passage through one detector does not significantly alter the way of the particle. This can happen through the energy loss or multiple scattering. So the amount of interacting material has to be low, without losing too much of the signal, even though electron-hole pairs can be created.

\subsubsection{Radiation damage}

Lattice atoms can be displaced from their position by interacting particles. This creates defects in the material, such as vacancies and interstitials. A knocked off atom can produce more defects, resulting in cluster defects, if it has enough energy. The so induced modifications to the material can change the signal of the particles to detect. Here, effects on silicon are discussed, but the behaviour is similar in diamond [38-40].

The defects in silicon create new energy levels between the valence and the conduction band. Levels in the middle of the band gap give rise to increased leakage current, as thermal excitations get more likely. This effect can be reduced by applying cooling to the detectors.

Trapping of electrons or holes can happen at levels near the bands. After some time the trapped charges are released, but by then, the signal has been usually already integrated without these charges. So the signal is decreased or even interferes with a following signal.

In silicon, the defects are mostly acceptor-like, resulting in a change of effective doping concentration. At high particle flux, an n-type sensor can even effectively be inverted to a p-type. With higher effective doping concentration in the sensor, a higher bias voltage has to be applied to completely deplete it. As the sensors are essentially diodes operated in reverse bias mode, the breakdown voltage of the sensor limits the ability to deplete the detector. 


\section{Solid State Pixel Detectors}

\subsection{Diamond Sensors}

Diamonds have interesting physical properties. These make them an option for tracking detectors. In the ATLAS detector, the beam monitor consists already of diamond sensors and another tracking beam monitor has been installed with the IBL upgrade [16].

In this section, first, the properties of diamond are displayed and compared to silicon, then, the production of diamonds is explained. At last, a method is introduced to produce 3D diamond pixel detectors. The content is focused on the basic concepts. A comprehensive summary about this topic with far more detailed explanations can be found in [41].

\subsubsection{Diamond Properties}

Diamond is made of carbon atoms. The four valence electrons of the atom form $s p^{3}$ hybrid orbitals. Overlapping orbitals of neighbouring atoms create stable bounds and form the diamond crystal. In Table 4.2, some properties are listed for diamond and silicon, which will be discussed in the following.

The comparison of diamond with silicon is a valid choice, because the mechanism of signal creation by ionisation is the same for silicon and diamond. Their electron structure can be described with the band model of valence and conduction bands. Also, silicon is the standard material for tracking detectors at present. The production of silicon is industrially very well established with a high purity. Silicon itself has many options of how to build a detector, whether it is a monolithic sensor with integrated readout electronic or a hybrid with separate sensor and read-out. So in every application diamond has to compete with silicon.

\begin{tabular}{lcc}
\hline Property & Diamond & Silicon \\
\hline band gap $[\mathrm{eV}]$ & 5.5 & 1.12 \\
energy for a $e / h$-pair $[\mathrm{eV}]$ & 13 & 3.6 \\
radiation length $[\mathrm{cm}]$ & 12.2 & 9.4 \\
mean signal $/ \mu \mathrm{m}[\mathrm{e}]$ & 36 & 89 \\
intrinsic charge carrier density $\left[\mathrm{cm}^{-3}\right]$ & $<10^{3}$ & $1.5 \times 10^{10}$ \\
breakdown field $[\mathrm{V} / \mathrm{cm}]$ & $10^{7}$ & $3 \times 10^{5}$ \\
resistivity $[\Omega \mathrm{cm}]$ & $>10^{11}$ & $2.3 \times 10^{5}$ \\
dielectric constant & 5.7 & 11.9 \\
density [g cm $\left.{ }^{-3}\right]$ & 3.52 & 2.33 \\
dislocation energy $[\mathrm{eV} /$ Atom] & 43 & $13-20$ \\
\hline
\end{tabular}

Table 4.2.: Properties of diamond and silicon [42].

Diamond is an insulator, in contrast to silicon, which is a semi-conductor. This classification results from the high band gap for diamond of $5.5 \mathrm{eV}$ and the low gap of $1.12 \mathrm{eV}$ for silicon. This implies that a higher energy is needed to create an electron-hole pair 
in diamond, which is 3.6 times higher than in silicon. Also, the resulting signal per micrometer material is less than half than in silicon.

Silicon detectors are used as a diode with a p-n-junction as described in Section 4.2. They need a bias voltage to deplete the sensor of intrinsic charge carriers. The density of those in diamond is seven orders of magnitude lower, thus a depletion voltage is not necessary. But to collect the signal, a voltage has to be applied to get reasonably short collection times by increasing the drift velocity and not relying on the diffusion process.

The mobility of the charge carriers is intrinsically high in diamond, resulting in fast signals as needed for high particle flux environments [43]. The higher breakdown field and resistivity of diamond allows to use high bias voltages. With high electric fields, the drift velocity of the electrons and holes rises, leading to even faster signals.

But not only the creation of signal is important for a detector, also the noise, or better the signal to noise ratio, is the crucial property. The electrical noise induced from the sensor capacitance is lower for diamond, because the dielectric constant is only half of that of silicon.

In addition to this, the high band gap for diamond is useful, because thermal excitations with very high energy are rare. This leads to a small leakage current, in comparison to silicon. The leakage current is Poisson distributed and its noise therefore proportional to the square root of the current [39]. So, a smaller leakage current means less noise. To reduce leakage current, cooling can be used so that thermal excitation is suppressed. If there is less leakage current intrinsically in the sensor, less cooling is needed.

The thermal conductivity of diamond is high [44], so cooling is easier and not the whole sensor has to be directly cooled. It could be sufficient to put the cooling next to the sensor, because it can be better distributed. This reduces the cost and the space requirements of the detector.

For high luminosity experiments, the particle flux is high near the interaction point. This is where the tracking detectors are placed for good position measurements of the different vertices. Consequently, radiation hard materials are required. Diamond has a higher dislocation energy for its atoms than silicon. Hence, the damage done to the material from a particle in diamond should be less than in silicon. Especially consecutive damage from primary knock on atoms should be reduced.

One major issue is the trapping of charges in diamond, i.e. electrons and holes get trapped in a long living state and do not contribute to the signal. Those states are introduced by radiation damage or intrinsic flaws in the lattice. Polycrystalline diamond has many grains and at their boundaries the lattice is disturbed because they do not line up properly. The property describing the mean distance which an electron and a hole separate by drift in an electrical field is the charge collection distance, CCD. This distance is greatly influenced by the amount of traps as the mobility $\mu$ and the lifetime $\tau$ of the charge carriers are used to calculate it in a given electric field $E$ :

$$
\mathrm{CCD}=\left(\mu_{e} \tau_{e}+\mu_{h} \tau_{h}\right) E .
$$

Altogether, diamond is an option for tracking detectors at high flux particle experiments. It has a smaller signal than silicon but with low noise this is not a problem. High 


\section{Solid State Pixel Detectors}

particle rates can be observed because the charge collection in diamond is very fast.

\subsubsection{Synthetic Diamonds}

The properties of natural diamonds are not reliable and they are not cheap either. Also, they vary strongly in size and purity. Hence, they are of no use for high precision applications. For this reason, the industrial production of diamonds is growing and developing fast. One method to synthesise diamond is chemical vapour deposition (CVD) [44]. For this, pressures of few to tens of kilo pascals and temperatures of 700 to $1200{ }^{\circ} \mathrm{C}$ are needed, which is orders of magnitude lower than natural genesis or other methods as the high pressure, high temperature (HPHT) one [45]. In this region, graphite is the stable conformation of carbon and not diamond. Graphite forms $s p^{2}$ orbitals and is electrical conducting. To get diamond, the composition of the used gas in the process is of importance.

The gas is a mixture of hydrocarbon, e.g. methane, and hydrogen. The hydrogen leads to a sealed surface of hydrogen with five-member rings of carbon beneath. Hydrogen from the gas phase collides with the surface, stripping some of the hydrogen away. This creates reaction possibilities for the hydrocarbon and its radicals to form in the next step a six carbon atoms ring, the diamond bulk. Graphite is also produced, but the hydrogen etches it away in a higher rate than diamond.

The growth of diamond starts on a given substrate which determines already some properties of the diamond. At random positions the growth starts. These diamond seeds have the same orientation as the substrate at this place. Some orientations lead to a faster growth than others, leading to bigger grains with this orientations. Singlecrystal diamond (scCVD) can only be obtained by using another single-crystal diamond as substrate. So all grains start with the same orientation and can form a single-crystal. For poly-crystals (pCVD) a silicon sample is sufficient.

The difference between pCVD and scCVD can be seen in Figure 4.3. ScCVD diamonds have only impurities from the production, e.g. resulting from contamination of the gas. But pCVD diamonds grow in different grains, starting very fractioned at the seed surface and ending in bigger grains on the growth surface. Observations have been made that the boundaries of the big grains are more or less perpendicular to the growth surface. But even the bigger grains contain smaller structures [46].

The scCVD diamonds have a higher CCD than the pCVD diamonds because of less impurities in the single-crystal. Typical values are $500 \mu \mathrm{m}$ for scCVD diamonds and $250 \mu \mathrm{m}$ for pCVD diamonds at applied fields of $1 \mathrm{~V} / \mu \mathrm{m}$. But with irradiation, the CCD decreases exponentially with fluence for both with the same damage constant [47]. So scCVD diamond does not have a better response after high particle fluencies, only a better starting point.

The production of scCVD is limited by the substrate size and the growth rate. With higher growth rates more impurities are created. This limits the efficient production of scCVD diamonds. The pCVD diamonds can have areas in the order of $\mathrm{cm}^{2}$ and are cheaper. But they also have large amounts of impurities. So the tests of pCVD diamond 


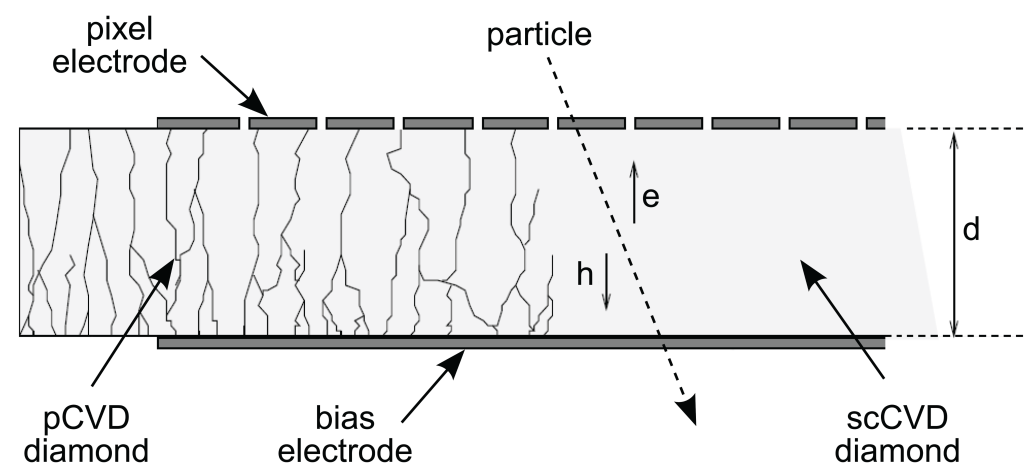

Figure 4.3.: Schematic of sc- and pCVD diamonds.

for detector applications are important and can lead to larger instrumented areas with a reasonable expense, if pCVD properties are good enough.

\subsubsection{D Diamond Sensors}

As mentioned above, trapping of charge carriers while drifting to the collecting electrodes is a problem with diamond sensors. In pCVD diamond this is even more relevant because of the grain boundaries and impurities. Over the lifetime of the detector induced damage by radiation degrades the signal collection even further. During operation, the CCD can be recovered by increasing the bias voltage, but there are technical limitations beyond which this is not applicable. Reducing the drift distance of the charges is the remaining option. This distance is defined by the placement of the electrodes. For silicon and diamond detectors they are commonly placed at opposing surfaces of the sensor. Evaporated metal layers on the surfaces are used to segment the planar diamond detectors. Accordingly, thinner sensors improve the charge collection. But with less material a lower overall signal is created, and the sensor is mechanically more unstable.

An alternative to electrodes on the surface are electrodes in the bulk material itself, perpendicular to the surface, see illustration in Figure 4.4 [48]. This decouples the spacing of the electrodes and the sensor thickness. The latter one can then be chosen to match the signal strength. In this so called 3D geometry, the electrodes can be as close as some tens of micrometres. Trapping probability is reduced as the CCD of the material is at least in this order of magnitude. Other advantages are a lower bias voltage needed for the same electrical field, because of the short distances between the electrodes, which leads to a faster signal collection. The $3 \mathrm{D}$ technique would make the use of $\mathrm{pCVD}$ diamond viable.

\section{Graphitisation of Diamond}

Implementing a 3D geometry in silicon sensors can be done via etching of holes and filling with electrode material. Diamond is very stable and chemically inert. A different approach is taken by using a femtosecond laser to induce a phase transformation of the 

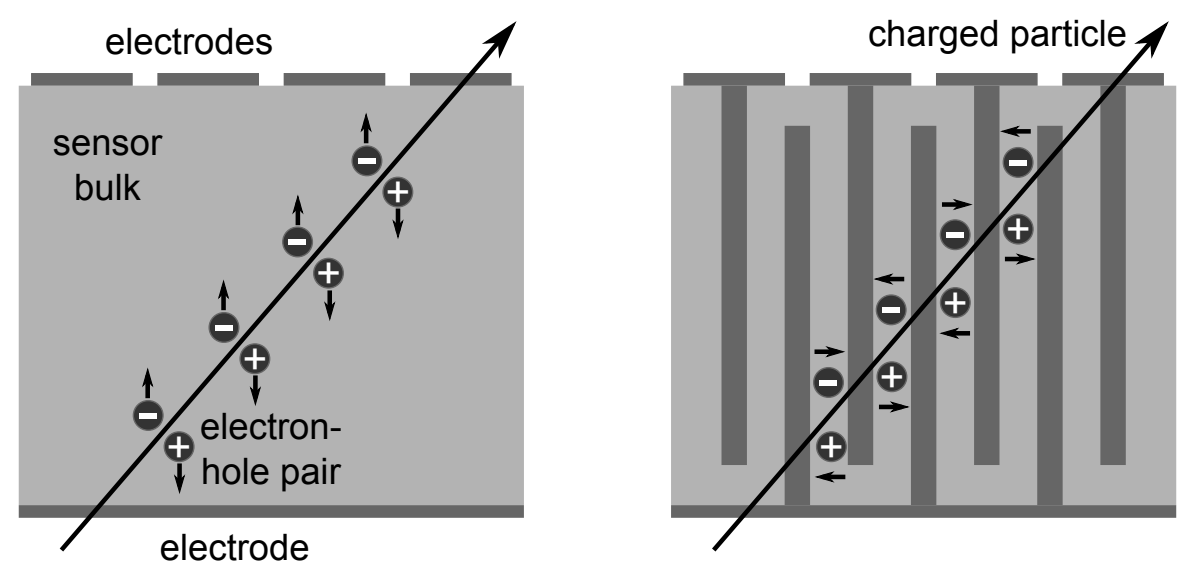

Figure 4.4.: Schematic of a planar (left) and 3D (right) electrode geometry. Thickness of $3 \mathrm{D}$ electrodes and sensor are not to scale.

carbon atom bonds in the diamond crystal [49]. Changing from $s p^{3}$ to $s p^{2}$ hybridisation yields electrically conducting material. Fully ordered $s p^{2}$ material is called graphite.

Diamond is transparent for the typical femtosecond laser wavelength [50]. But each pulse has high power and high fluence of photons, making multiphoton ionisations possible [51]. Electrons get excited into the conduction band and the atoms finally ionised. The crystal heats up because of collision of these electrons with other atoms in the vicinity. If a certain energy is reached in the crystal, the $s p^{3}$ bonds change to $s p^{2}$ [52].

The threshold energy depends on the surrounding material. In the bulk it is the highest, lower at surfaces, and even lower if phase changed material is already present [50]. Shining a focused laser into a diamond results in spontaneous phase change at locations where the threshold is exceeded. Graphite structures absorb the energy and block further changes downstream of the laser. Continuous columns can be produced if the energy upstream of a graphite spot is too low for transformation in the bulk, but high enough for transformation near the existing changed material [49].

Controlled production of continuous electrodes in diamond is easiest achieved by moving the sample through the laser focus [53]. The lower threshold at the surface helps to create a seed for the electrode. Upstream, the threshold for bulk transformation should not be exceeded to guaranty continuous growth. Because of the high repetition rate of the laser pulses, at the exit surface the laser will shine on already transformed material and burn it away. The formation of crater like structures can happen.

With the help of improved optics, the process can be optimised. Using so call Spatial Light Modulation (SLM) modules, the phases of the laser can be controlled and the focus reduced $[54,55]$. The corrections can be made dynamically, depending on the position of the focus in the diamond. This reduces the width of the electrodes, but also allows reprocessing them multiple times, because of a wide incident angle of the focus. The internal structure of the electrode can be improved by removing remaining diamond material. 
To contact the columns for readout and supplying bias voltage, they are metallised at the surface. In a photo lithographic process, first, $50 \mathrm{~nm}$ chromium and then, on top, $100 \mathrm{~nm}$ gold are deposited. Deep craters are problematic as the walls mostly consist of diamond material and the graphite at the bottom is not necessarily reached.

\subsection{Hybrid Pixel Detectors}

The focus of the last sections was on the material properties of sensors. Here it is introduced how they are used to form a position resolution detector on the example of a hybrid pixel detector.

The first point is position resolution. For this, a segmentation of the sensor is needed to be able to distinguish, where a particle passed through the material. Dividing it in two dimensions across the surface forms pixel. The resolution of a simple binary readout can be calculated to be pitch $/ \sqrt{12}$, where the pitch describes the pixel spacing. If information about collected charge is recorded, weighting algorithms can be applied to improve the position estimation. Using multiple detectors behind each other allows to measure the trajectory of a particle in space.

In silicon, a pixel can be produced by dedicated doping of the bulk material and the individual pixel area. Between the bulk and the pixel implants, p-n junctions are formed. This is a very simplified picture, which gets only more complicated if radiation hardness of the structures has to be considered. For diamond, the pixelation is achieved with the pattern of the surface metallisation.

In case of 3D electrode geometry, an intrinsic segmentation is provided for both silicon and diamond. Connecting the electrodes to either the readout or the field, generating bias voltage forms the pixel. Different shapes and different number of collecting electrodes are possible. Electrodes extending not completely to the other side of the bulk can simplify the connection by physically separating the different types of electrodes to the two surfaces. For diamond, this entails a very exact start and stop of the laser focus position inside the material. Double sided processing is possible for silicon, but increases the complexity of the fabrication.

Until now, only the creation of signal by particles in the material has been considered, but also reading it out and storing it is important. Implementing electronics and logic inside silicon is a standard process. This is used in monolithic detectors, where the readout is already a part of the sensor design. Separating sensor and read-out is described with the concept of hybrid detectors. Both parts can be individually optimised and adapted to the needs. For pixelated detectors, the individual sensor pixels are then connected to corresponding read-out pixels. This is done by so called bump bonds, small tin silver or indium connections. As this is an extra process step compared to monolithic designs, the complexity and cost increases in general.

In this thesis, the detectors under study are hybrid detectors. Because the RD53A chip, prototype of the read-out chip for the ITk, was not available, the ATLAS FE-I $4 \mathrm{~b}^{3}$

\footnotetext{
${ }^{3}$ As only the $\mathrm{b}$ version is used, the $\mathrm{b}$ is dropped in this thesis
} 


\section{Solid State Pixel Detectors}

chip is used, which was designed for the ATLAS IBL [56]. It has a pixel matrix of $80 \times 336$ organised in columns and rows with pitches of $250 \mu \mathrm{m}$ and $50 \mu \mathrm{m}$, respectively. In Figure 4.5, the pixel electronics is displayed. The signal from the sensor pixel gets first shaped from a current to a voltage signal. Then it is amplified and discriminated to suppress noise. With the discrimination the time over the threshold (ToT) of the signal is measured. The circuit is designed such that this value is proportional to the deposited charge in the sensor.

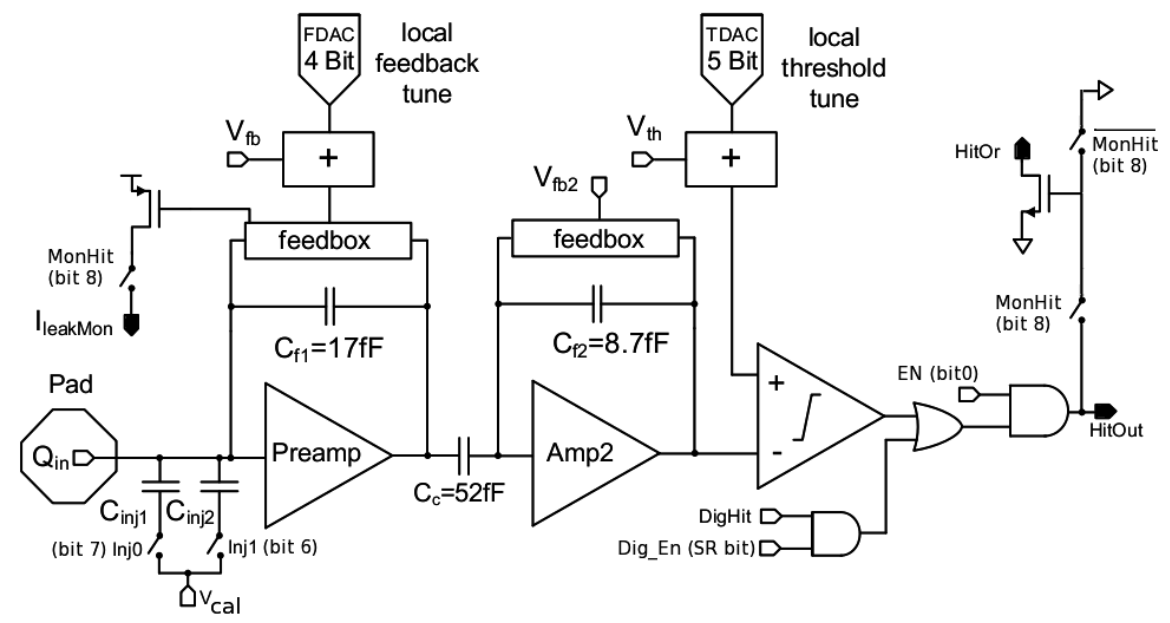

Figure 4.5.: Schematic of the FE-I4 readout pixel [57].

The individual components of the read-out pixel can be calibrated for the whole chip and per pixel. A circuit for injecting test charges in the different stages is available. From the response, the global and local pixel registers can be tuned.

The FE-I4 has to be powered with a digital and an analogous voltage of $1.2 \mathrm{~V}$ and $1.4 \mathrm{~V}$, respectively. These voltages can also be generated by an Shunt-LDO circuit implemented in the chip, when it is supplied with a constant current. The voltages can be regulated by registers in the chip. This allows for serial powering chains with the FE-I4 chip. 
CHAPTER 5

Module Assembly and Testing

On the way towards the ITk, many studies on all the parts are conducted. Larger scale tests are done in the so-called demonstrator projects. The work presented here, is carried out in the framework of the FE-I4 demonstrator stave at CERN. It targets the outer barrel layers of the pixel detector in ITk. Tools for building the pixel modules are developed and test results presented here. The main focus is the alignment of the different parts and the wire bonding.

Modules produced for ITk have to be of high quality. This is controlled by testing every module mechanically and electrically. A selection of tests is set up for and with the prototype modules of the demonstrator, and the results are presented. For the actual production phase, a test system is needed to ensure the standard and the timely execution of the tests. A prototype of such a system is developed and described and qualified here.

The tools and processes developed for the building of the modules are presented in Section 5.1. Tests carried out with the produced modules and the test stand are the content of Section 5.2.

\subsection{Module Assembly}

In this section, first the components of a module, then the processes and tools used to build the modules are described. The quality of the tools is assessed, and problems that occurred during the assembly and possible solutions are discussed. While the specifics of the modules are for the demonstrator, the processes and tools are developed for the ITk production. 


\section{Module Assembly and Testing}

\subsubsection{Hybrid Pixel Module}

A schematic of the module stack is depicted in Figure 5.1. The read-out chips, 2 or 4 for double or quad chip modules, respectively, are bump bonded to a sensor with matching size. This is then called a bare module. A flexible PCB, flex hybrid or flex, is glued on top. It provides the connections to the read-out system and power supplies. Aluminium wire bonds rout voltages and signals between the flex and the read-out chip and sensor. To protect the wire bonds mechanically and from corrosion, they are encapsulated. The resulting modules are called double or quad chip modules according to the number of read-out chips connected to the sensor. This module concept follows the principles of the IBL modules [16]. The specific components, that are used for the Demonstrator modules, are hereafter described.

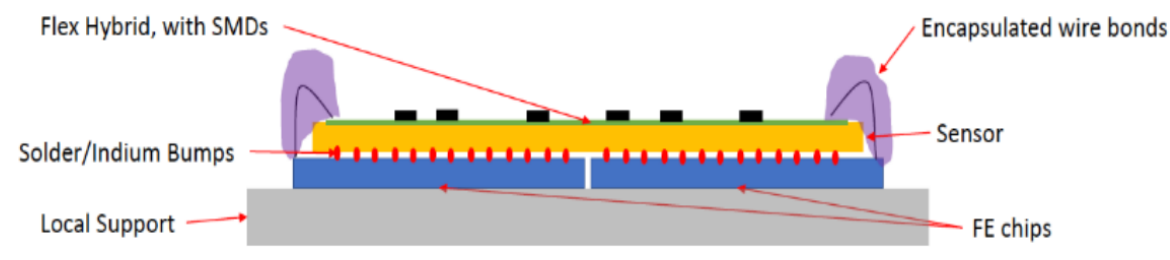

Figure 5.1.: Schematic of a module [21].

The sensors are made of n-in-p and n-in-n high resistivity silicon for the quad and the double chip modules, respectively. As a read-out chip the ATLAS FE-I4, currently in operation in the IBL, is used. For a description see Section 4.4. The sensors are $200 \mu \mathrm{m}$ thick and the read-out chips $450 \mu \mathrm{m}$ thick. The sensor and read-out chips are connected with Silver-Tin bump bonds pixel by pixel in an industrial process.

The flex is a multi layered circuit on a flexible PCB material. Wire bond pads and rails are implemented at the side of the flex main part towards the chips. The rails distribute ground, digital and analogous potential for the read-out chips. Two holes in the inner part of the flex allow for the connection of the bias voltage to the sensor with wire bonds. To monitor the temperature of the module, two NTC resistors are mounted on the flex. For every read-out chip, test pads are provided on the flex to access the digital and analogous voltage generated by the shuntLDOs from the supplied current. The connector to the support structure is located on a tail that is designed to be bent to fit in the demonstrator stave structure. In Figure 5.2, a schematic of the quad chip module flex and a picture of such a flex is shown, and in Figure 5.3 the same for a double chip module. The flex comes with a frame for easier handling and stability. It is connected with small bridges to the main part and the tail. For alignment of the quad (double) flex, the five (six) holes in the frame with a diameter of $3.4 \mathrm{~mm}$ are used.

\subsubsection{Assembly Tooling}

The concept of the tooling is based on the IBL production design and is further developed for the ITk production [58]. It accomplishes the alignment of the flex to the bare module 


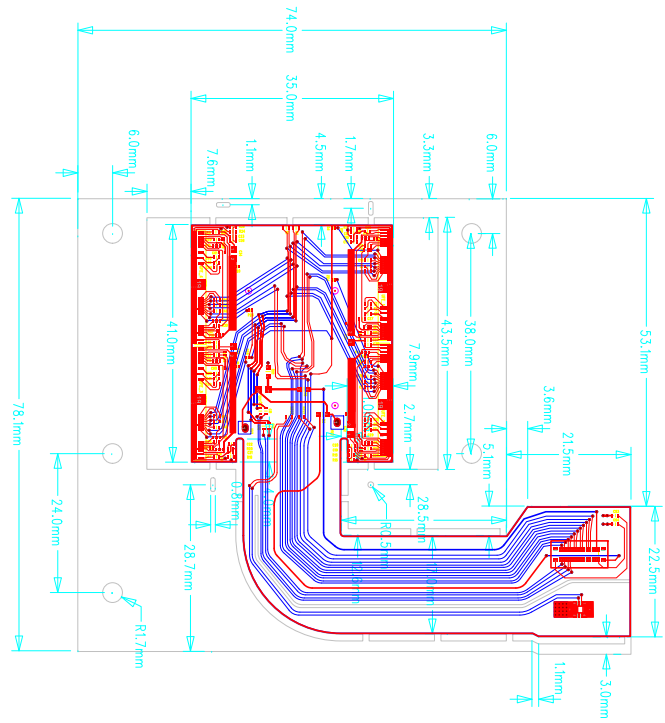

(a)

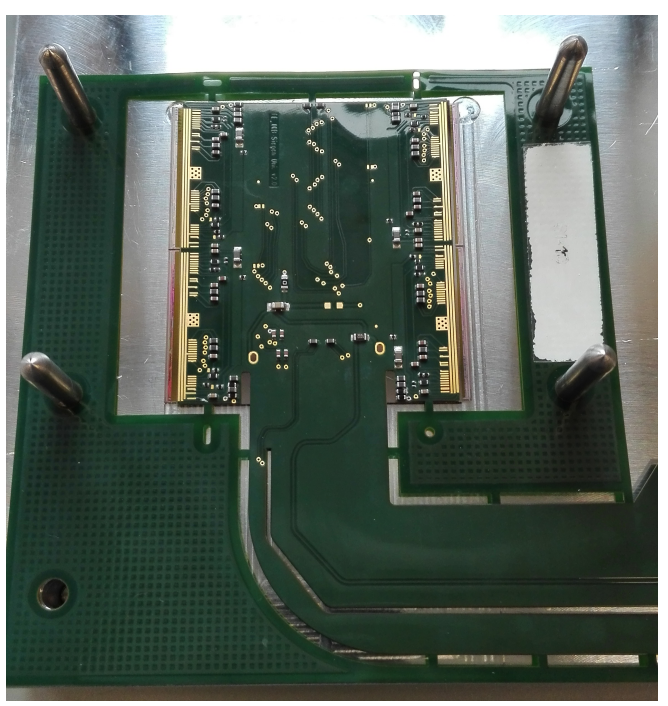

(b)

Figure 5.2.: Schematic and picture of a quad chip module flex for the demonstrator.

so they can be glued together. The assembly tool is designed with the goal to carry out all steps on this same tool, because moving the module is the most critical step, because it is so fragile. It can be fixed on the tool which is matched to all workstations.

The main part is the aluminium jig with an extrusion of $200 \mu \mathrm{m}$ depth on which edges the bare module is aligned by pushing it into one corner, see Figure 5.4(a). Holes in the extrusion area allow to fixate the bare module with under pressure generated by a pump connected to the jig. For the positioning of the flex on the bare module, holes and dowel pins $^{1}$ in the jig are used matching to the holes in the flex frame. While two hole and pin positions are enough to position the flex, up to six holes are manufactured in the jig to be able to constrain the flexibility of the flex and prevent bending. No over constraints are observed in the test. The pins are made of stainless steel for higher durability, and are removable to not obstruct the space for other machines like the wire bonder and microscope oculars for visual inspections.

A plexiglass spacer with cut outs for the components of the flex is used to apply an even pressure to the module during the curing of the glue, see Figure 5.4(b). It is loosely positioned with the pins. Additional weights can be put on top as needed. Later in the process it can be used as protection especially for the wire bonds. That is why a large area around the wire bond pads and the read-out chip pads is also left open in the spacer.

The tolerances on the lateral alignment of the flex and bare module are mainly driven by the semi-automatic wire bonding machine, so that not large displacements have to be manually corrected in the programs for the wire bonding. For the quad chip modules

\footnotetext{
${ }^{1}$ Shortened to pins in the following text.
} 


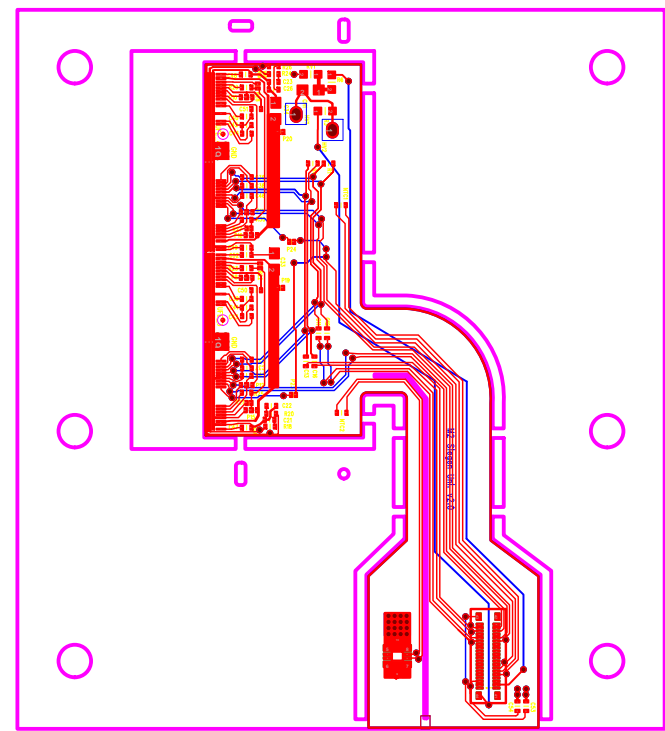

(a)

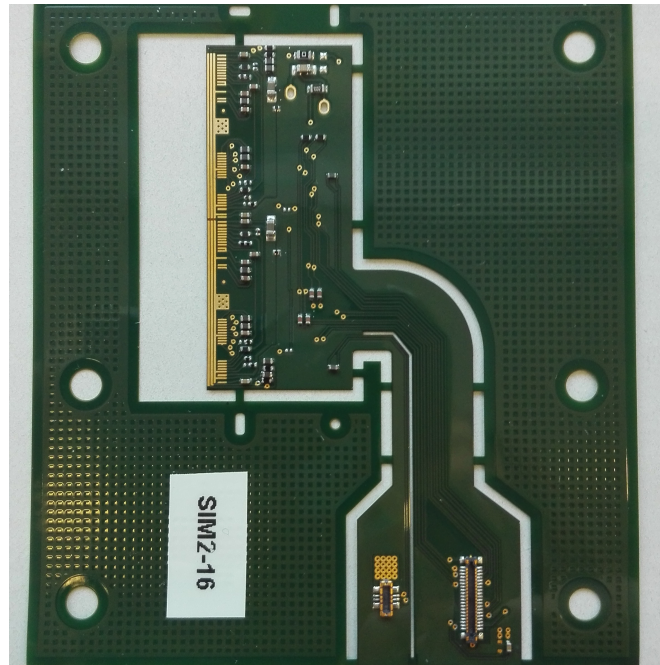

(b)

Figure 5.3.: Schematic and picture of a double chip module flex for the demonstrator.

tolerances of $100 \mu \mathrm{m}$ are required for the Demonstrator. Because of constraints of the space to load the double chip modules on the stave, only one option remained to position the flex and the tolerance had to be lowered to $50 \mu \mathrm{m}$ for the double chip modules. In the ITk production the tolerances will be also $50 \mu \mathrm{m}$ for the alignment.

\subsubsection{Assembly Procedure}

The assembly starts with the bare modules. The read-out chip bump bonding to the sensor is done by an industrial partner, and the flexes are also industrially produced. At the moment, the electrical components are loaded onto the flex by the institutes, but in future this will be done in industry. The assembly process can be divided into three steps: preparation of components, alignment and glueing, and wire bonding.

\section{Preparation of Components}

All components, bare module and flex, have to be visually inspected for defects, scratches, dimensions, solder residue, and electrical components, to name only a few. Faulty components are excluded from the assembly and possible damage from transportation between sites can be discovered. Then the flexes are cleaned in an ultrasonic bath with a $10 \%$ Vigon N600 solution to remove residues like halogens from the solder process that might corrode the wire bonds later, and to ensure a clean surface for the wire bonds. After the first bath the flexes are rinsed with de-ionised water in a second ultrasonic bath for a short time to remove the Vigon. The remaining fluid is first dried with dry air or nitrogen gas and then in an oven. 


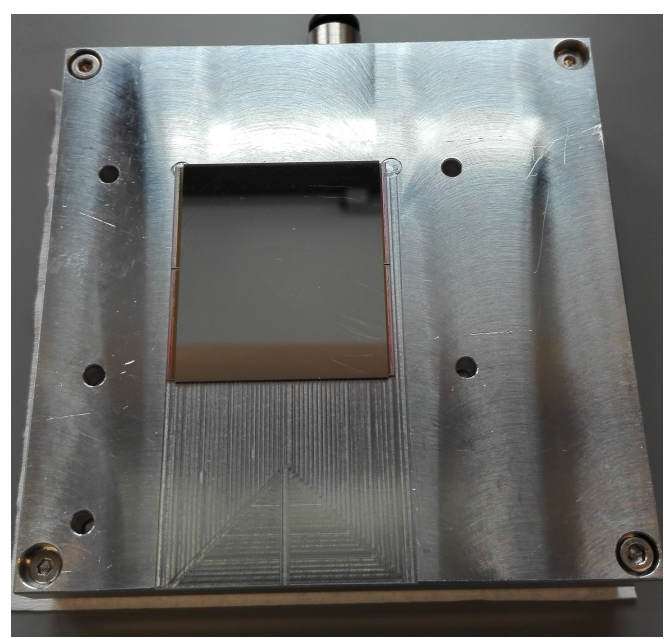

(a)

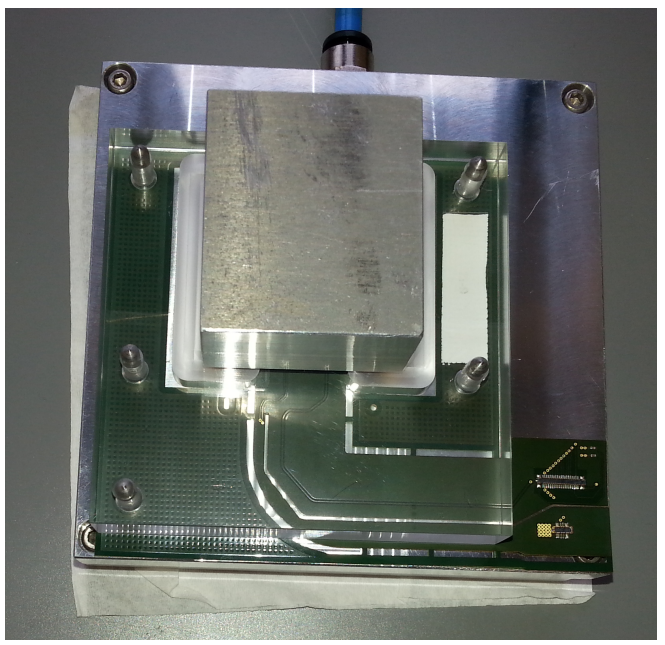

(b)

Figure 5.4.: (a): picture of a quad chip module jig with a bare module positioned on the edges. (b): Picture of a quad chip module during the glue curing with weight applied.

\section{Alignment and Gluing}

In this step, the flex is glued to the bare module. Two glues are used, the one is a standard epoxy glue, and the other on is a double sided silicone adhesive tape ${ }^{2}$. Figure 5.5 shows the glue pattern. The tape is used to ensure a stable and flat surface below the wire bond pads. When distributing the epoxy glue, care has to be taken that the glue does not ooze through the vias in the flex. If this happens, other parts of the tooling have a high chance to adhere to the module. Removing these parts needs force and might damage the module.

The most crucial part of the gluing step is the alignment of the flex to the bare module. The bare module is positioned at the edges of the extrusion in the jig, and with the pins the flex with the glue applied is lowered onto the bare module. Up to six stainless steel pins are used, which constrains the position of the flex well. Vacuum suction holds the bare module stable on the jig during the whole process.

With the flex in position, the glue has to cure. As the adhesive tape has a short cure time of a couple of minutes, a good contact has to be ensured by pressing down the flex. After that, the plexiglass spacer and a weight of total $150 \mathrm{~g}$ is put on top for the cure time of the epoxy of nine hours, see Figure 5.4(b).

Inspecting and measuring the alignment of the components are done after the glue is cured. Then the module can be wired bonded. For this the module can be kept on the aluminium jig, as it is designed to fit the workspace of the wire bonder.

\footnotetext{
${ }^{2}$ Epoxy: UHU Plus Endfest for availability. Tape: PPI-RD 577F from IBL production
} 


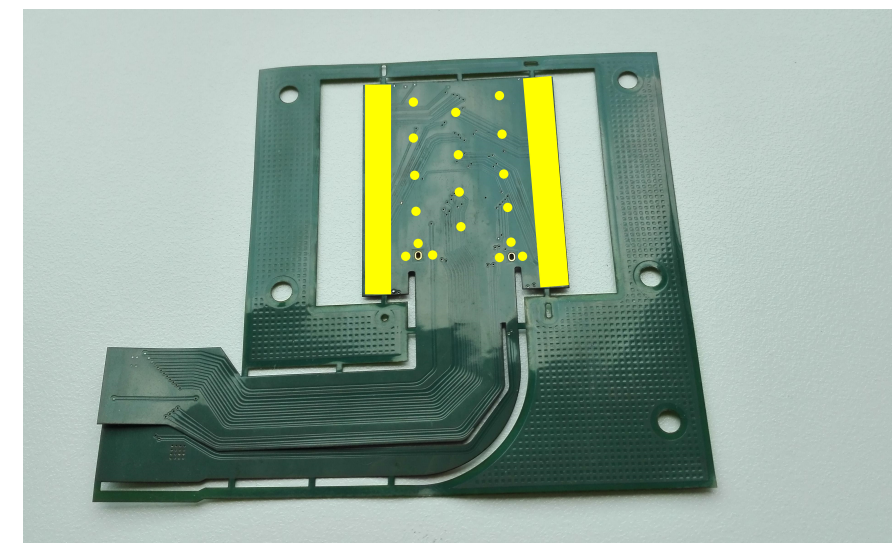

Figure 5.5.: Picture of a quad chip module flex backside with the glue pattern indicated in yellow, dots for epoxy glue and strips for adhesive tape.

\section{Wire bonding}

The flex and the read-out chip get connected with aluminium wires of $25 \mu \mathrm{m}$ diameter. Every FE-I4 chip needs about 100 wires, the pattern is depicted in Figure 5.6. Special care is taken in the order of the wire bond process to prohibit electrostatic discharges by connecting the reference voltages and ground first before other wire bonds. To compensate for production differences, a set of four wires are used to set the current reference to $2 \mu \mathrm{A}$ per read-out chip. The chip id has to be set with a binary combination of three wires to distinguish the read-out chips. In addition, high voltage is connected through holes in the flex to the sensor.

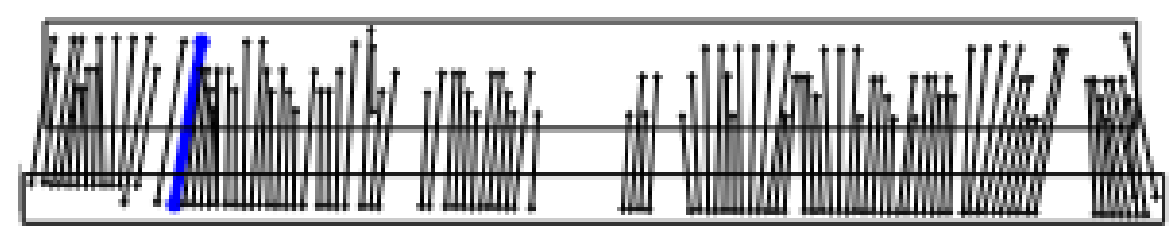

Figure 5.6.: Wire bond pattern for a FE-I4 chip to the quad module flex.

A semi-automatic wire bonder from BondTech with the 5630 wedge tool module is available in the laboratory. It is possible to program and save the position of many wires in relation to layout defining adjustment points. Pattern recognition is available to find the adjustment points and speed up the set up of a new module on the workbench. The wires can be automatically bonded in the programmed order.

A wire is attached to the wire bond pad on the flex and then to the pad of the read-out chip by using a force and ultrasonic movement of the wedge bond tool. In Table 5.1, the most relevant parameters on the adhesion of the bond foot to the surfaces and the optimised values for this module production are listed. They control the force used with 
the bond tool, the power of the ultrasonic movement and the duration of the bonding process. The values are quite different between the flex and the read-out chip, and the adhesion on the flex is in general more difficult as can be seen from the need for more powerful settings for the flex. This is expected given that the connection of the aluminium wire is easier to the aluminium pad on the read-out chip than to the gold finished pad on the flex. Also, the surface of the flex pads is visually rougher than the read-out chip pad, and the flex surface might not be as stable under force due to the flexibility of the material.

\begin{tabular}{|l|c|c|}
\hline Parameter & Flex & FE-I4 \\
\hline \hline Bond time [ms] & 50 & 40 \\
\hline US power [digits] & 220 & 180 \\
\hline Bond force [g] & 50 & 35 \\
\hline
\end{tabular}

Table 5.1.: Most relevant parameters for the adhesion of wire bond foots with the specific parameters for the flex and the FE-I4 surface.

\subsubsection{Quality Assessment}

The tooling was produced by the central workshop of the faculty of physics in Göttingen. Here, tests are presented that assess the quality of the tooling, and to validate the new design of the tool. Problems and solution during the assembly are discussed. The tests are carried out with one jig for quad chip modules, and six jigs for double chip modules, which were produced in two batches a one and five jigs.

\section{Tool Production}

In Table 5.2, the diameters of the holes in the different jigs and the mean diameter per batch of pins are displayed. The variation of the hole diameter between plates is with $12 \mu \mathrm{m}$ low for the batch of five double chip module jigs produced last, only one plate deviates with larger holes. But comparing to the first jig for double chip modules or even the quad chip module jig, the differences are non negligible with $40 \mu \mathrm{m}$ and $90 \mu \mathrm{m}$, respectively. The diameter of the holes is with nominal $3.4 \mathrm{~mm}$ not a standard size. For this, drills are available in the workshop but not of the high precision as it would be for $3 \mathrm{~mm}$.

For the last batch of double chip module tools, two batches of pins are produced. Using six pins of each batch, the flex SIM2-16 is placed first as far top left on the jig as possible with the pins and then shifted in the opposite direction to create the maximum distance between positions. This distance is for the first batch of pins in the range of $40-90 \mu \mathrm{m}$ depending on the jig used. For the second batch of pins this distance is significantly lower with a maximum shift measured of $(20 \pm 5) \mu \mathrm{m}$. The large differences could be partially explained, because the flex is pushed down in the later measurement 
5. Module Assembly and Testing

\begin{tabular}{|c|c|c|c|c|}
\hline Jigs & Quad & Double 0 & Double 1 & Double 2 \\
\hline Hole diameter $[\mathrm{mm}]$ & $3.568(6)$ & $3.43(1)$ & $3.50(2)$ & $3.466(7)$ \\
\hline \hline Flex & SIM2-16 & Double 3 & Double 4 & Double 5 \\
\hline Hole diameter $[\mathrm{mm}]$ & $3.425(6)$ & $3.47(1)$ & $3.466(6)$ & $3.473(7)$ \\
\hline \hline Pins & Quad & Double built & Double b1 & Double b2 \\
\hline Diameter $[\mathrm{mm}]$ & $3.39(1)$ & $3.385(3)$ & $3.378(3)$ & $3.396(4)$ \\
\hline
\end{tabular}

Table 5.2.: Mean diameter of the holes in the aluminium jigs for quad and double chip modules, the mean diameters for batches of pins, and the holes in a double chip module flex. Uncertainties are standard deviations from the distribution of measured values.

and flat, while for the first measurements the flex could be bowed as the flatness is not controlled.

The pin diameters of the two batches are displayed in Figure 5.7. The design diameter for the second batch is $3.395 \mathrm{~mm}$ to fit into the holes as well as into the flex holes, and still be able to be removed without much force, which potentially might be dangerous to the module. The consistency of the production is very good, because the standard deviation of the sets are about $4 \mu \mathrm{m}$, which is within the measurement uncertainty. The difference of the mean diameters of the batches is $(18 \pm 2) \mu \mathrm{m}$. This explains the general difference in the movability of the flex with the different batches of pins.

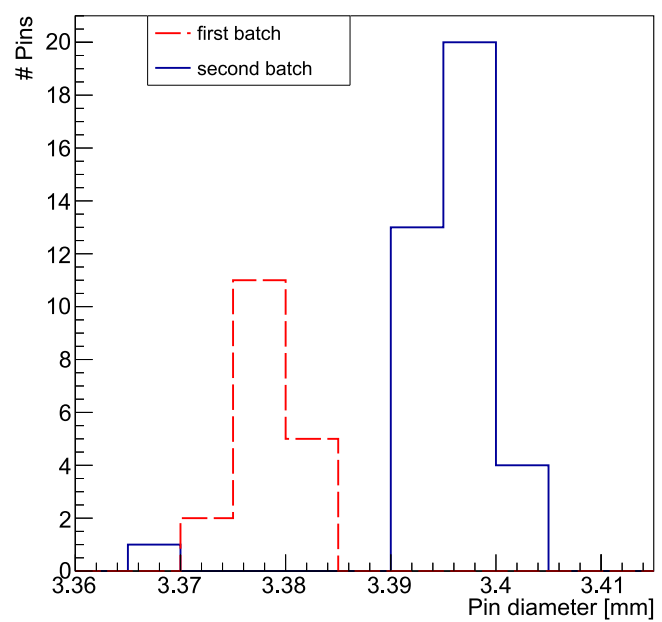

Figure 5.7.: Diameter of the pins for the two batches for the double chip module tools.

The first batch of pins is matched to the first double chip module jig (Plate 0), because it was available to test the pins. The discrepancy between the batches of jigs was not expected. In consequence, the pins have to be adapted to the holes of each batch of jigs. 
This is possible, as the second batch of pins shows, but requires iterative matching of pins and holes. The impact can also be seen comparing the diameter of the quad module jig holes and pins. The pins were produced for a previous jig, and had to be used, because there was not time to produce new ones. The consequences for the alignment precision are discussed later in this section.

The extrusion is the other manufactured part in the jig. It is observed that even if the module is pushed right at the edge, there is still a gap of up to $100 \mu \mathrm{m}$ between them. This influences the position of the flex to the bare module and the tools had to be individually reprocessed to correct for this shift. As this is a systematic effect it can be taken into account for in the design, but to guaranty the tolerances for the alignment the jigs have to be tested individually.

\section{Repeatability of Flex Positioning}

The repeatability of the flex positioning is tested by putting the pins in, placing the flex, measuring the position in $\mathrm{x}$ an $\mathrm{y}$ three times, removing flex and pins, and repeating these steps 50 times. To stabilize the measurement, a dummy silicon piece is used and held by vacuum to have a reference edge without endangering a bare module. The flex is pushed down to flatten it. The jig and pins used are the same with which the double chip modules are built, and the flex SIM2-16, the flex for the last module to be assembled in this batch. Because the building of the modules had higher priority, comparisons between flexes were not possible any more as only this flex was not already glued to a bare module. In Figure 5.8, the average of the three $\mathrm{x}$ and y measurements per repetition are histogrammed. The mean values for $x$ and $y$ direction of $3.21 \mathrm{~mm}$ and $0.70 \mathrm{~mm}$, respectively, are within the spread of both distributions of $10 \mu \mathrm{m}$, exactly at the design values of the tooling of $3.2 \mathrm{~mm}$ and $0.70 \mathrm{~mm}$, respectively. As already seen in the test of the pins and holes where the flex is shifted, a reproducible positioning of the flex on the bare module can be ensured if the pins are fabricated for the holes.

\section{Alignment of Bare Module and Flex}

In contrast to the repeatability studies of the flex position, a double chip bare module is used which allows to measure the critical positioning points instead of the inferred ones, as done with the mentioned study. These points are defined as the distance of the flex to the sensor edge on the side with the flex tail, $x$ distance, and as the distance of the start point of the tail to the closest other edge of the sensor, $y$ distance. In Table 5.3, the measurements of these distances with the flex SIM2-16 and the target values are compared. The result is that each of the jigs fulfils the position requirements within tolerances and statistical uncertainties.

In Table 5.4 and Table 5.5, the corresponding measurement of the assembled quad and double chip modules are summarized, respectively. For the assembly, the one quad chip module jig is used and the double chip module jig with the number 0 for the corresponding modules. The reference point for the double chip module flex is already 


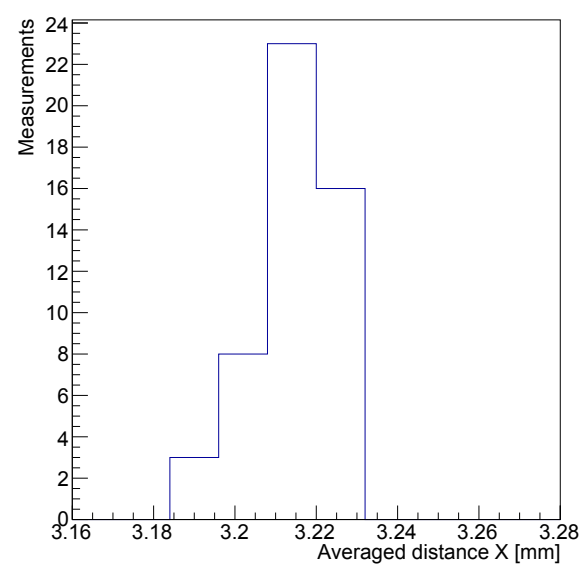

(a)

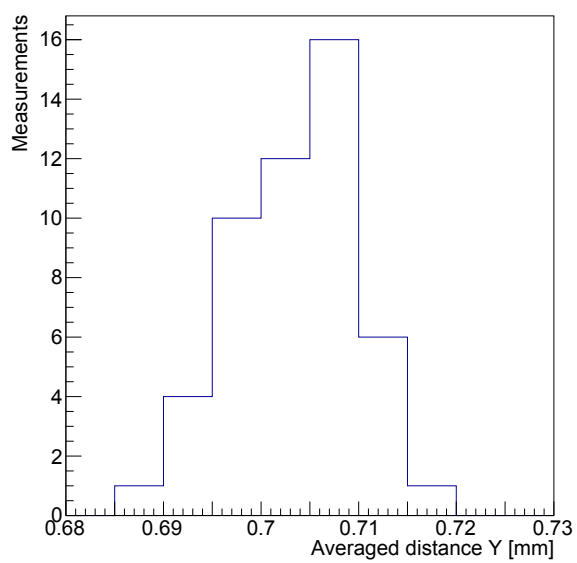

(b)

Figure 5.8.: Distance of read-out chip edge position to flex edge position in $x$ and $y$ direction, design value of the tooling is $3.2 \mathrm{~mm}$ and $0.7 \mathrm{~mm}$, respectively.

described, for the quad chip modules the position is in the centre of the bare module with equal distances to the parallel edges.

For the quad chip modules, there are differences in the alignment values from module to module, which can be explained with the missing matching of the pins to the holes in the jig. Even with this problem the modules, excluding the first one, QSi9, the alignment is within tolerance of $100 \mu \mathrm{m}$. The module QSi9 was the first one to be assembled. The previously described gap between bare module and positioning edges of the jig was at that point in time unknown. In addition, a wrong milling cutter was used producing a slope at the edges, and so an even greater gap opened. This lead to the substantial misalignment and a rework of the jig.

The $x$ alignment for the double chip modules is good, and well in the tolerance.

\begin{tabular}{|l|c|c|}
\hline Jig & $x[\mu \mathrm{m}]$ & $y[\mathrm{~mm}]$ \\
\hline \hline Design & $15(50)$ & $5.573(50)$ \\
\hline Double 0 & $19(6)$ & $5.54(2)$ \\
\hline Double 1 & $6(5)$ & $5.50(3)$ \\
\hline Double 2 & $43(10)$ & $5.51(2)$ \\
\hline Double 3 & $11(8)$ & $5.51(4)$ \\
\hline Double 4 & $30(10)$ & $5.53(4)$ \\
\hline Double 5 & $50(20)$ & $5.51(2)$ \\
\hline
\end{tabular}

Table 5.3.: Test measurements of the alignment points in $x$ and $y$ direction for the double chip module jigs, and the nominal design values. 
Modules DSi13 and DSi14 do not pass the $y$ alignment criterion with 10-20 $\mu \mathrm{m}$ out of the tolerance range. The other modules are well within the tolerance, and match the alignment test of the jig they are built with. Considering the tools and the unknown fabrication tolerances of the flex, this is a good result for the alignment.

\begin{tabular}{|l|c|c|c|c|}
\hline Module & left edge $x[\mathrm{~mm}]$ & right edge $x[\mathrm{~mm}]$ & top edge $y[\mathrm{~mm}]$ & bot edge $y[\mathrm{~mm}]$ \\
\hline \hline Design & 0.25 & 0.25 & 0.075 & 0.075 \\
\hline QSi9 & $-0.50(1)$ & $1.02(1)$ & $-0.28(1)$ & $0.40(1)$ \\
\hline QSi10 & $0.15(5)$ & $0.36(2)$ & $0.08(1)$ & $0.02(1)$ \\
\hline QSi11 & $0.23(2)$ & $0.29(2)$ & $0.13(4)$ & $0.05(2)$ \\
\hline QSi12 & $0.25(1)$ & $0.26(1)$ & $0.10(1)$ & $0.03(1)$ \\
\hline QSi27 & $0.28(2)$ & $0.24(1)$ & $0.14(1)$ & $0.06(2)$ \\
\hline
\end{tabular}

Table 5.4.: Measurements of the alignment points in $x$ and $y$ direction of the assembled quad chip modules, and the nominal design values. Measured at the corners of the module and then averaged.

\begin{tabular}{|l|c|c|}
\hline Module & $x[\mu \mathrm{m}]$ & $y[\mathrm{~mm}]$ \\
\hline \hline Design & $15(50)$ & $5.573(50)$ \\
\hline DSi12 & $20(10)$ & $5.56(1)$ \\
\hline DSi13 & $30(10)$ & $5.51(1)$ \\
\hline DSi14 & $20(10)$ & $5.49(1)$ \\
\hline DSi15 & $40(10)$ & $5.55(1)$ \\
\hline
\end{tabular}

Table 5.5.: Measurement of the alignment points in $x$ and $y$ direction of the assembled double chip modules, and the nominal design values.

\section{Lessons Learned during the Assembly}

During the assembly of the modules, problems are discovered, mistakes made and solutions found. Some of these are summarized here.

A bad handling of the bare module is the easiest way to loose a module right at the start of the assembly. Using proper vacuum tools to pick up the bare module and place it on the jig is the first step. Two sensors broke during this process because the vacuum tweezers had an unreliable way of locking the vacuum. Some kind of pick up tool with even distribution of vacuum over the sensor, and a mechanism to lock the under-pressure would be ideal.

Vias in the flex are on two ways dangerous. First, in the soldering step of the components solder can leak into the vias and produce shorts. Second, the glue applied to the backside of the flex can ooze through to the front side, which during the curing has the plexiglass spacer on top, which then might get stuck on the module. The two times this happened, the spacer could be removed and the modules saved, but only with more force 


\section{Module Assembly and Testing}

then should be applied to the modules. In consequence, a cover layer is added to the latest version of the flex that closes one side of the vias. This simplifies the distribution of glue immensely.

The handling of the tape is difficult, because the tape strips are only about $3 \mathrm{~mm}$ wide and peeling off the cover without damaging the glue requires high dexterity. Also putting the strip manually on the flex is not reliable for positioning. A process in which a liquid glue is spread on the flex in a controlled way is designed for the ITk production and discussed in the next section.

For the wire bonding a stable surface is a key point. If the flex can evade the force used to form the connection with the surface and the wire, the wire will not stick in most cases. As this is a mass production setup, reducing any chance of failure is important. So a good connection of flex and sensor in the region of the wire bond pads has to be ensured with a stable glue layer. The used version of the plexiglass spacer is also designed for protecting the wire bonds later in the assembly process. Therefore, the flex has to be manually pressed down in the wire bond region to connect with the adhesive tape. Later versions of the spacer remove this necessity.

The length and the height of the wire bonds should be minimized, because they are more stable and do not tilt into other wire bonds, potentially creating shorts. For the double chip modules the wires had a length of about $3 \mathrm{~mm}$ due to the constraints on the positioning of the flex, which made manual corrections after the wire bonding to disentangle some of them necessary, which should not be needed. Also the application of the encapsulation of the wires is more difficult if the wires bend easily.

In total five quad chip modules and four double chip modules were assembled for this work. One sensor of each type broke as described above, but still were assembled as first module of their type. Only for this first quad module the alignment is totally wrong, because of the production issues of the first jig. For one read-out chip of a double chip module, a wire bond was misplaced between two read-out chip bond pads, shorting the chip. This could only happen, because the flex foot was ripped off during the movement to the read-out chip pad, there was no tension on the wire and it bent under the bonding tool to the side connecting two pads. With improved parameters for the bonding on the flex, this did not happen again. The remaining modules were assembled without difficulties.

\subsubsection{Outlook on ITk Module Assembly}

The demonstrator is one step on the way to the ITk production. As for the demonstrator not the final ITk components are used, because they are not yet available, the tools will be adapted to the slightly different sizes of the ITkpixV1 read-out chip, or further versions, and the sensors and flexes. This is just a change in position of the pieces relative to each other, but there is a couple of changes that could be made to the design of the tools, which would help the production. Some of these are already incorporated in the design for the RD53A module building tooling.

The whole handling of the flex is at the moment manual. A dedicated jig to position it is designed and will also help the positioning on the bare module. The alignment pins 
for the flex on the jig and the pins for the alignment of the two jigs are disentangled, which allows for optimization of both of them. The jig alignment uses larger diameter rods and ball bearings to reduce position imprecisions, and simplify the handling.

Having the flex in a stable position on a jig, allows for a glue dispensing tool to be placed on top. A stencil with a pattern of holes is foreseen for this, through which the glue then is precisely distributed onto the flex. With this the amount of glue can be controlled, and with the distance of the flex and bare module set by precision screws as distance holders, the thickness of the glue layer and with that the flatness of the overall module can be controlled. This is at the moment not possible with the manual glue application, but a requirement for ITk modules. Also the strength of the adhesion can then be tested systematically with pull tests. Because no dummy silicon and flexes were available, these test were not carried out, as the actual modules are to sparse to do destructive tests with them.

The positioning of the bare module uses the edges of an extrusion, which showed to be feasible but not the most precise production machining step. Also flaws in the edges and the hardness of the aluminium itself might damage the silicon of the bare module, when pushing it against the edges. Instead pins are used as for the flex positioning, and even be made out of or covered with a softer material to reduce the damage potential.

The alignment precision can be improved, as shown above, with a matching of pin and hole diameters. If the pins would be installed in the jig permanently, so not to be removed, the matching uncertainty would be removed, and only the uncertainties on the pin diameter and on the relative position of the pin in the jig would remain. As the tolerances are as low as for the double chip modules, this can increase the precision of the alignment, which is already within tolerances with the current tools, but the margins are not large. Also with the addition of a separate flex jig, more uncertainties might have to be balanced out. In the newest tooling design the pins for the bare module are fixed, but not the pins for the flex due to space restrictions with the glue stencil.

On the topic of quality assessment, the wire bond strength has not been tested until now. A pull tester is available for the wire bond machine, but has to be set up and tested. This will be an important step to qualify the wire bond strength and the overall module assembly process.

After the wire bonding the wires are encapsulated with a silicone elastomer to protect them. This process is at the moment not set up in Göttingen, but carried out at the CERN laboratory of the demonstrator. It is a very delicate step, because no wire can be repaired during or after the coating. For the ITk production this should be qualified at all assembly sites.

An additional step in the production chain for ITk modules will be the high voltage protection of the read-out chips. Because the sensors will be of n-in-p type, the high voltage, up to $800 \mathrm{~V}$ in the later phases of the detector, is on the side towards the readout chips, separated only by the small gap defined by the bump bond thickness. Two methods of coating are proposed, one before assembly, the other after, which will be evaluated, and incorporated in the assembly procedure [21]. 


\section{Module Assembly and Testing}

\subsection{Module Testing}

For the ITk, only modules of high quality in all aspects are allowed to be installed. In this section, the different tests for the ITk modules are briefly introduced, and then some of them are exemplified with results from the assembled modules. In the last part, a prototype of a test stand is described and tested, which combines multiple tests for potentially multiple modules in a controlled environment.

\subsubsection{Test Procedure}

The test procedures described here, focus on testing the quality of the assembled modules and not on the tests of the individual parts before the assembly. The later ones are as important and the comparison from before and after assembly as well, but they are not part of the work presented here.

On reception of the module, a visual inspection is carried out, searching for defects and damage, but also to measure the overall size. The assembly of the module has to fulfil the alignment tolerances of $50 \mu \mathrm{m}$, and in total fit in an envelope defined by the available space at its position in the detector. The measurements of the alignment for the assembled modules are already discussed as part of the production quality in Section 5.1.4.

Then the module is tested for its electrical properties, starting with the sensors currentvoltage (IV) characteristic, functionality and calibration of the read-out chips, and then measurements with particles from radioactive sources or X-ray sources to assess the pixel quality. These tests have to be performed at warm temperature of $20^{\circ} \mathrm{C}$ and at detector operation temperature of $-25^{\circ} \mathrm{C}$ on the module to be sure the module functions as intended in the target environment.

With the last test the mechanical stability of the modules under rapid temperature changes is assessed. In the temperature range of $-55^{\circ} \mathrm{C}$ to $60^{\circ} \mathrm{C}, 100$ temperature cycles are performed with a change rate of $1 \mathrm{~K} / \mathrm{s}$ to check for de-lamination of the flex, and with further radioactive source measurements for bump bond disconnections. The temperature range covers the extreme failure scenarios of $\mathrm{CO}_{2}$ spilling on a module on the one side, and heating up of a module with a cooling failure. In the ITk collaboration these tests are already performed as part of the quality assessment of the module assembly with modules for the demonstrators. The measurements show a large number of disconnected bump bonds at the edges and corners which are the weakest points in the module. It is at least partially contributed to the large copper content in the flex, which drives the difference in thermal expansion coefficient (CTE) between the module parts [20]. In consequence, the design of the flex is adapted and flex materials with lower CTE are investigated. Also the range and the number of cycles are under discussion, as for quality assurance of the assembly process harsher tests are needed, but for the quality control probably not. 


\subsubsection{Electrical Tests}

To connect to the modules, a flex adapter has to be used connecting the connector at the end of the tail of the flex with an adapter card, that breaks out the channels for the different read-out chips, has connectors for low and high voltage, and measurement point for the NTC on the flex. This is not a very secure system, because the flex adapter is very close to the wire bonds for compactness, and connecting and disconnecting the adapter has to be done with great care. The read-out system is the USBPix system using the MMC3 board, controlled via the STControl software [59,60].

Because the cooling setup was not ready at the time of the module assembly and testing, only tests at room temperature are performed for all except one module that was still available when the setup was finished. To remove the heat from the power consumption of the module, it is mounted on the aluminium assembly jig, which also secures the position of the module.

The read-out chips are serially powered with $0.5 \mathrm{~A}$ per chip, so $2 \mathrm{~A}$ or $1 \mathrm{~A}$ depending on quad or double chip module are applied. The resulting analogous and digital voltages per chip can be measured on probe pads on the flex and adjusted with register values to stably generate $1.4 \mathrm{~V}$ and $1.2 \mathrm{~V}$, respectively.

For a precise usage of the charge injection mechanism, the results from the wafer probing of the chips for the injection capacity and the voltage response have to be set in the configuration of the chips in the read-out software.

\section{Sensor Characteristic}

The IV curves of the assembled modules are measured shielded from light, up to a bias voltage of $160 \mathrm{~V}$, so that not for all sensors the breakdown is observed. As reverse biasing is always assumed, the sign of the voltage is neglected. The criteria for a good sensor are a current of less than $1.5 \mu \mathrm{A} / \mathrm{cm}^{2}$ at $V_{\text {depl }}+50 \mathrm{~V}$, and a breakdown voltage greater than $V_{\text {depl }}+80 \mathrm{~V}$ [21]. After bump bonding, the leakage current must not increase by more than a factor of 2 and the breakdown voltage must not change by more than $10 \mathrm{~V}$.

The results are shown in Figure 5.9 for the intact sensors and in the Appendix A.1.1 for the broken sensors. The depletion voltage for all sensors is below $20 \mathrm{~V}$ and the breakdown starts only for two sensors at around $140 \mathrm{~V}$ and the other sensors are stable in the measured range. For the quad chip modules the current is lower than $1.5 \mu \mathrm{A}$, so they fulfil the criterion even without considering the sensor size. The sensor of the double chip modules has a size of $41.315 \mathrm{~mm} \times 19.73 \mathrm{~mm}=8.15 \mathrm{~cm}^{2}$. Dividing the measured current by the area, the double chip modules fulfil the criteria above for good sensors. The differences between the quad and double chip modules can be attributed to the different sensor technology of n-in-p and n-in-n doping. No data is available from the sensor measurements to compare before and after bump bonding.

\section{Read-out Chip Calibration}

A common feature on the read-out chips is that they all have some faulty pixels in the first row, columns 23-30, 35-42, and 47-54. Apparently, this is a result of cutting the 


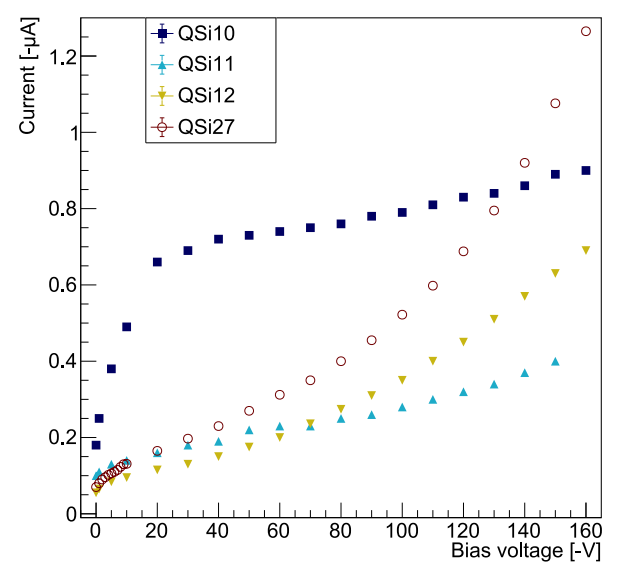

(a)

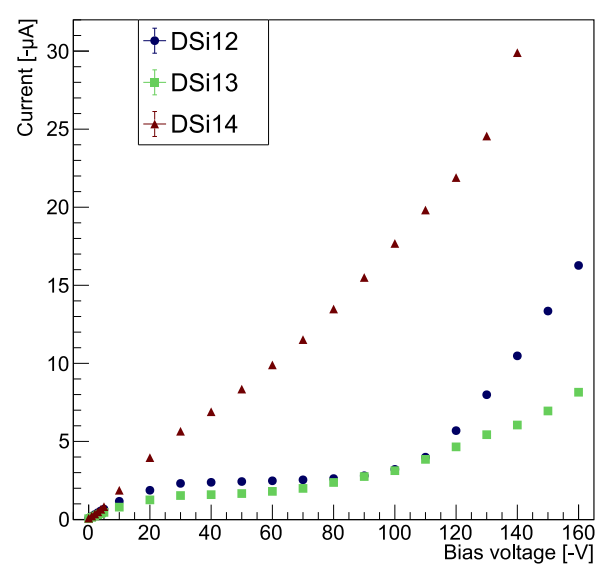

(b)

Figure 5.9.: IV sensor measurements for quad and double chip modules in (a) and (b), respectively.

chips such that they fit to the sensor in the middle region. The top pads are cut off which effects the electronic behaviour of these pixels.

As described in Section 4.4, the FE-I4 chip has a discriminator per pixel, which can be tuned in respect to its threshold and the length of the digitized signal (ToT) corresponding to the charge signal. This suppresses noise and the range and resolution of the collected charge can be set. For the assembled modules standard target tuning values of a 3000 e threshold and a signal of 9 ToT for a signal of 20000 e are used. In Table 5.6 and Table 5.7, the mean values and the standard deviation of the distribution of all pixels for the tuning parameters and the resulting noise in quad and double chip modules are listed, respectively. All functioning read-out chips could be tuned to the target values with the expected spread over the pixel matrix. The means of the noise distributions are in a range of 130-150 e, which is acceptable considering the thickness of the sensor and acceptance ranges set for the IBL production. There are only two special cases with different behaviour. For module QSi10, the pixel in the first two rows of the read-out chips, in the middle of the sensor, have slightly reduced noise of $(115 \pm 10)$ e. Comparing to the results from the measurements with a radioactive source, these rows do not respond to particles and seem not to be connected. Most likely the read-out chips are not in the right position on the sensor. The second case is a double chip module where one read-out chip has lower noise of $(100 \pm 10)$ e. In the source measurements this chip does not see any particles, which could hint at a misalignment of the chip, but position measurements and comparison to functioning modules do not support this. 


\begin{tabular}{|l|c|c|c|c|c|c|}
\hline Module & Threshold $[\mathrm{e}]$ & $\sigma_{\text {thres }}[\mathrm{e}]$ & ToT $[25 \mathrm{~ns}]$ & $\sigma_{\text {ToT }}[25 \mathrm{~ns}]$ & Noise $[\mathrm{e}]$ & $\sigma_{\text {Noise }}[\mathrm{e}]$ \\
\hline \hline QSi9 & 3000 & 40 & 9.03 & 0.08 & 190 & 30 \\
\hline QSi10 & 2640 & 160 & 9.3 & 0.4 & 140 & 10 \\
\hline QSi11 & 3010 & 40 & 9.01 & 0.07 & 140 & 10 \\
\hline QSi12 & 3000 & 40 & 9.03 & 0.07 & 140 & 10 \\
\hline QSi27 (r) & 3010 & 40 & 9.0 & 0.7 & 140 & 10 \\
\hline QSi27 (c) & 2900 & 80 & 8.6 & 0.05 & 120 & 10 \\
\hline
\end{tabular}

Table 5.6.: Results of the tuning of the quad chip modules. The values are the mean values of all pixel in the module and the uncertainties $\sigma$ the standard deviation of the distribution. For the module QSi27 tunings at room temperature (r) and at $-30{ }^{\circ} \mathrm{C}(\mathrm{c})$ are indicated.

\begin{tabular}{|l|c|c|c|c|c|c|}
\hline Module & Threshold $[\mathrm{e}]$ & $\sigma_{\text {thres }}[\mathrm{e}]$ & ToT $[25 \mathrm{~ns}]$ & $\sigma_{\text {ToT }}[25 \mathrm{~ns}]$ & Noise $[\mathrm{e}]$ & $\sigma_{\text {Noise }}[\mathrm{e}]$ \\
\hline \hline DSi12 & 3010 & 40 & 9.1 & 0.3 & 140 & 10 \\
\hline DSi13 & 3010 & 40 & 9.0 & 0.2 & 140 & 10 \\
\hline DSi14 & 3020 & 40 & 9.0 & 0.2 & 130 & 10 \\
\hline DSi15 & 3030 & 70 & 9.0 & 0.3 & 660 & 270 \\
\hline
\end{tabular}

Table 5.7.: Results of the tuning of the double chip modules. The values are the mean values of all pixel in the module and the uncertainties $\sigma$ the standard deviation of the distribution.

\section{Pixel Quality}

To determine the amount of faulty pixels, disconnected pixels here, occupancy measurements with radioactive sources are conducted. In the laboratory an ${ }^{241} \mathrm{Am}$ and a ${ }^{90} \mathrm{Sr}$ source are available, which allow studying the response to photons and electrons, respectively. With the two main photon lines of the ${ }^{241} \mathrm{Am}$ source, the charge response can be calibrated. Most of the measurements are performed with the ${ }^{90} \mathrm{Sr}$ source, because empirically its activity is higher and leads to shorter measurement times to get a fully illuminated module.

As an illustration of how many details can be seen in the occupancy maps, a measurement with the ${ }^{241} \mathrm{Am}$ source for module QSi10 is shown in Figure 5.10 next to a picture of the module. Even individual traces in the flex can be made out, and more obvious the non respondent pixel in the first rows described above.

More occupancy maps for the other modules can be found in Appendix A.1.2. It is interesting to notice that with the quad chip module with broken sensor, QSi9, it is possible to measure particle hits with a bias voltage of $-1 \mathrm{~V}$, while no measurement is possible for the double chip module with broken sensor, DSi15. The n-in-p sensor of the quad chip module already has a small depletion zone at the pixel implant where signal can be collected, but the n-in-n sensor of the double chip module needs the bias voltage.

The number of faulty pixels is determined as the number of pixels with zero occupancy 


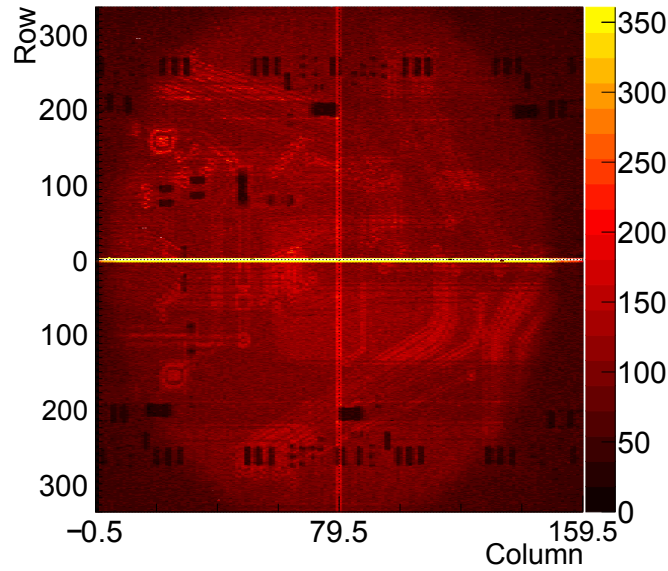

(a)

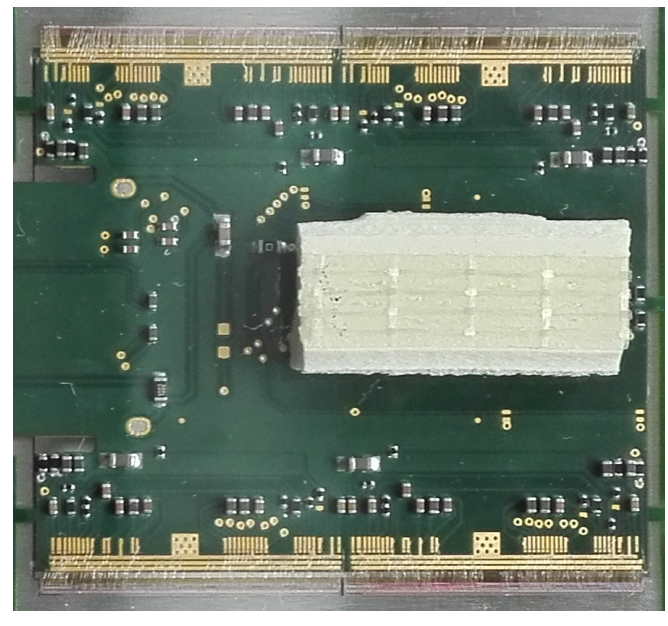

(b)

Figure 5.10.: Occupancy map of an ${ }^{241} \mathrm{Am}$ source measurement for 720 min with module QSi10 in (a) and a picture of the module in transport box in (b) as reference. Measurement done without transport box lid.

and is listed for each module in Table 5.8. A non working pixel rate of less the $4 \times 10^{-3}$ is required for ITk production, which corresponds to less than 107 pixel per FE-I4 chip. For the functioning read-out chips this limit is not reached except for the modules QSi9 and QSi10 with broken sensor and misaligned read-out chips, respectively. Most of the faulty pixels are due to the 21 pixels in the first row of each read-out chip, because of the cutting of the chip.

\begin{tabular}{|l|c|c|}
\hline Module & \# faulty pixels & \# functioning read-out chips \\
\hline \hline QSi9 & 552 & 4 \\
\hline QSi10 & 664 & 4 \\
\hline QSi11 & 74 & 3 \\
\hline QSi12 & 109 & 4 \\
\hline QSi27 & 105 & 4 \\
\hline DSi12 & 53 & 2 \\
\hline DSi13 & 65 & 1 \\
\hline DSi14 & 39 & 1 \\
\hline DSi15 & no test & 2 \\
\hline
\end{tabular}

Table 5.8.: Number of faulty pixels per module, not counting non functioning read-out chips, which are listed separately. 


\subsubsection{Module Test Stand}

For the ITk production, the described tests have to be performed fast, (semi-)automated, and parallelized to ensure the production time line. To achieve this, a test stand is under development bundling the electrical tests at both required temperatures, $20^{\circ} \mathrm{C}$ and $-25^{\circ} \mathrm{C}$. It is housed in a stainless steel box to shield the outside from the radioactive sources, that are used for some of the tests. This limits the available space and requires a compact design of the cooling and read-out systems. In Figure 5.11, a schematic of the distribution of cooling, dry air and cables for a setup with five modules is shown. The dry air is needed to control the humidity in the boxes with the modules, because at low temperatures the dew point can be reached and water condensates and freezes on the modules, which will damage them.

The test stand is developed in parallel to the module assembly and testing activities. Therefore it was not ready to be used for the tests described in the last section.

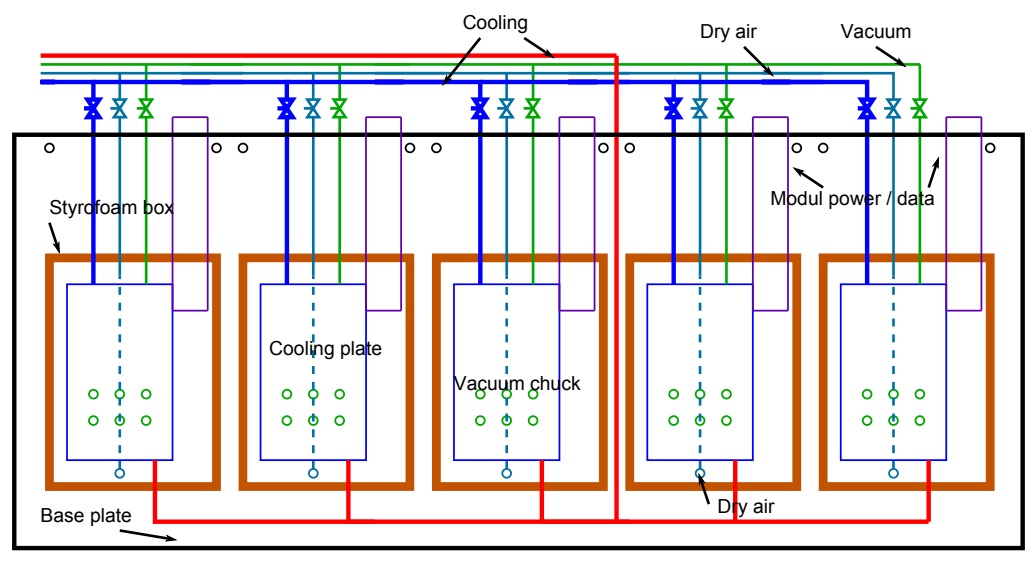

Figure 5.11.: Schematic of a test stand system with cooling (blue), dry air supply (cyan), vacuum supply (green), and read-out and power supply cables (purple) for five modules in their styrofoam boxes (orange).

\section{Cooling System}

As a prototype for the cooling system, a brass plate with a wound path for liquids inside is designed, which is connected to a chiller. On top of the plate, a second brass plate is mounted with a vacuum connection to hold the module in place, alternatively the module can be fixed with screws to the plate. In Figure 5.12, a picture of the prototype can be seen. The modular design allows changing the holder plate in case module dimensions change. The plates are encased in styrofoam and a lid creates a small volume for the module in which the environment can be controlled more easily.

The chiller providing the cooling, is a Huber unistat 705, capable of reaching temperatures of $-70^{\circ} \mathrm{C}$, when using silicon oil as coolant. The pipes are fed through the metal box and care has been taken that at these points the radiation shielding is ensured. 


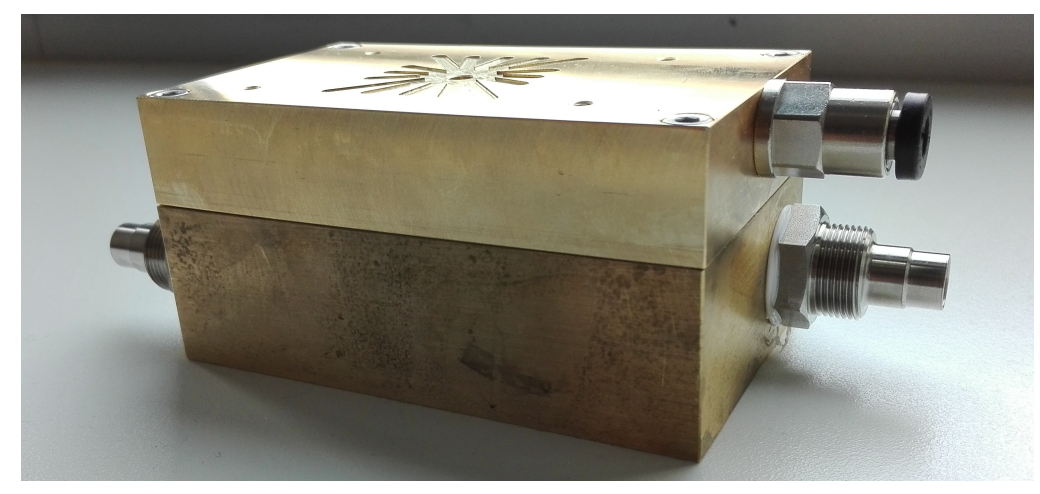

Figure 5.12.: Prototype of the cooling plate and holder on top.

In the styrofoam box four Pt1000 temperature sensors are installed to check the system at different points: one at the cooling plate, one at the vacuum plate, one dangling from the lid for the air temperature, and one on the aluminium transport plate on which the module is placed. In Figure 5.13, a picture of the setup with indications to the sensors is shown. As mentioned, the module is mounted in the setup by screwing its transport plate to the holder and applying vacuum for a good thermal contact. The threads in the holder and the transport plate do not necessarily match, so that a gap is left between the two. Applying vacuum then only draws in wet air into the styrofoam box, which is counter productive. A first solution is to remove the thread from the transport plate, which allows closing the gap, but the mounting procedure is more unstable as the module is for a short time not fixed to the plate. Further improvements like a better transport plate will be necessary to ensure no accidents can happen.

The setup is completed with a humidity sensor HIH4000 for the relative humidity RH, in combination with the air temperature $T_{\mathrm{amb}}$, the dew point is calculated [61]:

$$
T_{\mathrm{dp}}=\frac{c \cdot\left(\ln \left(\frac{\mathrm{RH}}{100}\right)+\frac{b \cdot T_{\mathrm{amb}}}{c+T_{\mathrm{amb}}}\right)}{b-\left(\ln \left(\frac{\mathrm{RH}}{100}\right)+\frac{b \cdot T_{\mathrm{amb}}}{c+T_{\mathrm{amb}}}\right)},
$$

with the values $c=241.2^{\circ} \mathrm{C}$ and $b=17.5042$.

The temperature and humidity sensors are read and recorded with a data logger, dataTaker DT80, in intervals of $10 \mathrm{~s}$. In Figure 5.14, the measurements of all sensors or derived quantities are displayed. These measurements were taken during a cool down to $-30{ }^{\circ} \mathrm{C}$ on the module QSi27, and then warm up to room temperature. The temperature changes are very smooth and at no point in time the dew point is reached, because the air is dry enough from the dry air flow, which is also pre-cooled to not flush the volume with room temperature air. Because the coolant has to be passed into the metal box, the insulation at these points is not optimal and cooling power is lost. Therefore the cooling plate and the holder are not as cold as the liquid at the chiller exit. A small loss can also be seen from the cooling plate to the transport plate of the module. The vacuum holding the transport plate does not improve the thermal contact, but worsens 


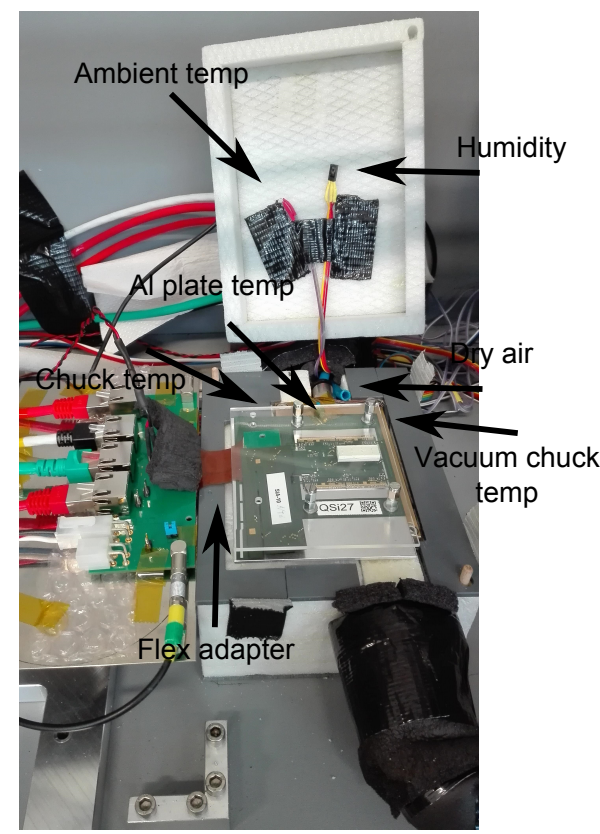

Figure 5.13.: Picture of the cooling setup in the metal box with indicators for the location of the different sensors. The module QSi27 is mounted.

it, as can be seen when the vacuum is turned off. The largest heat source in the system is the module itself, as the temperature jump of $9 \pm 0.3^{\circ} \mathrm{C}$ shows, when turning the module off at cold temperature.

This test shows that the prototype cooling system can reach temperatures below the required value and in a timely fashion of half an hour cool time. Also, the electrical tests can be performed in the current setup, see next section. For the future ITK quality control tests, the setup needs to be scaled to multiple modules to allow a sufficiently fast throughput. Subsequently, the cooling performance must be evaluated under a higher load.

\section{Motorised Source Holder}

One of the electrical tests is a measurement with a radioactive source. If multiple modules are tested in parallel, the source has to be moved between these modules. Any manual intervention slows down the process, and from a radiation safety point of view should be avoided. Therefore a motorised holder for the source has been developed in the context of a bachelor thesis [62].

In Figure 5.15, the holder on the two axis system can be seen. A program controls the movement of the holder in the $\mathrm{x}$ and $\mathrm{y}$ plane by sending commands to an arduino, which communicates with the motors. The precision of the positioning is determined to $1 \mathrm{~mm}$, which is an order of magnitude better than needed to position the radioactive source above a module. The system can be moved manually up and down by mounting 


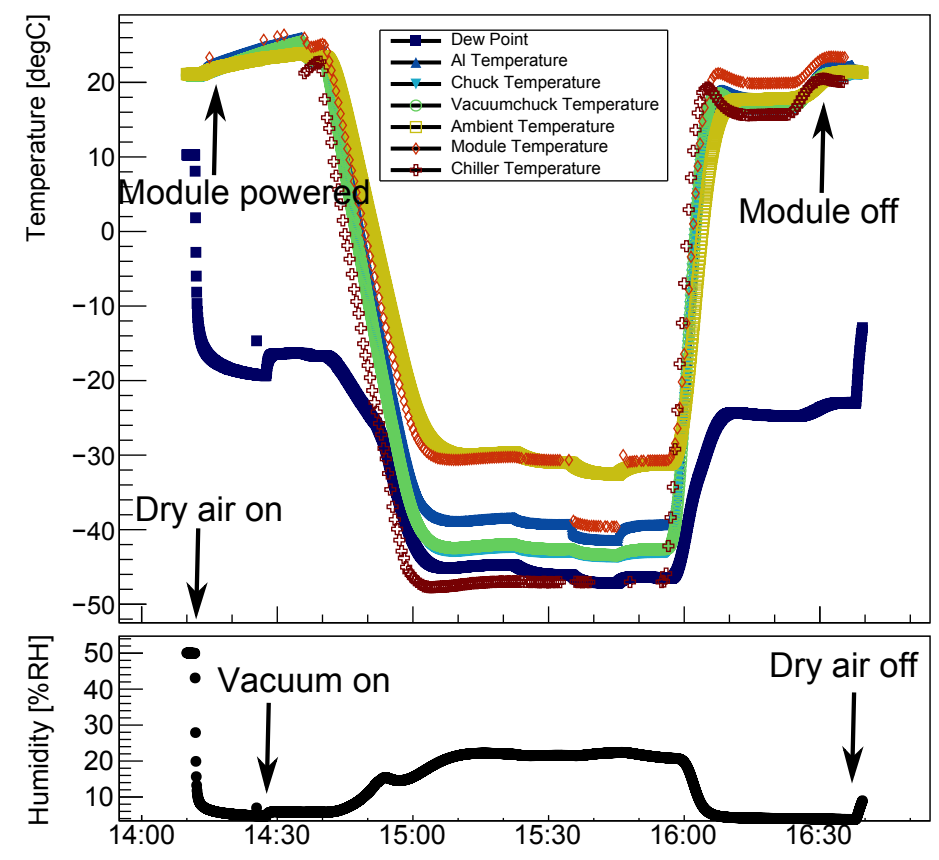

Figure 5.14.: Cool down and warm up cycle in the prototype test stand with the module QSi27. More detailed views in Appendix A.1.3.

it in different positions. As the system is quite heavy, this is only foreseen to find an optimal distance of source to module.

The source can be blocked with a lead block that is controlled with a magnetic solenoid actuator and set back with a spring. An interlock system is in place to stop movement of the holder and block the source if the metal box doors are opened.

The control of the motors is also implemented as an addition to the STControl software. Also, for multiple modules a communication tool exists, to ensure the source is moved to the requesting module after the previous modules source measurements are done.

An occupancy map from a measurement with the ${ }^{241} \mathrm{Am}$ source and the module QSi27 at $-30^{\circ} \mathrm{C}$ is shown in Figure 5.16(a). No significant differences to previous measurements with the module are detected. Because the plexiglass lid of the transport box is on to protect the module, the features of the flex are not as clear and a part on the right side is blocked additionally by the foam used to hold the module down in the transport box.

In Figure 5.16(b), the hit rate of at different positions of the ${ }^{241} \mathrm{Am}$ source in the test stand measured with the module QSi27 is shown. The hit rate is maximal at the position of the module at $290-310 \mathrm{~mm}$ in $x$ position and around $80 \mathrm{~mm}$ in $y$ position of the source. The next modules will be placed in a distance of $100 \mathrm{~mm}$ in $x$ direction, to fit 


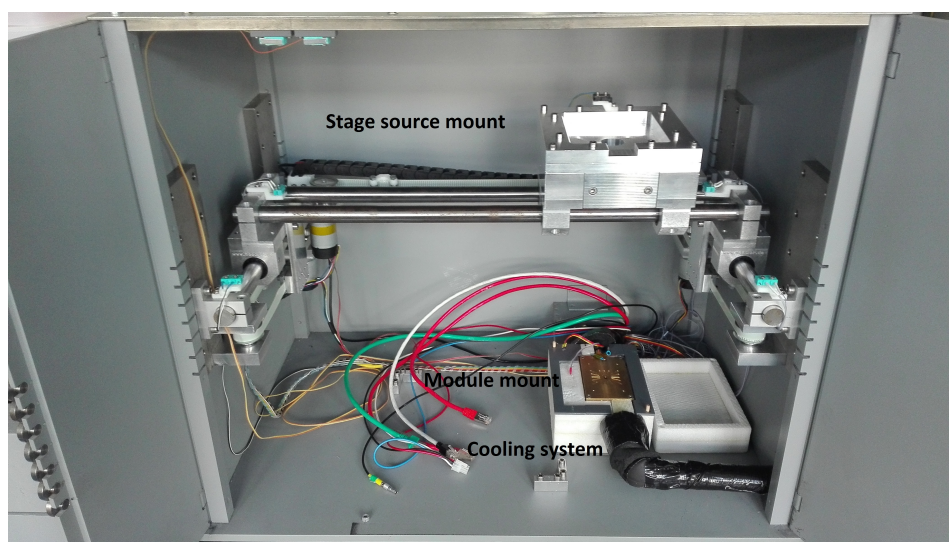

Figure 5.15.: Picture of the motorised source holder in the test stand.

as many modules as possible in the test stand. At this position the hit rate is negligible, which ensures that the measurements at neighbouring module spots are not disturbed.

This test concludes the status of the prototype test stand. It is operational for one module at the time in the required temperature range. The prototype will be adapted to the final ITk module dimensions and the read-out system with the dedicated cabling. The remaining space in the metal box will be filled with spots for modules. 
5. Module Assembly and Testing

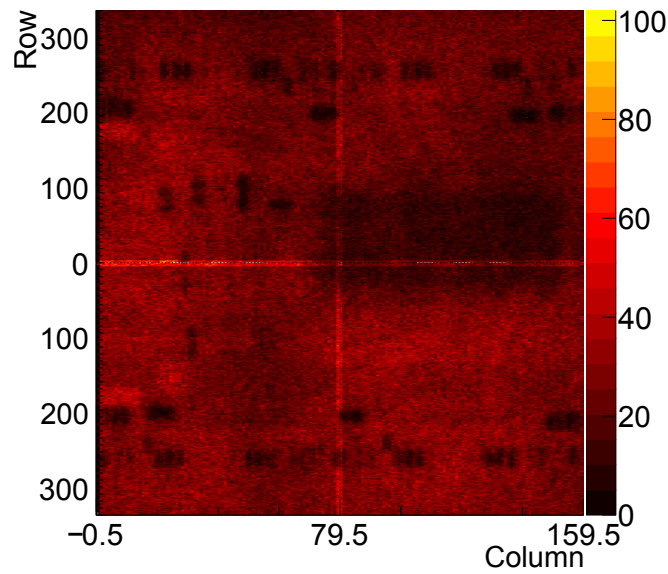

(a)

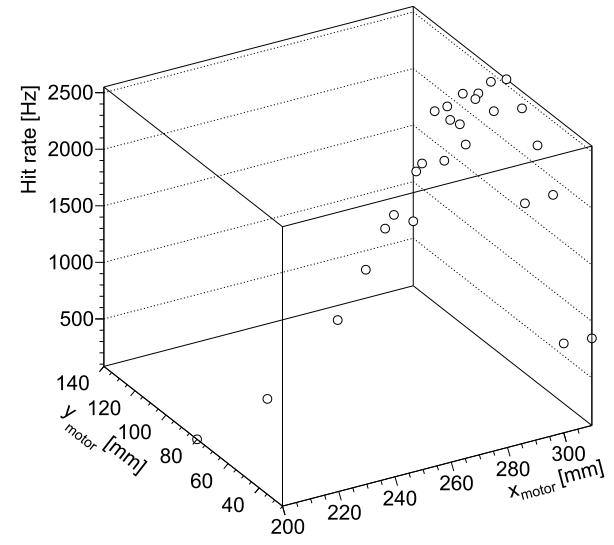

(b)

Figure 5.16.: (a): occupancy map for a measurement with the ${ }^{241} \mathrm{Am}$ source and the module QSi27 at $-30{ }^{\circ} \mathrm{C}$. (b): hit rate at different positions of the ${ }^{241} \mathrm{Am}$ source in the test stand measured with the module QSi27. 


\section{CHAPTER 6}

\section{Test Beam Measurements with Diamond Pixel Modules}

In the RD42 Collaboration [63], diamond is studied as a sensor material for particle physics, and one focus is the development of a pixelated 3D diamond detector. In the context of this thesis, two diamond sensors are bump bonded to ATLAS FE-I4 read-out chips: one of them with planar electrode geometry, the other with 3D electrodes in the bulk. Tests are performed in the laboratory in Göttingen before test beam measurements are conducted in two campaigns at the Deutsches Elektronen-Synchrotron (DESY) in Hamburg.

In this chapter, first the experimental setup at DESY and the diamond detectors under test (DUTs) are described in Section 6.1. Then the analysis software and strategy to interpret the measured data is presented in Section 6.2. Finally, in Section 6.3, the results of the measurements are reported and discussed.

\subsection{Experimental Setup}

\subsubsection{Test Beam Infrastructure}

There are three main parts needed at a test beam facility: the beam, the telescope and the data acquisition hard- and software.

\section{Particle Beams}

The electron or positron beams used as the test beams are generated starting from the DESY-II synchrotron [64]. A bremsstrahlung beam is generated from the electrons or positrons in the machine hitting a carbon fibre target. The photons are converted again at a metal plate into electron and positron pairs. From these the final beam is formed by selecting the particle energy by deflection with a dipole magnet into a collimator, which blocks part of the beam. In Figure 6.1, a schematic of the described processes at 


\section{Test Beam Measurements with Diamond Pixel Modules}

the area is depicted. In total there are four beam areas, where experiments can be set up and conducted. For the work in this thesis the areas T21 and T22 were used.

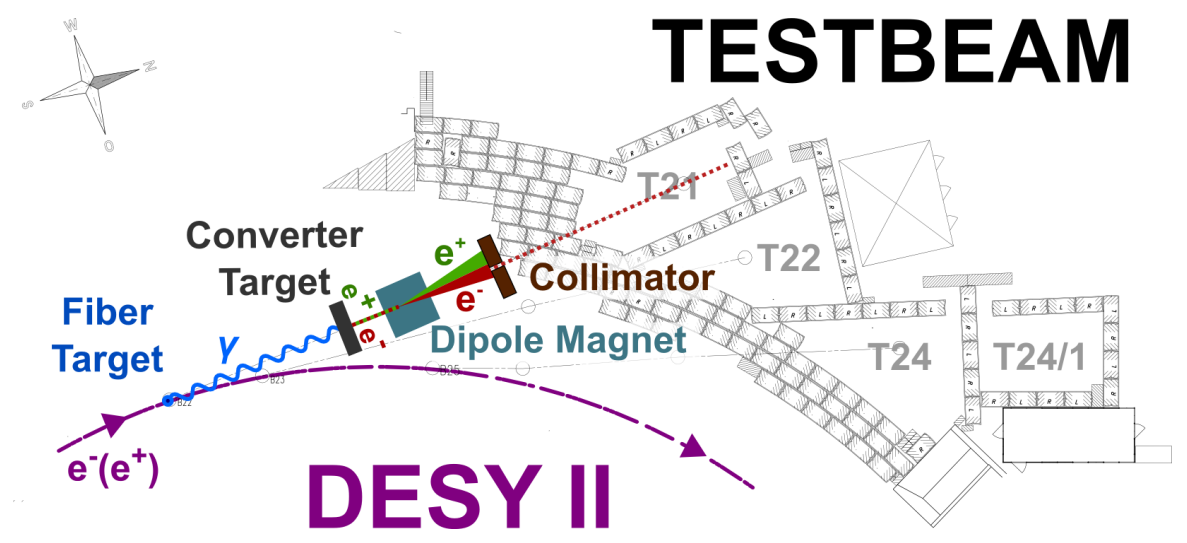

Figure 6.1.: Schematic of the test beam area at DESY [64].

The beam energy is limited by the DESY-II electron or positron energy of $6 \mathrm{GeV} / \mathrm{c}$. Typical beam energies chosen for the measurements are $3-5 \mathrm{GeV} / \mathrm{c}$, because the particle rate decreases with higher energy, but the multiple scattering increases with lower energy ${ }^{1}$.

\section{Telescope}

The beam is tracked with a so called telescope to allow position resolved measurements of the DUT. In case of the setup at DESY, this consists of six detector planes, which are aligned in beam direction, see a picture in Figure 6.2 [65]. The detector in each plane is based on the MIMOSA 26 silicon sensor with quadratic pixels with a pitch of $18.4 \mu \mathrm{m}$ [66]. It is read out in a rolling shutter mode with an integration time of $115.2 \mu \mathrm{s}$. They are mounted in aluminium jigs for stability and connection to a cooling system, with the active area of the detectors cut out in the jigs and only protected from light by a thin Kapton foil. This minimises the material in the beam and reduces therefore the multiple scattering, which is important at these comparatively low beam energies.

The telescope planes are organized in two pairs of three, leaving space in between the upstream and downstream arm for the placement of the DUTs. For this a micrometer table is provided to align the DUTs precisely in the beam. The $\mathrm{z}$ axis of the right-handed coordinate system points in beam direction and the $\mathrm{x}$ and $\mathrm{y}$ axis are in the plane of the detectors. Following the beam the planes are numbered starting with zero at the first plane in the beam, which is also the origin of the global coordinate system. For each detector, there is a local coordinate system defined with the axis $(w, u, v)$. The origin is in the middle of the sensitive area of the detector. The $u$ and $v$ axis define the plane of the detector, pointing in the direction of rising column and row numbers, respectively. Perpendicular to this, the $w$ axis is defined. A transformation between global and local

\footnotetext{
${ }^{1}$ In the following natural units are used for $c$ and the beam energy, setting $c=1$.
} 


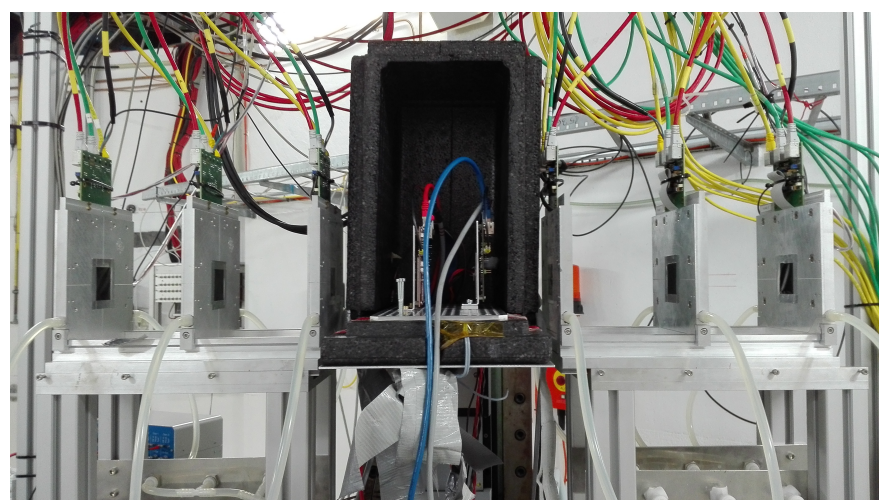

Figure 6.2.: Picture of the telescope DATURA in test beam area 21 with reference sample and DUT mounted between the two telescope arms in a styrofoam box for light protection.

coordinate system can be defined by simple translation and rotation of the axes. The associated angles of the rotation of the axes $w, u$, and $v$ are named $\gamma, \alpha$, and $\beta$.

\section{Data Acquisition}

Two crossed scintillators are mounted at each side of the telescope, defining a read-out area of $20 \mathrm{~mm} \times 10 \mathrm{~mm}$ and providing a fast trigger signal to the Trigger Logic Unit (TLU) [67]. With coincidence of the scintillators, the trigger signal is distributed by the TLU to the read-out systems of the telescope plane and the data acquisition (DAQ) systems used for the DUTs in this case the USBPix system [59]. Trigger numbers for later synchronisation of the data are sent to all of the DAQ systems, and vetos can be received from them to disable the triggering. The EUDAQ framework is used to integrate the different DAQ systems [68]. The communication is done by TCPIP protocol, and the data is synchronised to global events from the data of the individual systems. A graphical user interface is provided to connect and control the different systems, and start and stop the data taking. Additionally, a logging collector and an online monitor for on the fly quality checks of the data are available.

\subsubsection{Diamond Pixel Modules}

The DUTs are hybrid pixel modules consisting of the diamond sensor bump bonded to an ATLAS FE-I4 read-out chip, and are mounted on a rigid PCB for better handling. The sensors are polycrystalline diamonds with a size of $(5 \times 5) \mathrm{mm}^{2}$ and thickness of $500 \mu \mathrm{m}$. They have a CCD of $260 \mu \mathrm{m}$ at high electrical fields [69]. 3D electrodes are fabricated in one of the sensors at Oxford, the other one has a planar electrode geometry. The 3D electrodes have a diameter of $6 \mu \mathrm{m}$. The metallisation on both of them is similar, because it is designed for the 3D diamond to connect the electrodes to the bump bond locations or the bias voltage, but was tested first on a diamond without bulk electrodes. 


\section{Test Beam Measurements with Diamond Pixel Modules}

With the planar diamond, the bump bonding to the FE-I4 was first established at IFAE Barcelona as a test for the 3D diamond.

In Figure 6.3(a), a microscope picture with $50 \times$ magnification of the middle area of the $3 \mathrm{D}$ diamond is shown. The metallisation is placed on both sides of the diamond in the centre with a size of $(3.0 \times 3.25) \mathrm{mm}^{2}$. The top side distributes the bias voltage with traces in a grid of rectangles and hexagons to the field creating electrodes in the bulk forming rectangular and hexagonal pixels. The rectangular pixels have a size of $(125 \times 100) \mu^{2}$ and the hexagons have a pitch in column direction of $113.8 \mu \mathrm{m}$ and a maximal extension in row direction of $133.3 \mu \mathrm{m}$. Due to defects in the diamond and wrongly fabricated electrodes, not all electrodes are connected to the bias grid to prevent shorts through the diamond to the read-out chip. The charge collecting electrodes are connected to the read-out chip with small metal traces on the surface of the diamond. A layout of the pixel matrix can be found in Figure 6.3(b). The disconnected electrodes are not represented in the layout for simplicity. The non metallised area of the diamond is divided into $(250 \times 50) \mu^{2}$ sized pixel, because the bump bonds in this area connect the bare diamond to the read-out chip.

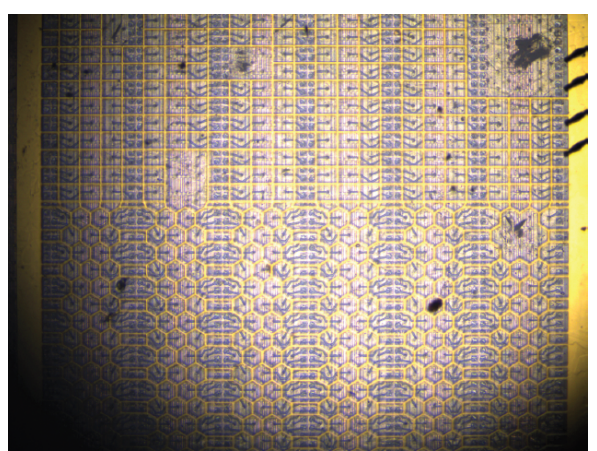

(a)

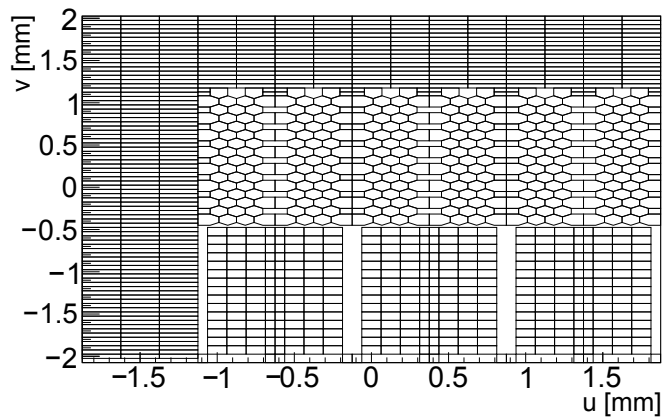

(b)

Figure 6.3.: (a): picture with $50 \times$ magnification of the middle area of the 3D diamond. (b): representation of the pixel matrix of the $3 \mathrm{D}$ diamond not considering not connected electrodes.

The diamond is positioned on the read-out chip in the top right corner with the first electrodes on the edge of the chip. The connected read-out pixels are listed in Table 6.1. A part of the diamond is overhanging over the edge of the read-out chip.

\begin{tabular}{|c|c|c|c|c|c|c|}
\hline \multirow{2}{*}{ Module } & \multicolumn{2}{|c|}{ Diamond area } & \multicolumn{2}{c|}{ Rectangular pixel } & \multicolumn{2}{c|}{ Hexagonal pixel } \\
& Column & Row & Column & Row & Column & Row \\
\hline Planar diamond & $61-78$ & $0-92$ & $64-76$ & $13-42$ & $64-76$ & $43-75$ \\
\hline 3D diamond & $65-79$ & $0-80$ & $68-79$ & $0-30$ & $68-79$ & $31-64$ \\
\hline
\end{tabular}

Table 6.1.: Position of the diamonds on the read-out chip. 
As the planar diamond is a test fabrication for the metallisation and the bump bonding, the metallisation is very similar, without the changes due to the defects, see a picture in Figure 6.4(a). Because no electrodes are in the bulk, the trace metallisation acts as pixelation of the sensor. The layout of the traces is shown in Figure 6.4(b). The different regions are named as for the 3D diamond, rectangular and hexagonal pixel areas, although no such shaped pixels are present, because the metallisation is essentially the same but without the bulk electrodes. In contrast to the 3D diamond, the planar one is fully placed on the read-out chip, still in the top right corner. The list of connected pixels is also in Table 6.1.

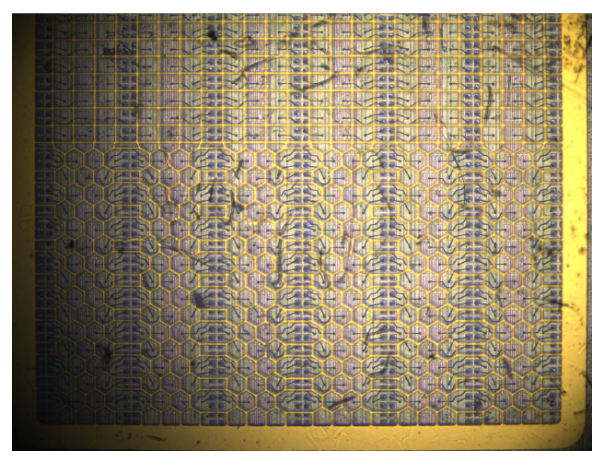

(a)

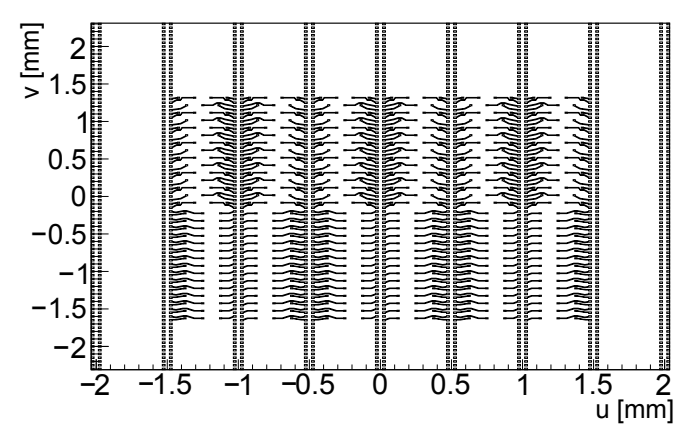

(b)

Figure 6.4.: (a): picture with $50 \times$ magnification of the middle area of the planar diamond. (b): layout of the traces forming the pixels of the planar diamond.

The IV curves of the diamond modules are displayed in Figure 6.5. While the planar diamond draws almost no current, the 3D diamond has a threshold at about $55 \mathrm{~V}$ after which the current increases drastically. An earlier breakdown is expected because of the shorter electrode spacing in the 3D diamond. This limits the operation range of that diamond to around $70 \mathrm{~V}$ in order not to damage the read-out chip. The electrical field in the 3D diamond is about $20 \%$ lower for a bias voltage of $55 \mathrm{~V}$ than in the planar diamond at $500 \mathrm{~V}$ considering only the distances of the electrodes.

The read-out chips are tuned to a threshold of $1500 \mathrm{e}$ and a charge response of 7 ToT for an injected charge of $14 \mathrm{ke}$ to be able to detect the expected small signals from the diamond. In $500 \mu \mathrm{m}$ thick diamond a signal of $18 \mathrm{ke}$ is generated by a particle not considering trapping effects. This is taken into account for the charge response tune. The tuning results are listed in Table 6.2. While the read-out chip for the planar diamond has a higher base noise in all the pixels, because it is not a good quality chip, there are no remarkable pixels in the diamond area. For the $3 \mathrm{D}$ diamond this is different, as a sizeable amount of pixels do not seem to be tunable in the diamond area and are in later tests disabled. The rest has the same characteristics as the pixels not connected to the diamond.

To test the quality of the modules, a ${ }^{90} \mathrm{Sr}$ source is used to measure the response to particles, electrons in this case. In Figure 6.6, exemplary hit maps of the two modules 


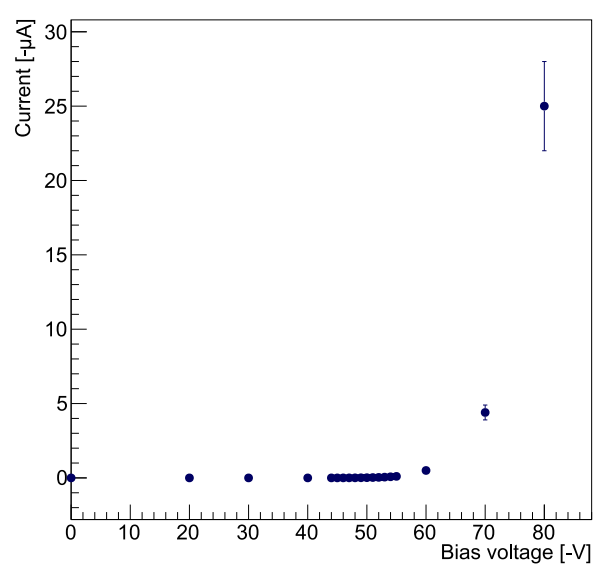

(a)

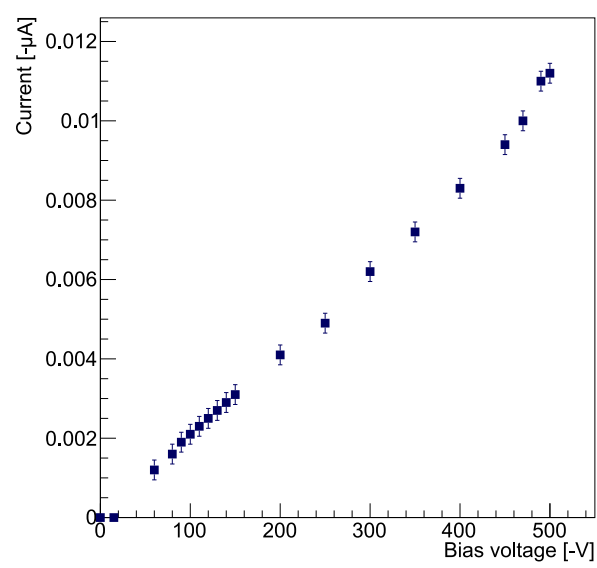

(b)

Figure 6.5.: IV characteristic of the 3D (a) and the planar diamond module (b). Note the different axes ranges.

\begin{tabular}{|l|c|c|c|c|}
\hline Module & Usage & Threshold $(\sigma)[\mathrm{e}]$ & Noise $(\sigma)[\mathrm{e}]$ & ToT at $14 \mathrm{ke}(\sigma)[25 \mathrm{~ns}]$ \\
\hline \hline Planar & Lab & $1540(120)$ & $310(20)$ & $7.3(2)$ \\
\hline Planar & TB & $1560(140)$ & $310(25)$ & $7.3(2)$ \\
\hline 3D & Lab & $1470(40)$ & $150(20)$ & $6.9(5)$ \\
\hline 3D & TB & $3230(70)$ & $105(10)$ & $6.9(5)$ \\
\hline
\end{tabular}

Table 6.2.: Results of the tuning, mean and standard deviation of the distributions for all read-out chip pixels of the diamond modules, used tunings for the test beam measurements (TB) are indicated, the others are used in laboratory measurements (Lab).

are displayed. To be able to measure particle signals and not noisy pixels, about $25 \%$ of the 3D diamond pixels have to be masked in contrast to a handful for the planar one, see maps in Figure 6.7. Particles are measured with both devices, but for the planar diamond with a higher rate of $10 \mathrm{~Hz}$ compared to less than $1 \mathrm{~Hz}$. Also, a difference can be made out between the metallised area and the rest of the diamond as in the latter one significantly less hits are registered in the planar diamond. Because of the noisy pixels in the 3D diamond, a tuning of the threshold to $3000 \mathrm{e}$ is performed, which does not reduce the noisy pixel count, see result in Table 6.2. To be on the safe side concerning the noise this tuning is used in the test beam measurements for the $3 \mathrm{D}$ diamond. 


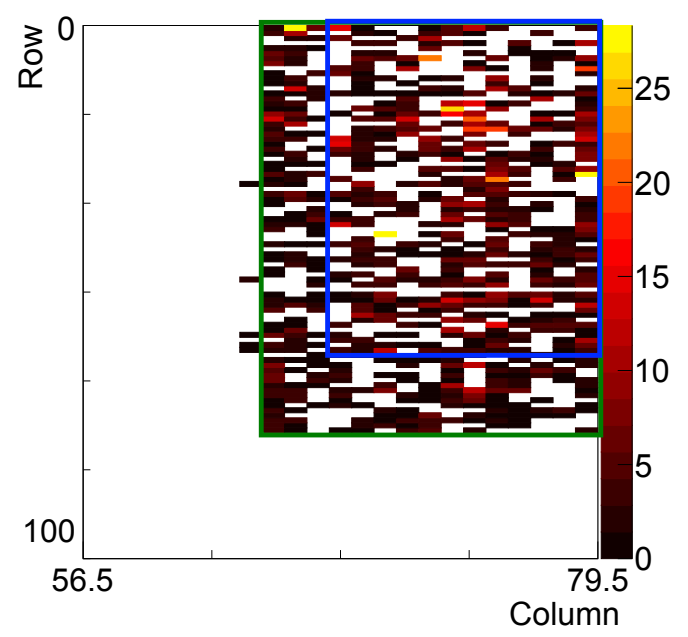

(a)

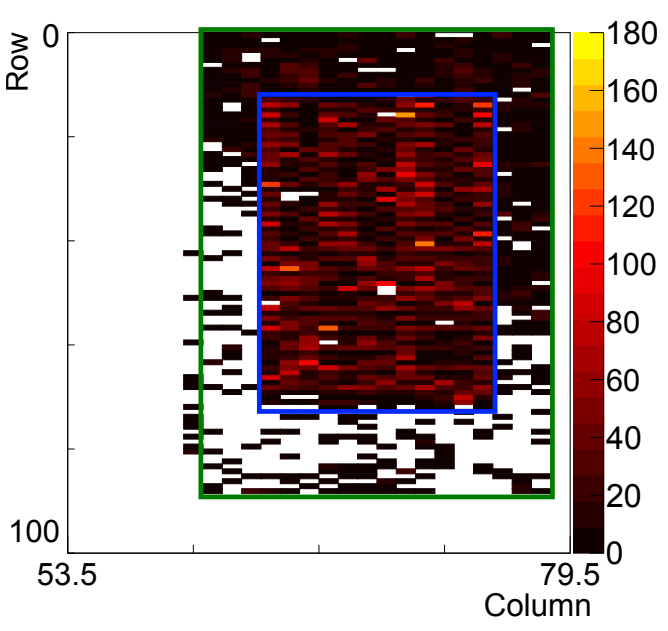

(b)

Figure 6.6.: (a): hit map per pixel for 3000 hits from a ${ }^{90} \mathrm{Sr}$ source in the $3 \mathrm{D}$ diamond tuned to a threshold of $1.5 \mathrm{ke}$ at $60 \mathrm{~V}$ bias voltage. (b): $20 \mathrm{k}$ hits in the planar diamond at a bias voltage of $350 \mathrm{~V}$. Diamond area marked in green and metallised area in blue.

\subsubsection{Measurements at DESY}

The data from the DUTs are acquired with the USBPix system using the MIO3 and MMC3 board and the STControl software, which is interfaced into EUDAQ [59, 70]. Because of the long integration time of the read-out of the MIMOSA 26 sensors compared to the ATLAS FE-I4 operating at $40 \mathrm{MHz}$, an additional FE-I4 reference sample is used to provide a time stamp for the reconstruction of the events.

\section{Reference Module}

The reference sample used is a planar silicon module ${ }^{2}$. It has a silicon n-in-n sensor of $100 \mu \mathrm{m}$ thickness bump bonded to a FE-I4 read-out chip. Around the edges a lot of pixels are noisy probably due to a faulty under bump metallisation. Because the diamond modules have a small size, the centre region of the module is enough as a reference area and accordingly arranged in the beam. The noisy pixels are determined with an analogue scan, in which multiple times charges are injected into the pixels. Noisy pixels report more hits than injections and are then disabled for the measurements. The read-out chip is tuned to a threshold of $1500 \mathrm{e}$ and a charge response of $7 \mathrm{ToT}$ for a signal of $14 \mathrm{ke}$. With a bias voltage of $80 \mathrm{~V}$, it is operated fully depleted, as the depletion voltage was previously measured to be around $10 \mathrm{~V}$ [71].

\footnotetext{
${ }^{2}$ Identifier: ADVACAM NP-100-5-3B
} 


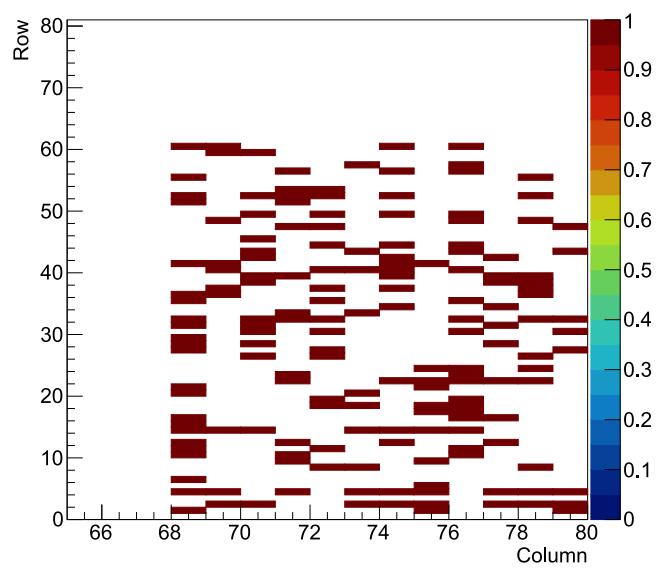

(a)

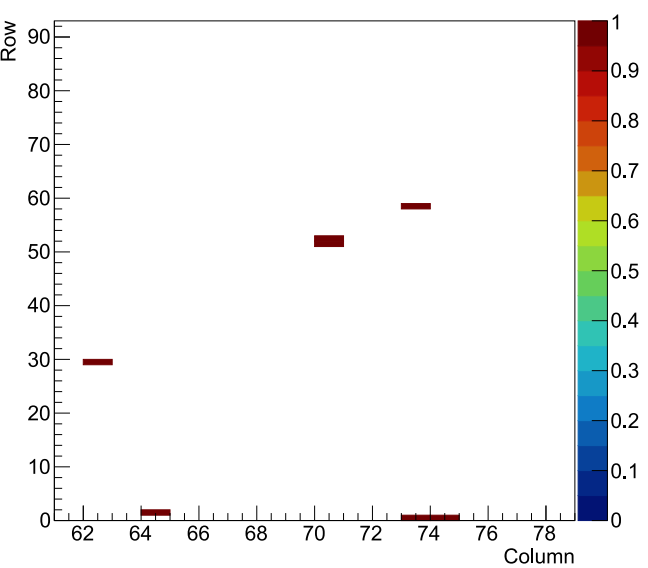

(b)

Figure 6.7.: Entries mark disabled pixels for source and test beam measurements for the $3 \mathrm{D}$ and planar diamond module in (a) and (b), respectively.

\section{Measurements}

Measurements were conducted in two test beam periods, once with the planar diamond module in test beam area T21 and the second with the 3D diamond module in area T22. Each of the areas has its own telescope, the DATURA in area T21 and the DURANTA in area T22. The performance has been reported as similar [65].

For each detector the dependence of the performance on the bias voltage is studied. In Table 6.3 and Table 6.4, the different settings and the recorded number of events are listed for the planar and the 3D diamond module, respectively. A special data set is recorded for the $3 \mathrm{D}$ diamond with an inclination of $15^{\circ}$ of the module to the beam to test the influence of the inactive material of the bulk electrodes.

Distances between telescope planes for the measurement periods can be found in the appendix in Table A.1. The measurements of the planar diamond module were split into two time slots. In between the telescope setup was slightly changed.

Because of not yet completely understood interplay between the DAQ for the DUT, probably the MIO3 board and the TLU, the synchronisation of the telescope and the DUT was often lost during the measurements with the planar diamond. Later, the synchronisation was recovered with an extra script ordering the events offline ${ }^{3}$. For the measurements with the 3D diamond detector, the MMC3 board is used and no synchronisation problems observed.

\footnotetext{
${ }^{3}$ Botho Paschen: https://github.com/BothoP/eudaq/tree/feature/Helge-san
} 


\begin{tabular}{|c|c|c|c|}
\hline Bias voltage $[-\mathrm{V}]$ & Beam energy $[\mathrm{GeV}]$ & Batch & \# Events [Mio] \\
\hline \hline 50 & 4.6 & 2 & 1.6 \\
\hline 100 & 4.6 & 2 & 3.5 \\
\hline 150 & 4.0 & 4 & 2.2 \\
\hline 200 & 4.6 & 2 & 4.9 \\
\hline 250 & 4.6 & 2 & 6.2 \\
\hline 300 & 4.6 & 2 & 5.5 \\
\hline 350 & 4.6 & 2 & 7.6 \\
\hline 400 & 4.6 & 2 & 17.5 \\
\hline 500 & 4.6 & 2 & 6.1 \\
\hline 600 & 4.0 & 4 & 2.6 \\
\hline 600 & 4.6 & 4 & 1.5 \\
\hline
\end{tabular}

Table 6.3.: Collected data sets with the planar diamond module. Batch denotes the different telescope setups.

\begin{tabular}{|c|c|c|c|}
\hline Bias voltage $[-\mathrm{V}]$ & Beam energy $[\mathrm{GeV}]$ & Inclination $\left[^{\circ}\right]$ & \# Events [Mio] \\
\hline \hline 50 & 4.0 & 0 & 2.9 \\
\hline 60 & 4.0 & 0 & 12.1 \\
\hline 60 & 4.0 & 15 & 2.0 \\
\hline 70 & 4.0 & 0 & 1.5 \\
\hline
\end{tabular}

Table 6.4.: Collected data sets with the 3D diamond module.

\subsection{Analysis Strategy}

The processing of the test beam data is performed in several steps: the telescope setup is calibrated, the particle tracks reconstructed, and the DUT and track data compared. The analysis software used for this is introduced in Section 6.2.1, and upgrades of the software implemented for this analysis are presented. Then the different steps for the analysis of the test beam data are described in Section 6.2.2.

\subsubsection{Analysis Software}

The measured test beam data are analysed with the Test Beam Software Framework $(\mathrm{TBSW})^{4}$. It is based on Marlin (Modular Analysis \& Reconstruction for the Linear Collider) processors, which are $\mathrm{C}++$ classes that are set up to provide the different steps to calibrate and reconstruct the data. The analysis is done event by event, handed down a chain of processors, reading the data, and writing new data as output. LCIO (Linear Collider IO) files are used to guarantee the consistency of the data throughout the processor chain. The raw data events are read in with the integrated EUDAQ

\footnotetext{
${ }^{4}$ https://bitbucket.org/testbeam/tbsw
} 


\section{Test Beam Measurements with Diamond Pixel Modules}

framework. The parameters of the processors are set and their chain constructed by python scripts. For the final output TTrees from the ROOT analysis package are used, storing the main variables of interest [72].

The processors used for the analysis are briefly introduced in the following. For a more detailed description refer to the work of the main authors of the framework $[73,74]$.

\section{Data Unpacking}

The basic calibration processor chains start with reading in the EUDAQ raw data with the EUDAQInputProcessor into a format that can be read by the following processors. These are the Unpacker processors which for specific detector types interpret the data and store them in raw hit collections in the LCIO file. After this step a charge calibration from the detector units [ToT] into a more physics motivated unit as [e] can be conducted with the newly implemented PixelChargeCalibrator processor. It takes as input a ROOT TF1 function and parameters for it for each pixel, and applies the function to every raw hit. The converted charge value is stored then instead of the detector response.

\section{Hot Pixel Masking}

Noisy or hot pixels that fire even in the absence of particles are masked in the first step of the calibration with the HotPixelKiller, because they provide no information. To detect theses pixels the relative occupancy of every pixel in every detector is calculated for a set of events. If the occupancy is larger than a predefined threshold, typically $1 \%$, the pixel is marked as noisy and stored in the hotpixelDB (Data Base). The following processor chains use this DB to mask the pixels.

\section{Clustering and Hit Making}

The raw hits in the collection are grouped into clusters to collect the charge of a transitioning particle of all hit pixels. The PixelClusterizer processor is used for this. Going through the raw hit collection, a new cluster is assigned if there are no other clusters in the neighbourhood, otherwise the raw hit is added to the nearest cluster and potentially now connected clusters are merged to one. The neighbourhood can be defined by common pixel edge of the raw hits or with a distance from the pixel centre. The cluster collection is stored to disk to be used in the further calibration steps.

For every cluster, a hit position $\left(u_{h i t}, v_{h i t}\right)$ in the local coordinates of the detector plane is constructed. There are two approaches implemented in TBSW. The simpler one is the centre-of-gravity algorithm in the CogHitmaker processor. It computes a weighted mean of the raw hits in the cluster with their collected charge, which is then assigned as the hit position.

The more sophisticated approach uses track information and cluster shapes, which is implemented in the GoeClusterCalibrator. For each cluster shape the resulting track intersections are transformed into the cluster shapes coordinate system. From the distribution the mean track position is used as estimator for the hit position in the different 
cluster shapes. Also the full covariance matrix for the hit positions can be calculated in contrast to the centre-of-gravity algorithm. The results of the cluster calibration are stored in the clusterDB, and are used by the GoeHitmaker to calculate the hit positions of the clusters.

This approach is an iterative process, because it relies on good tracks in an aligned telescope, which relies on the hit information. Therefore a first calibration of the telescope is performed with the centre-of-gravity hit positions as a starting point for the iterations of cluster calibrations and alignment of the telescope. It has been shown that this approach leads to an improved quality of the fitted tracks [74].

\section{Track Reconstruction}

The track model in TBSW is a 3D straight line with kinks at sensor planes and at air in the middle between sensor planes. The tracks are seeded by extrapolating a straight line from combination of hits from the first two planes in the beam. Because the beam divergence is typically low, in the order of 1-2 mrad at DESY test beams, track candidates with large slopes can be discarded, reducing the computation time needed.

For track finding and fitting forward and backward Kalman filters are used to predict the track state on a plane $k$ based on the previous or following planes in the beam, respectively, considering measurement uncertainties from the hit position estimation and multiple scattering at the sensors and in the air [75]. The track state on plane $k$ is updated with the measured hit information, if a hit with residual below a user defined cut-off exists. This is done iteratively for all planes in the beam and repeated for all seed tracks, yielding a list of track candidates.

The forward and backward Kalman filter are combined in the track fitting process to precisely predict the track state at one plane using all the others. As a quality criterion for the track the $\chi^{2}$ value can be calculated as a sum of the squared residuals of the predicted state and the hit position and their covariance of every plane. The $\chi^{2}$ distribution of the fitted tracks should have a mean value equal to the degrees of freedom $j$ of position measurements in the telescope, and a variance of $\sigma^{2}=2 j$.

The Kalman filter is implemented in the FastTracker processor. It is possible that multiple track candidates share the same hit on a sensor plane. To resolve this a cut on the $\chi^{2}$ values is applied. From the remaining candidates the one with the lowest $\chi^{2}$ value is kept as the reconstructed track and the rest is discarded.

\section{Alignment}

A precise knowledge of the position of every plane in the telescope relative to the others is important for the quality of the track finding. At the test beam normally the positions of the planes in beam direction $z$ are measured with millimetre precision. The other parameters $x, y$ in the plane and the rotation angles of the plane $\alpha, \beta$, and $\gamma$ are fixed by the setup, but are undetermined. Starting from the $z$ positions and the other values set to zero, the alignment is iteratively determined using tracks, and with the results the alignment $D B$ is updated. 


\section{Test Beam Measurements with Diamond Pixel Modules}

As a first step a pre-alignment is carried out with the Correlator processor. From the hits on a fixed plane, the hit positions on the other planes are extrapolated along the beam axis. If the telescope is aligned, the residuals of the estimated and measured hit positions are centred at $0 \mathrm{~mm}$ in the $x$ and $y$ direction. As a first estimation of the alignment constants between the planes, the mean of the residual distributions is stored in the DB.

The processor used to calculate the final alignment corrections is the KalmanAligner. For a set of tracks, a global $\chi^{2}$, consisting of the residuals of the track at every plane and the difference of the updated alignment to the initial one considering the uncertainty of the initial one, is minimised. The TrackFitter loads the alignmentDB, and a very loose $\chi^{2}$ criterion is applied on the tracks to find tracks in a misaligned telescope. To stabilize the fitting procedure, preventing failed minimisations, first the more sensitive position parameters $x, y$, and $\gamma$ are free to be optimised and the others fixed by setting their initial uncertainties to zero. This alignment step is iterated twice, updating each time the alignment $D B$ with improved values for $x, y$, and $\gamma$.

In a second step, a track sample with tighter $\chi^{2}$ cut is used, because by now the alignment should be good enough to find good track samples. With this, the less sensitive parameters $z$, and even $\alpha$ and $\beta$ are aligned and the alignmentDB updated with their values. This is iterated three times.

If the cluster shape calibration algorithm is used to determine the hit positions, the alignment and the calibration optimisation have to be iteratively interleaved, because both depend on good track samples and therefore on each other.

\section{Geometry Description}

The geometric setup of the telescope is stored in an XML file in TBSW. It contains the position information of the detectors and information about the detectors such as material parameters and pixel matrix design. During the work of this thesis, the Marlin interface of reading and storing the geometry information for the other processors is upgraded $^{5}$ to match the requirements of describing the pixel layout of the diamond sensors.

The layout of the 3D diamond sensor consists of rectangular and staggered hexagonal pixels, as described earlier in Section 6.1.2. In TBSW only rectangular pixels are used to form a pixel matrix according to the pitch of the pixels. Different approaches were followed approximating the layout with rectangular pixels and using the cluster shape calibration algorithm to define the hit position in the pixels. As additional complication, the pixels in the layout have to be matched to the connected read-out pixel, whose column and row numbers are used in the data to encode the position of the hit. Because of the routing of the read-out pixel to the sensor pixel, the definition of "neighbouring" by adjacent column and row values is no longer valid, which is a problem for the clustering

\footnotetext{
${ }^{5}$ Structural changes in TBSW by the main author Benjamin Schwenker, and the implementation of the polygonal pixel class and a first script to create the XML files consistently by the author of this thesis.
} 
algorithm. In the end the geometry description was upgraded to a very flexible design accounting for all these problems.

To implement arbitrary pixel matrices consisting of polygonal shaped pixels, the ROOT class TH2Poly is used. It is designed to allow for polygonal bins in a two dimensional histogram. The pixel edge coordinates are filled into a TGraph object with the centre at zero as the design charge collection spot, which is then placed as the pixel in the layout. The name of the TGraph pixel can be accessed even afterwards, and is used to store the prototype pixel, see later, and the column and row index of the associated read-out pixel, implementing the matching between sensor and read-out pixel. The TH2Poly class has a check function implemented to return which bin (pixel) contains the given coordinates. This allows for matching track incident positions to pixels comparing the coordinates of both.

The TH2Poly object is the basis for the PolyDet class, which describes the layout of the pixel matrix and the neighbourhood definition needed for the clustering algorithms. It also contains functions to get sensor pixel information from read-out pixel values as input and vice versa, and general information about the layout. To not store too many redundant pieces of information, the pixels are grouped into so called prototype pixels, which have the same shape, and relative positioning in the matrix compared to the other pixels. For each prototype pixel, the edge points of the pixel, the prototype pixel number, and the maximum distance in $u$ and $v$ defining the neighbourhood area, are stored. The pixels themselves then need only to provide their prototypes, the positions in the layout, and the associated columns and rows of the read-out chip to be stored in the layout as TGraph bin. With this approach, non pixelated areas are possible and return the prototype pixel number -1 if queried.

The Geometry processor is implemented to contain the PolyDet class and the implementation for the rectangular pixels, the SquareDet class. For most applications the overhead of the PolyDet class is not needed, and the SquareDet class is the default choice. This new processor is added to every processing chain, providing the telescope layout consistently in one location. The information needed for the processor is still stored in XML files, and the implementation is backwards compatible. A mixture of detectors defined with the two classes in one telescope description is possible. Because a lot of parameters have to be stored in the XML files for the PolyDet class, a python script is implemented, providing the user an interface, where the parameters for the prototype pixels and the pixels for each plane, and the general structure of the telescope have to be specified, to create an XML file. The script is not limited to the PolyDet class, but can be used equally for telescopes with both SquareDet and PolyDet detectors.

With this implementation, the layouts of the two diamond samples can be described, the combination of rectangular and hexagonal pixels in the 3D diamond module, as well as the polygonal shaped traces in the planar diamond module. In Figure 6.3(b) and Figure 6.4(b), the layouts were already presented. 


\section{Test Beam Measurements with Diamond Pixel Modules}

\subsubsection{Data Calibration and Reconstruction}

For the calibration of the telescope, at least $300 \mathrm{k}$ events are used. In the following, the used processors in the calibration chains and the reconstruction are listed. As described above, the Geometry processor is needed in every chain and therefore not extra mentioned. Almost the same steps are used for the analysis of the planar and 3D diamond modules. In case of deviations, these are mentioned, otherwise the list applies to both.

\section{Telescope Calibration}

1. Hot pixel masking

The EUDAQInputProcessor and the NI- and USBPixUnpacker are used to read the data of the MIMOSA26 telescope detectors and the FE-I4 reference and diamond detectors. Then the HotPixelKiller processor writes the hot pixels with a relative occupancy above $0.1 \%$ to the hotpixelDB.

2. Clustering

The data is read in again as for the masking step. For the planar diamond data, the raw hits are first calibrated with the PixelChargeCalibrator with a calibration obtained with an STControl routine, and then also clustered with the PixelClusterizer processor as for the $3 \mathrm{D}$ diamond data. The clusters are saved in an LCIO file.

3. Correlator

The cluster collections saved in the clustering step are used with the CogHitmaker processor to create hit collections for the Correlator processor. The first plane in the beam is set as reference plane. The first alignment values for $x$ and $y$ are written in the alignment $D B$.

4. Pre-alignment

Again the cluster collections are loaded and hit collections computed. Then the FastTracker processor is used with loose cuts to create a first track collection. The cuts are $\chi^{2}=10^{7}$ and maximum residuals of $500 \mu \mathrm{m}$ in both plane directions. New alignment values for $x, y$, and $\gamma$ are calculated with the KalmanAligner and stored in the DB.

5. Alignment

The same setup as for the pre-alignment is used, only the cuts for the FastTracker are tightened, because a basic alignment for good tracks is present, and the updated alignmentDB is loaded. The cuts are $\chi^{2}=100$ and maximum residuals of $400 \mu \mathrm{m}$ in both plane directions. Then the alignment constants for all parameters are calculated, but the $\alpha$ and $\beta$ values only for the reference and the diamond module, because the analysis is not that reliant on these angles. This processor chain is carried out three times. 
6. Cluster calibration for the 3D diamond module

The cluster calibration process is used for the 3D diamond module and not the planar diamond module to keep the assigned hit positions at the design pixel centres, at least for single hit clusters, to better understand the implications of the more difficult trace pixel layout. It is an iterative process of alignment and calibration.

a) Pre-cluster calibration

The same chain as for the alignment is used except that the diamond module is excluded, and the KalmanAligner is replaced with the GoeClusterCalibrator. The clusterDB is filled with the cluster shapes of the MIMOSA26 and reference detectors.

b) Telescope alignment

The clusterDB is used with the GoeHitmaker to create hit collections for the MIMOSA26 and reference sensors. Then the hits are used in a track finder with the tight cuts, excluding still the diamond detector. The tracks are used in the KalmanAligner to update the alignment. This is repeated three times.

c) Cluster calibration

From the clusters now for all detectors the GoeHitmaker is used to create the hit collections. With this input, the track finder with tight cuts, including the diamond module, provides the track collection for the cluster calibration. This is done six times.

d) Alignment

The alignment is carried out as before, three times, to create the final alignment.

\section{Reconstruction}

The reconstruction is done for every event and results in the filling of ROOT TTrees with the hit and track information of the reference and the diamond detectors. It is only one chain of processors, which uses the DBs created during the calibration.

The data are read in as for the calibration: EUDAQInputProcessor, Unpackers, PixelChargeCalibrator for the planar diamond, and the PixelClusterizer processor. Then the CogHitmaker is used for the planar diamond, and the GoeHitmaker for the 3D diamond. The FastTracker with tight cuts creates the track collection. The diamond detector is excluded in the track finding in order not to bias the later measurements. The track and the hit collections are matched on the diamond and on the reference detector with the PixelDUTAnalyser by applying a residual cut of $400 \mu \mathrm{m}$ for the diamond and $200 \mu \mathrm{m}$ for the reference detector. This yields the later used matched tracks and hits. The results are stored in TTrees in ROOT files for the analysis. 


\section{Test Beam Measurements with Diamond Pixel Modules}

\subsection{Results}

In the first section the telescope and the reference sample are qualified to lay the basis of the following analysis of the diamond detectors.

\subsubsection{Reference Sample and Telescope Resolution}

The alignment quality of the telescope is assessed with the residual distributions of the reference detector. In Figure 6.8, the residual distribution in $u$ direction is displayed for single pixel hits. The edges of each distribution are fitted with complementary error functions $\operatorname{erfc}(x)$ to extract the width of the distribution and the resolution $\sigma$ :

$$
\begin{gathered}
f\left(\text { Scale }_{\text {rise }}, x_{\text {rise }}, \sigma_{\text {rise }} ; x\right)=\text { Scale }_{\text {rise }} \cdot \operatorname{erfc}\left(\frac{x_{\text {rise }}-x}{\sqrt{2} \sigma_{\text {rise }}}\right), \\
f\left(\text { Scale }_{\text {fall }}, x_{\text {fall }}, \sigma_{\text {fall }} ; x\right)=\text { Scale }_{\text {fall }} \cdot \operatorname{erfc}\left(\frac{x_{\text {fall }}-x}{\sqrt{2} \sigma_{\text {fall }}}\right) .
\end{gathered}
$$

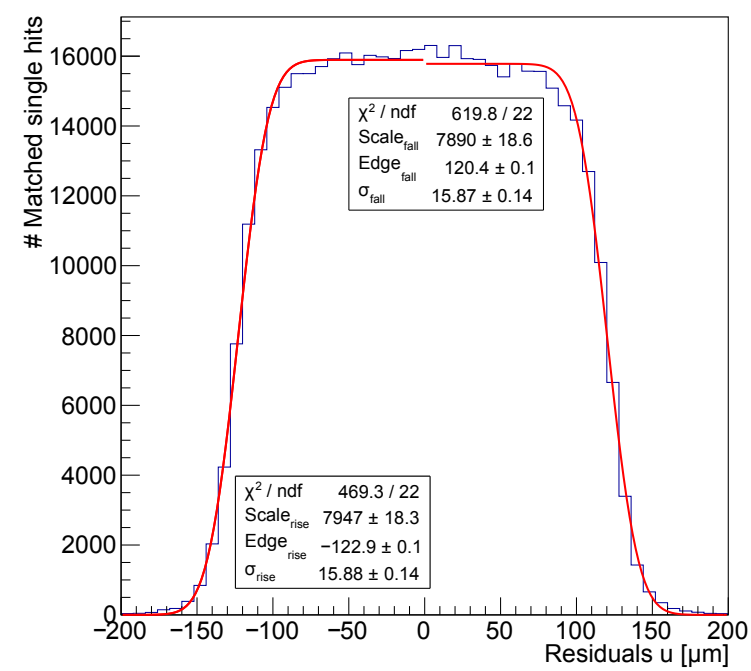

Figure 6.8.: Residual distributions for the reference detector $u$ direction measured from the data set for the planar diamond detector at $400 \mathrm{~V}$ bias voltage. Fit according to (6.1).

In Table 6.5, the results of the fit as the width of the distributions, $x_{\text {fall }}-x_{\text {rise }}$, and the $\sigma_{\mathrm{u}}$ are listed averaged for same beam conditions. The widths reflect the pixel sizes in both direction, the difference to the nominal values of $250 \mu \mathrm{m}$ in $u$ direction and $50 \mu \mathrm{m}$ in $v$ direction can be explained with charge sharing at the pixel edges. No significant position deviations are observed, which shows a consistent calibration. The track resolution is estimated with the $\sigma_{\mathrm{u}}$ value only, because the $v$ direction is more influenced by multi hit clusters due to the smaller pixel size in that direction, and the resolution is assumed to be isotropic. As expected, the resolution is better for a higher beam energy comparing 


\begin{tabular}{|c|c|c|c|c|}
\hline Test beam & Beam energy $[\mathrm{GeV}]$ & $u$ width $[\mu \mathrm{m}]$ & $v$ width $[\mu \mathrm{m}]$ & $\sigma_{\mathrm{u}}[\mu \mathrm{m}]$ \\
\hline Planar diamond & 4.6 & $243(1)$ & $45(1)$ & $16.0(4)$ \\
\hline Planar diamond & 4.0 & $246.3(1)$ & $46.3(6)$ & $18.2(3)$ \\
\hline 3D diamond & 4.0 & $249(4)$ & $47(1)$ & $15.3(6)$ \\
\hline
\end{tabular}

Table 6.5.: Results of the residual fits for the reference detector average for same beam conditions.

the data sets for the planar diamond. The resolution is better for the 3D diamond data set despite the lower beam energy, same reference sample and similar telescope. The usage of the cluster calibration algorithm for the $3 \mathrm{D}$ diamond data set could be the cause, improving the hit position estimation and the track finding.

\begin{tabular}{|c|c|c|c|}
\hline Test beam & Bias diamond $[\mathrm{V}]$ & Beam Energy $[\mathrm{GeV}]$ & $\epsilon_{\mathrm{ref}}[\%]$ \\
\hline Planar diamond & 50 & 4.6 & $98.2(2)$ \\
\hline Planar diamond & 100 & 4.6 & $98.09(6)$ \\
\hline Planar diamond & 150 & 4.0 & $99.42(3)$ \\
\hline Planar diamond & 200 & 4.6 & $98.13(4)$ \\
\hline Planar diamond & 250 & 4.6 & $98.13(3)$ \\
\hline Planar diamond & 300 & 4.6 & $98.18(4)$ \\
\hline Planar diamond & 350 & 4.6 & $98.27(3)$ \\
\hline Planar diamond & 400 & 4.6 & $98.16(2)$ \\
\hline Planar diamond & 500 & 4.6 & $98.11(3)$ \\
\hline Planar diamond & 600 & 4.0 & $99.47(3)$ \\
\hline Planar diamond & 600 & 4.6 & $99.52(2)$ \\
\hline 3D diamond & 50 & 4.0 & $99.51(5)$ \\
\hline 3D diamond & 60 & 4.0 & $99.39(6)$ \\
\hline 3D diamond & 70 & 4.0 & $99.32(8)$ \\
\hline
\end{tabular}

Table 6.6.: Efficiency of the reference detector $\epsilon_{\text {ref }}$ for the different test beam measurement data sets.

The global efficiency $\epsilon$ for a detector is defined as the ratio of observed tracks to expected tracks in a fiducial volume:

$$
\epsilon=\frac{\text { \#observed tracks } \mid \text { fiducial region }}{\text { \# expected tracks } \mid \text { fiducial region }} .
$$

For the expected tracks, an associated hit on the reference detector is required for the timing of the event. The efficiency of the reference sample uses the information of the diamond detector as reference. The statistical uncertainties are calculated within the ROOT TEfficiency class with Bayesian statistics using the binomial distribution and a uniform prior. In Table 6.6, the results based on all tracks seen by the diamond detector for the different settings of the measurements can be found. The efficiency is above 


\section{Test Beam Measurements with Diamond Pixel Modules}

$98 \%$ for all data sets recorded. Only a small area of the reference detector behind the diamond can be used for the estimation, because the diamond has a small size. The position of the reference module was arranged to have little problematic pixels in that area. Considering the high efficiency, no bias towards the diamond detectors is expected from the reference module.

\subsubsection{Planar Diamond}

The planar diamond detector is a test bench for the 3D diamond detector. For the first time the bump bonding of the diamond to the FE-I4 read-out chip was successfully performed at the IFAE institute in Barcelona, qualifying it for building further detectors with $3 \mathrm{D}$ electrode geometry. Furthermore, the charge collection properties of the metallisation without $3 \mathrm{D}$ electrodes is investigated as reference for such electrode configurations.

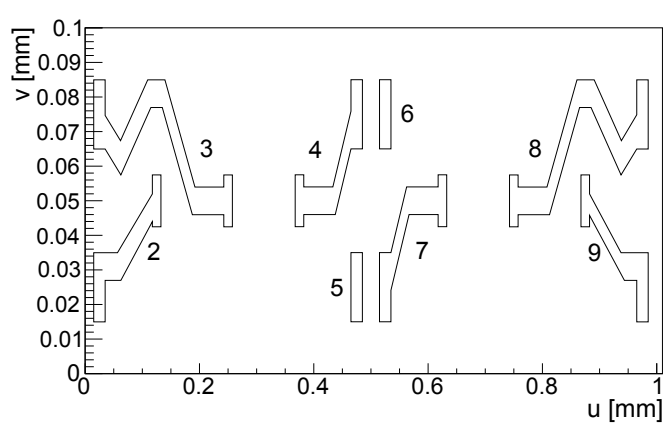

(a)

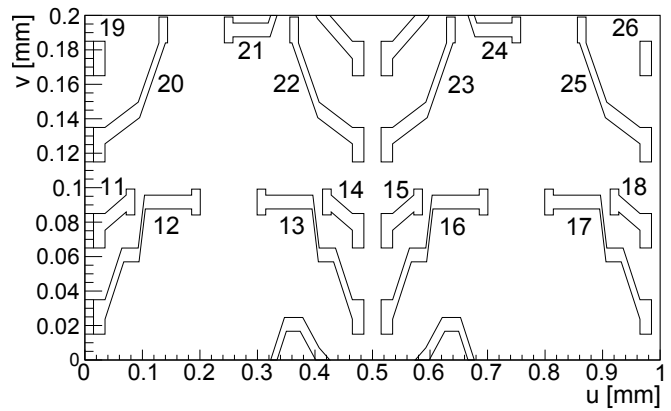

(b)

Figure 6.9.: Super-cells for the planar diamond pixel matrix for the rectangular and the hexagonal region of the metallisation in (a) and (b), respectively. The lines show the traces and the numbers indicate the prototype pixels.

The pixel layout of the planar diamond module is built in TBSW with 28 prototype pixels, each representing a unique trace in a unique surrounding. The nominal centre of the pixels is assigned on the pixel in $v$ direction at the centre in $u$ direction. In Figure 6.9, the prototype pixels are grouped to the smallest repeating combination in the layout, which is called a super-cell. With periodic repetition the full layout can be described with these super-cells. There are two different super-cells reflecting the rectangular and hexagonal pixels in the 3D diamond layout, respectively. The areas of the metallisation built from the super-cells are called also rectangular and hexagonal regions for simplicity. In the plots of the super-cells the prototype pixels are indicated with their number as reference for the later analysis. The missing prototype pixels in the display, $0,1,10$, and 27 , are the two prototype pixels for the non metallised FE-I4 bump bonds connected to the diamond, and two filler pixels at the edge of the metallisation.

The number of reconstructed tracks in the data sets are displayed in Table 6.7. For the analysis the selection of single tracks in the sensitive area of the DUT with a hit on 


\begin{tabular}{|c|c|c|c|c|c|}
\hline $\begin{array}{c}\text { Bias voltage } \\
{[\mathrm{V}]}\end{array}$ & $\begin{array}{c}\text { \# Events } \\
{[\text { Mio] }}\end{array}$ & $\begin{array}{c}\text { \# Tracks } \\
\text { on DUT [k] }\end{array}$ & $\begin{array}{c}\text { \# Good } \\
\text { tracks [k] }\end{array}$ & $\begin{array}{c}\text { \# Good tracks } \\
\text { (rect) }[\mathrm{k}]\end{array}$ & $\begin{array}{c}\text { \# Good tracks } \\
\text { (hex) }[\mathrm{k}]\end{array}$ \\
\hline \hline 50 & 1.6 & $203.8(5)$ & $42.5(2)$ & $9.9(1)$ & $10.5(1)$ \\
\hline 100 & 3.5 & $690(1)$ & $144.7(4)$ & $33.7(2)$ & $35.6(2)$ \\
\hline 150 & 2.2 & $622(1)$ & $80.0(3)$ & $18.9(1)$ & $20.6(1)$ \\
\hline 200 & 4.9 & $941(1)$ & $204.2(5)$ & $47.9(2)$ & $50.3(2)$ \\
\hline 250 & 6.2 & $1181(1.1)$ & $252.5(5)$ & $59.0(2)$ & $62.3(2)$ \\
\hline 300 & 5.5 & $1060(1)$ & $221.7(5)$ & $51.5(2)$ & $54.5(2)$ \\
\hline 350 & 7.6 & $1401(1.2)$ & $313.5(6)$ & $72.8(3)$ & $77(3)$ \\
\hline 400 & 17.5 & $3366(2)$ & $712.4(8)$ & $165.4(4)$ & $176.3(4)$ \\
\hline 500 & 6.1 & $1194(1.1)$ & $251.1(5)$ & $58.8(2)$ & $62.2(2)$ \\
\hline $600(4.0 \mathrm{GeV})$ & 2.6 & $745(1)$ & $96.2(3)$ & $22.8(2)$ & $24.8(2)$ \\
\hline $600(4.6 \mathrm{GeV})$ & 1.5 & $327.1(6)$ & $70.8(3)$ & $17.0(1)$ & $18.0(1)$ \\
\hline
\end{tabular}

Table 6.7.: Track statistic for the data sets with the planar diamond module. Good tracks are single tracks on DUT sensitive area with a hit on the reference module, for the last two columns split in the rectangular and hexagonal pixel areas, respectively.

the reference module is used to simplify the matching of tracks and hits, and to assure the correct timing of the tracks. The low number of tracks compared to the number of events is explained with the larger trigger area compared to the small area of the diamond, and taking into account the number of events with multiple tracks. The track counts for the different pixel areas reflect the $6 \%$ larger hexagonal area compared to the rectangular area.

Representative for the analysis steps, the data of the measurements with an applied bias voltage of $500 \mathrm{~V}$ is displayed in the following. This data set has a larger amount of events compared to higher bias voltage settings and in this range of the bias voltage differences in the behaviour of the diamond should be small. Distinct differences in other data sets are presented to characterise the diamond module completely.

\section{Matched Track Positions}

The matched track positions for the planar diamond detector are evaluated in the $u v$ plane individually for each prototype pixel, because the pixel shapes can not be represented as residuals in $u$ or $v$ direction alone and the pixels themselves are not comparable. Relative to the matched hit position the track positions are mapped for each prototype pixel. A full set of maps of all prototype pixels for the bias voltage of $500 \mathrm{~V}$ can be found in Appendix A.2.2. Here a selection of the prototype pixels 8 and 9 for the rectangular region and prototype pixels 23 and 24 for the hexagonal region are displayed in Figure 6.10. As guidance, the shape of the pixel trace is overlayed in the plots as well as nearby traces. The core of the track intersection distribution follows the trace with the maximum values at the centre of the trace. This shows that the particles detected 


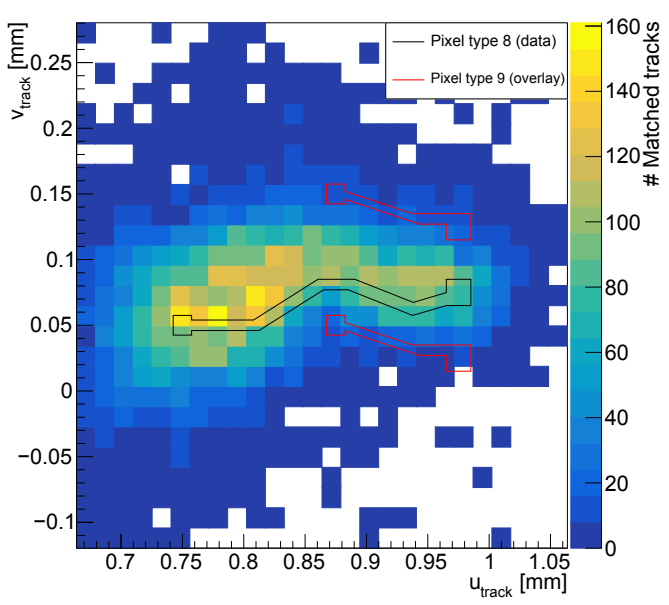

(a)

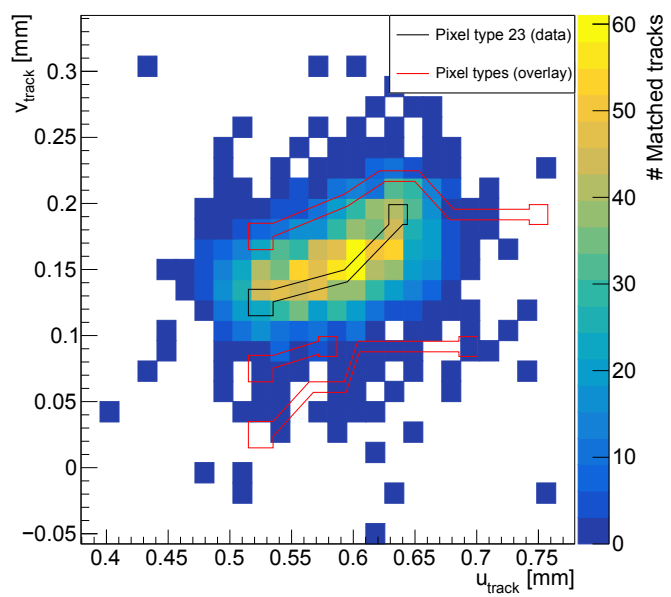

(c)

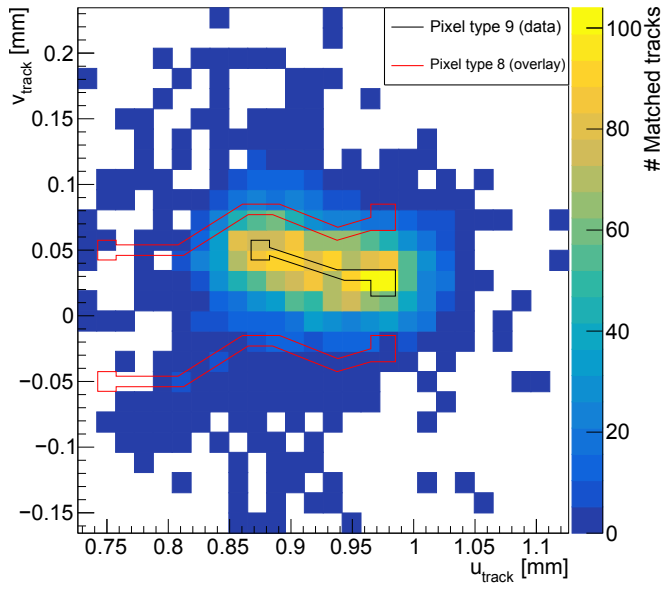

(b)

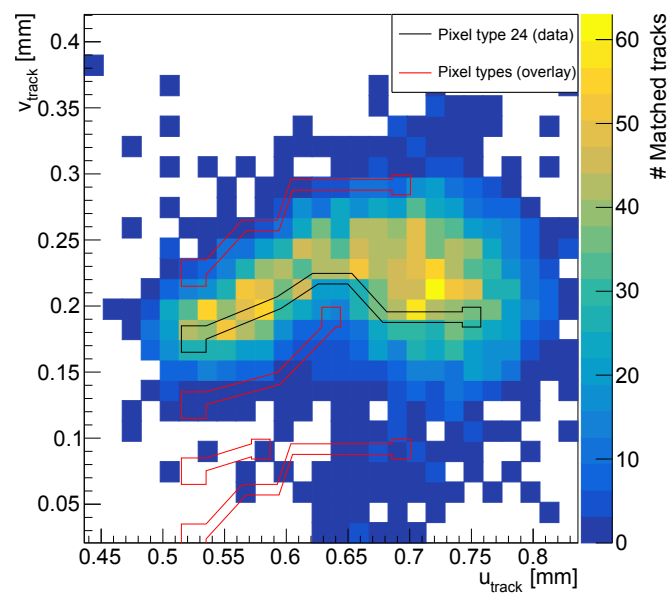

(d)

Figure 6.10.: Map of matched track positions with hit in read-out connected to prototype pixel 8 and 9 from the rectangular region in (a) and (b), and for prototype pixel 23 and 24 in (c) and (d), respectively. Track positions are relative to prototype pixel position in super-cell. Overlay with read-out trace of the hits (black) and nearby traces (red). Measured with planar diamond at $500 \mathrm{~V}$ bias voltage.

create their charge in the area around the trace and are then collected with the trace and routed to the read-out pixel.

The collection region around the trace is influenced by the nearby traces. For the prototype pixel 24, this can be seen in Figure 6.10(d). The maximum of the track position distribution is shifted away from the trace, most notable where the trace is bent to leave space for the prototype pixel 23. In this area the most tracks are recorded with prototype pixel 23, which itself is limited on the other side by the prototype pixel 15 
and 16 .

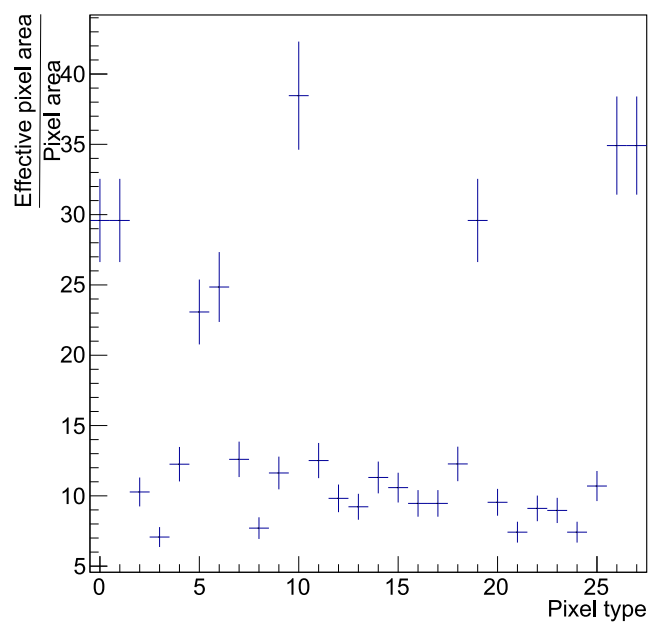

Figure 6.11.: Ratio of the effective pixel area to the pixel area defined by the trace for the prototype pixels, biased with $500 \mathrm{~V}$.

To estimate the charge collection region around the traces, the area of the bins with more then $0.5 \%$ of the number of entries in the plots is summed up as an effective pixel area and compared to the area of the trace. A relative uncertainty of $10 \%$ is assigned to the effective area to account for fluctuations around the threshold. In Figure 6.11, the ratio of the effective area and the trace area is displayed for the different prototype pixels. A split of the prototype pixels in two groups is observed with ratios around 9 and and 28 , respectively. The prototypes with higher ratio are all the pixels with only a metal pad at the bump bond or, in case of prototype 0 and 1 , only the bump bond. As a combination of small pixel size and larger distance to other traces, the effective pixel area can extend further for these pixels especially for prototype pixel 0 and 1 with no other traces around. The other set of prototype pixels has a similar increase of the pixel area around the traces for each prototype. The limited space between the traces levels out the areas of influence. This level off is confirmed by the observation that the ratios stay the same within uncertainty for all bias voltages applied. The only exception are the measurements for the bias voltage of $50 \mathrm{~V}$, for which the ratios of the first group of pixels with small traces are closer to the other ones, whose ratios are more spread out in general. For $50 \mathrm{~V}$ bias voltage, the areas of charge collection around the traces seem not to be maximised as they are not influencing each other much.

The charge collection areas for the different prototype pixels in the super-cells can be estimated by plotting the relative matched track positions centred at the prototype pixel position in the super-cell area and inserting the associated hit prototype pixel number times the hit count. Then the bin values are averaged by the number of entries in the bin. This is displayed in Figure 6.12 for the rectangular and hexagonal super-cell. In 


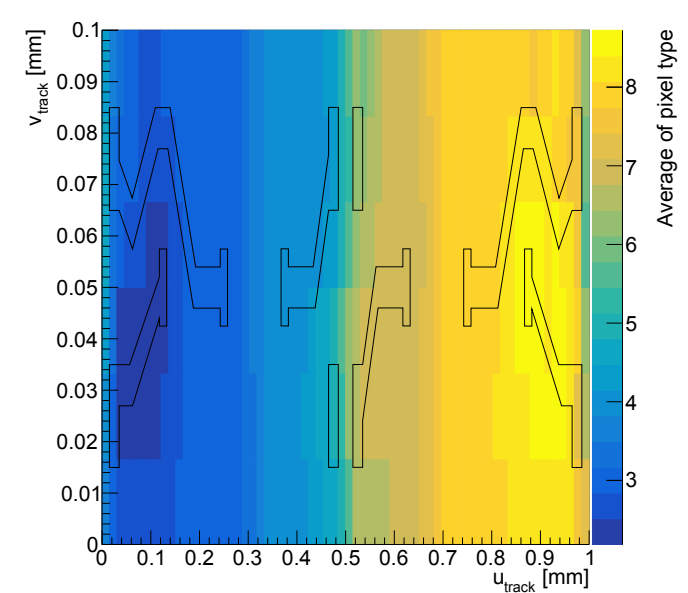

(a)

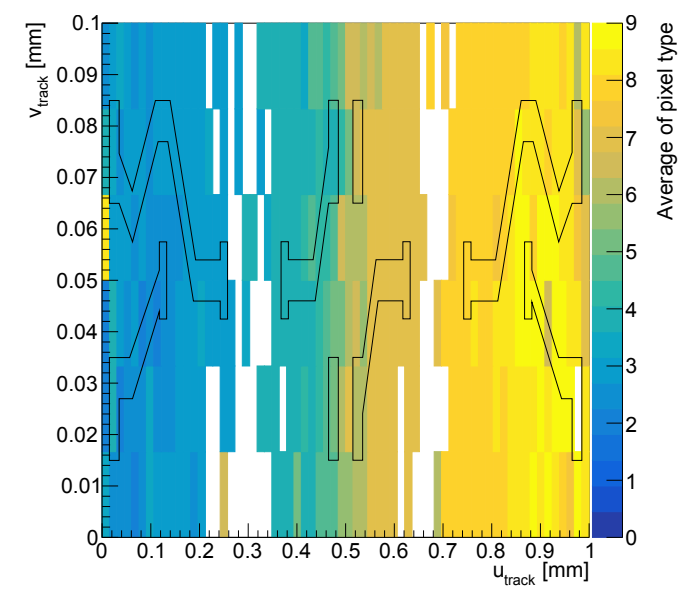

(c)

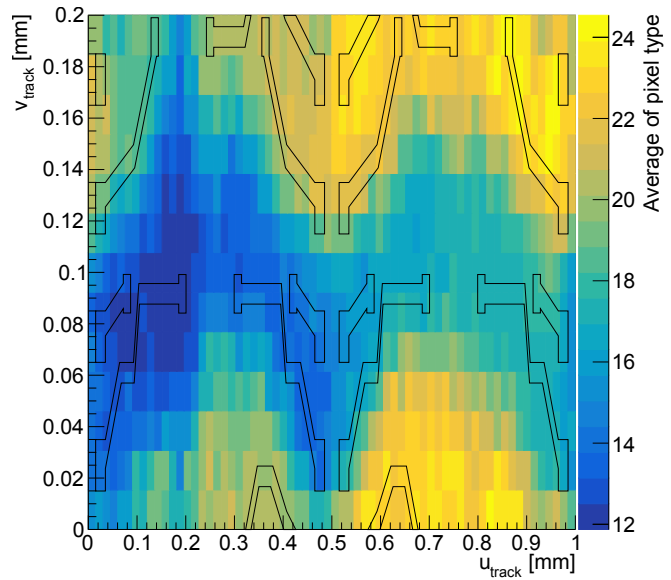

(b)

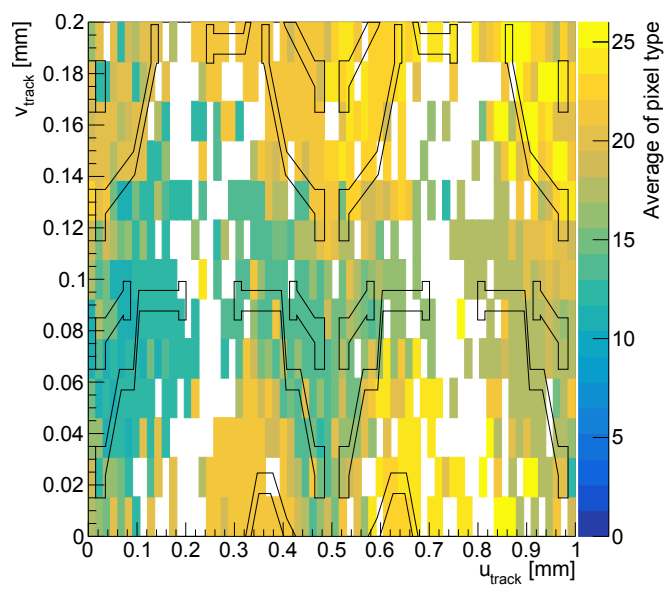

(d)

Figure 6.12.: Average prototype pixel number of the hit pixel at the position of the matched track folded into the rectangular (c) and hexagonal (d) super-cell. At $500 \mathrm{~V}$ bias voltage (first row) and at $50 \mathrm{~V}$ (second row).

the areas around the traces, the charge is collected by the read-out pixel connected to the trace. The boundaries of these areas are not sharply defined, and in the transition multiple pixels can collect the charges. A clear difference in the behaviour can only be observed for the measurements with $50 \mathrm{~V}$ bias voltage. There in the regions between the traces no particles are detected, which confirms the result from the effective pixel sizes that the maximum charge collection areas are not reached. With such a low bias voltage the charges created between the traces seem not to induce a large enough signal on them, because they are trapped in the low field regions.

This all shows that the traces act as pixels though they are not designed as such. Most of the matched tracks are recorded around the traces and with the read-out pixel 
connected to the trace.

\section{Charge Collection}

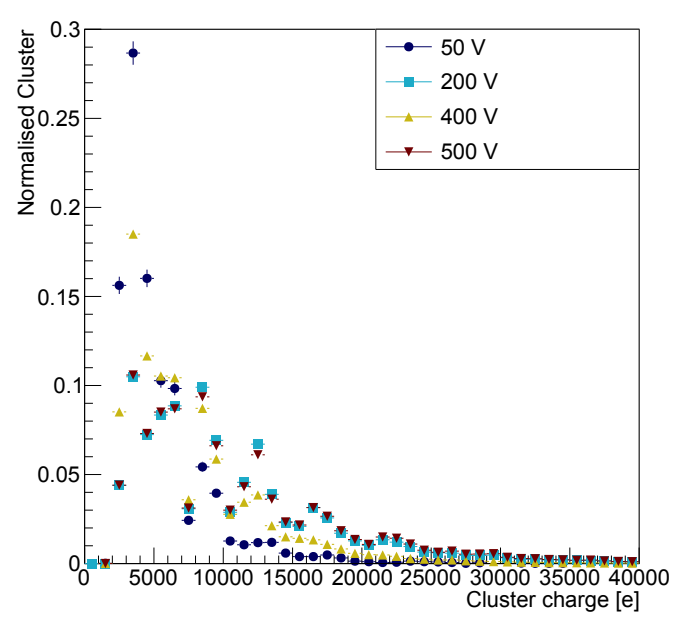

(a)

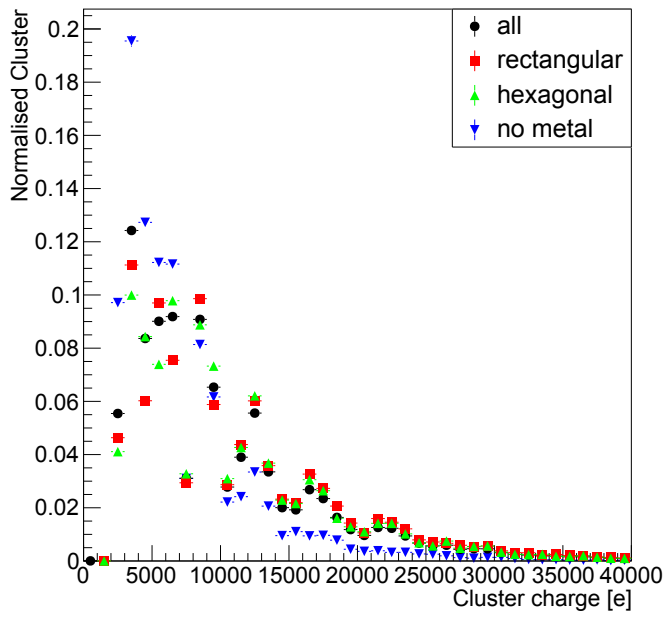

(b)

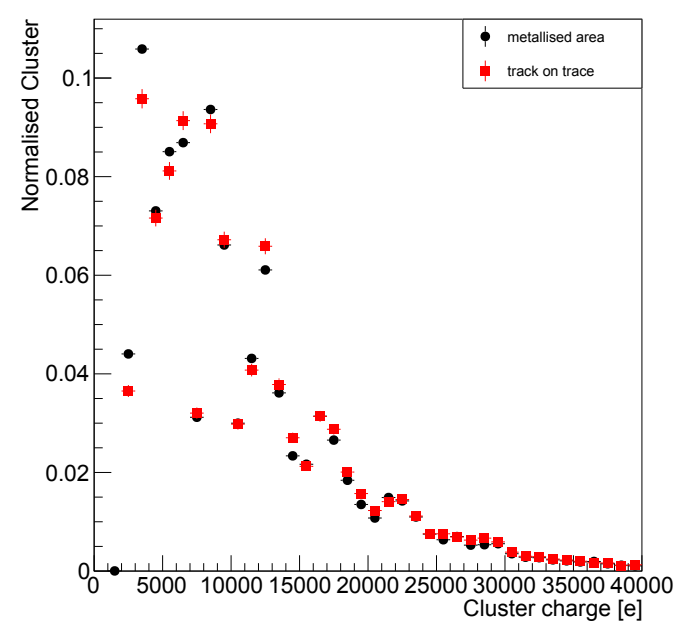

(c)

Figure 6.13.: (a): cluster charge of hits in the planar diamond detector. Cluster charge distributions for bias voltage of $500 \mathrm{~V}$ in (b) for different regions, and in (c) cluster charge comparison for metallised area to only trace area.

The FE-I4 ToT response is calibrated with a calibration scan from STControl, yielding parameters for a second order polynomial to transfer ToT to electrons. The cluster charge of the hits in the metallised area is compared in Figure 6.13(a) for the bias voltage $50 \mathrm{~V}$, $200 \mathrm{~V}, 400 \mathrm{~V}$ and $500 \mathrm{~V}$. These distributions are exemplary for the behaviour of the data sets, find the other data sets at different bias voltages in Appendix A.2.2. The peak 


\section{Test Beam Measurements with Diamond Pixel Modules}

of the distributions is in the second bin at 3 ke to 4 ke and the tail extends to about $30 \mathrm{ke}$. It is attempted to fit a landau-gauss convolution to the distribution to quantify differences for the different data sets, but the fit quality is too poor to rely on. For bias voltages of $200 \mathrm{~V}, 250 \mathrm{~V}, 500 \mathrm{~V}$, and $600 \mathrm{~V}$ more clusters with higher charge amounts are observed. The mean cluster charge is in the range of 9-10 ke for these voltages. This yields a CCD of $250-280 \mu \mathrm{m}$ with the expected charge of $36 \mathrm{e} / \mu \mathrm{m}$ for a m.i.p. in diamond, which matches the previous measured value of $260 \mu \mathrm{m}$. But many clusters with lower charge and the standard deviation of about 7 ke show that the charge collection is not stable. More clusters with less charge are observed for the other bias voltage settings, which is expected for the lowest bias voltages of $50 \mathrm{~V}, 100 \mathrm{~V}$ and $150 \mathrm{~V}$. Trapping effects are significant for $50 \mathrm{~V}$ even in comparison to $100 \mathrm{~V}$. But unexpected is the loss of charges for the middle bias voltages $300 \mathrm{~V}, 350 \mathrm{~V}$, and $400 \mathrm{~V}$. The potentially collectable charge should increase with bias voltage as the CCD increases with higher electrical fields. The chronological order of the measurements does not lead to such a behaviour, which could have explained it with deterioration or cumulative effects like polarisation in the diamond. Up till now no explanation for this observation has been found.

In Figure 6.13(b), the cluster charge distributions are compared for the different regions of the diamond. As expected, smaller signals are recorded in the region with only bump bonds connected to the diamond with the core of the distribution at 4-5 ke. Signals above 10 ke are observed with a similar rate in the rectangular and the hexagonal region. Below this charge, there are differences between the two regions but changing bin by bin which observes more clusters. An interplay of the different traces resulting in localised electrical field differences and trapping could be a reason but is not investigated further for this range of charges.

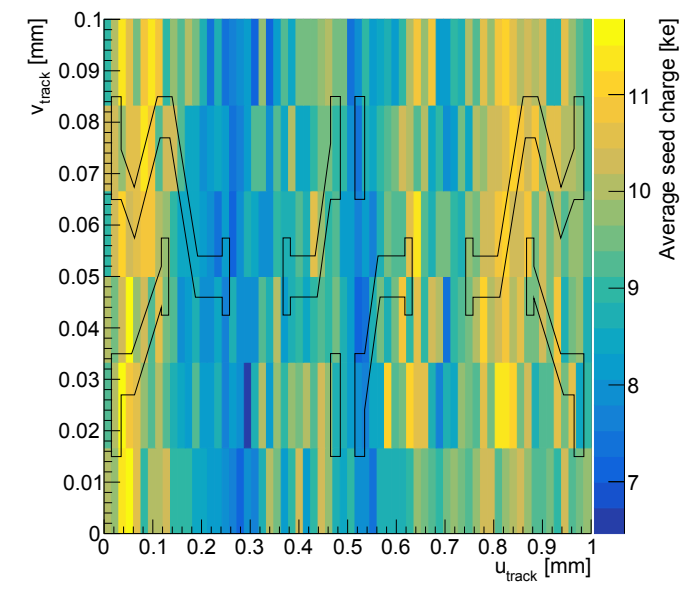

(a)

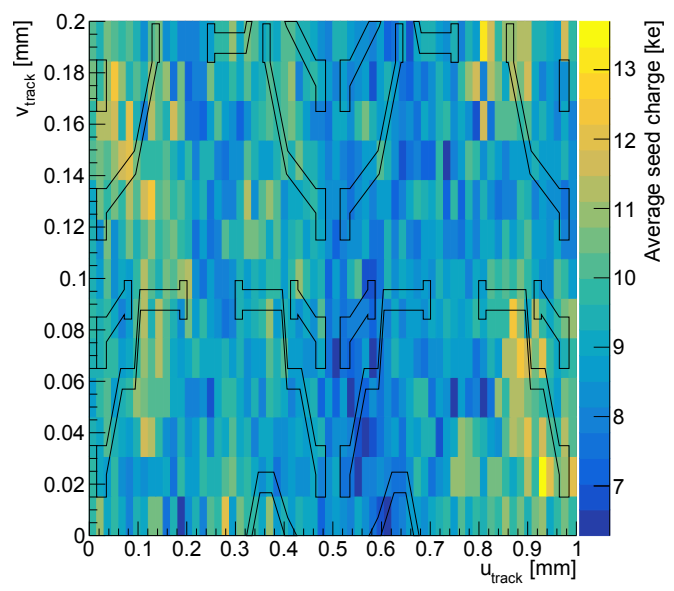

(b)

Figure 6.14.: Averaged hit seed charge at track position in rectangular super-cell (a), and in hexagonal super-cell (d). Bias voltage $500 \mathrm{~V}$.

Overall no improved charge collection is observed for tracks intersecting a trace, as 
can be seen in Figure 6.13(c). With the same method as for the map of the average prototype pixel in the super-cells, the average charge of the matched hit pixel is displayed in Figure 6.14(a) and Figure 6.14(b) for the rectangular and hexagonal super-cell, respectively. The range of the localised average charge considering all bias voltages is 4-16 ke, with the mean of the corresponding cluster charge distribution approximately in the middle of the range. Most of the spots with higher average charge are localised at the traces, showing that there is very local improvement of charge collection that averages out in the total cluster charge distribution for tracks on traces. This is consistent for the rectangular super-cell, although around the prototype pixels in the middle less charge is collected than at the other pixels. Also, in the hexagonal region at the central prototype pixels, 13-16, 23 and 24, the average collected charge is lower. The difference to the other traces are the prototype pixels 23 and 24, long traces to reach the 3D electrode furthest away from the bump bonds in the 3D diamond, curving around the other traces. The charges seem to be more split between the nearby traces in contrast to the other read-out columns where only a small metal pad leaves more distance to the other traces.

In total, charges are collected as expected for high bias voltages and trapping is observed for low bias voltages. At the traces on average a higher signal can be observed very localised depending on the influence of the surrounding traces.

\section{Efficiency}

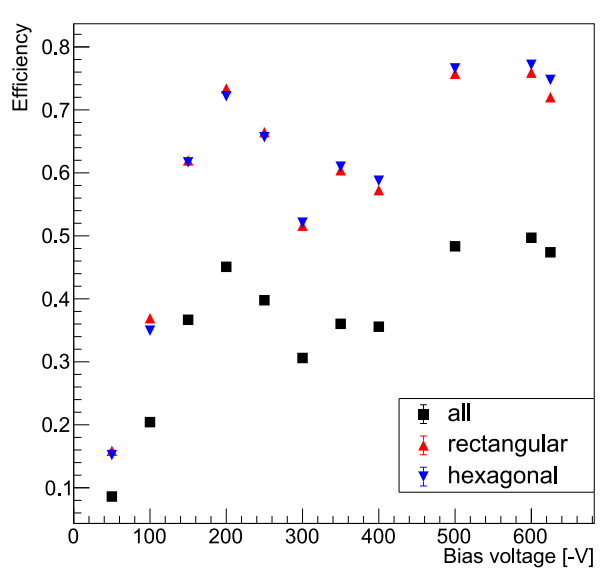

(a)

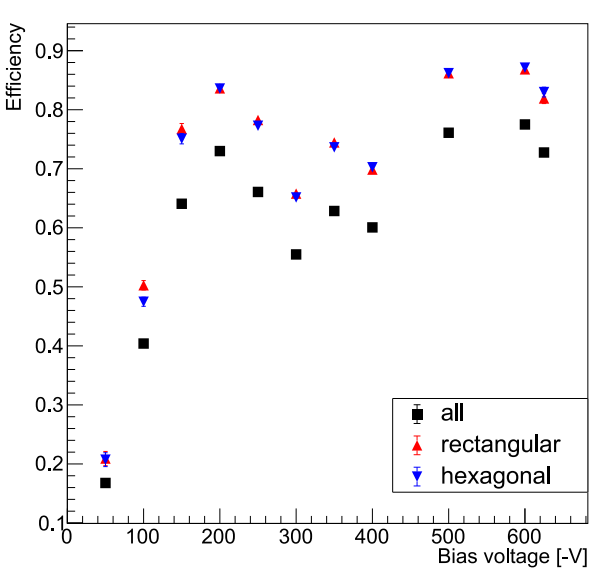

(b)

Figure 6.15.: Development of the efficiency with bias voltage compared for the fiducial regions full diamond, rectangular region, and hexagonal region. In (a) for all expected tracks in the region, and in (b) for expected tracks that intersect traces described in the layout. The measurement at $600 \mathrm{~V}$ and a beam energy of $4.6 \mathrm{GeV}$ is shifted to $625 \mathrm{~V}$. Uncertainties are smaller than the marker size. 


\section{Test Beam Measurements with Diamond Pixel Modules}

The efficiency definition from Equation (6.2) is used to estimate the efficiency for the different bias voltage settings split into the regions: full diamond, the rectangular region, and the hexagonal region. This is displayed in Figure 6.15(a). As expected, the efficiency in the full diamond is systematically lower than for the metallised regions only. Only particles passing near a bump bond in the non metallised area are detected, which is a small part of the diamond. A small deviation of up to $2 \%$ between the rectangular region and the hexagonal region is visible with the higher efficiency in the hexagonal region at higher bias voltages. For $50 \mathrm{~V}$ the electric field is clearly not enough to efficiently detect particles. Also for $100 \mathrm{~V}$ the efficiency stays below $40 \%$. The efficiency plateau starts at $200 \mathrm{~V}$ with only slight increase to the highest efficiency at the bias voltage of $600 \mathrm{~V}$, at a beam energy of $4.0 \mathrm{GeV}$, with $(76.0 \pm 0.2) \%$. At $500 \mathrm{~V}$ the efficiency is not significantly lower, but for $250 \mathrm{~V}$ to $400 \mathrm{~V}$ the efficiency is not stable and degrades by up to $20 \%$. These are the same bias voltages for which a reduction in collected charge is observed. A reduced charge collection can cause a reduced efficiency, which shows a consistent behaviour of the diamond during the specific measurements but no explanation for the base cause.

In Figure 6.15(b), the same comparison is made for the different data sets, but with the requirement that the expected tracks intersect the diamond at a trace, so the minimum pixel area. The efficiency increases with this selection by up to $10 \%$ in the rectangular and hexagonal region for all bias voltage settings. This shows that the charge collection is inefficient mainly in between the traces, even though charge is collected there as discussed before. For the full diamond region, this effect is even more visible considering the non metallised area. The difference between the pixelated regions and the full diamond reduces to about $10 \%$ from $20 \%$ to $25 \%$. In a limited range, charge collection with the bare bump bonds is possible. The remaining difference can be explained with systematically worse connections in the higher rows, also seen in the radioactive source measurements in the laboratory, see Figure 6.6(b).

Localised efficiencies are displayed in Figure 6.16, for all expected tracks at the track intersection binned for convenience in FE-I4 pixel size bins, and for the expected tracks intersecting a trace displayed in the layout of the traces. In the Appendix A.2.2, the data of the last mentioned plot is shown binned in the column and row of the expected read-out pixel for better visualisation of the entries in the traces. Both maps have similar amount of bins with very low efficiency mainly in the non metallised area for lower column and higher row values, as there the bump bonds are not connected well. In the opposite direction, good connections to the diamond are observed looking at the efficiency for trace tracks. The number of bins with efficiency close to $100 \%$ is three times higher for restricting the tracks to the traces, showing that the trace areas are efficient charge collection spots.

There are some areas in the metallised region of the diamond that have a systematically low efficiency. In Figure 6.17, the same efficiency map as before is shown alongside a map of visible defects in the diamond. The defect map uses the bias voltage grid as division, because it is a good orientation to map the defects, and most of the time the effect of the defect covers a cell of the grid. With the number 2 defects like dark spots 


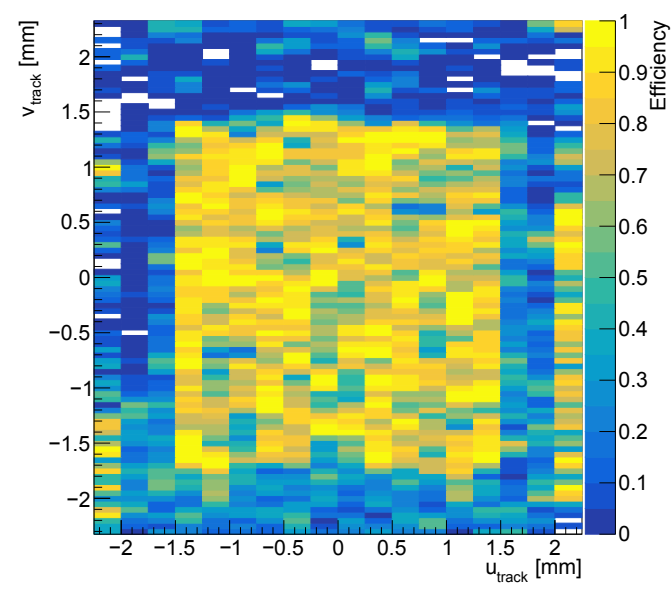

(a)

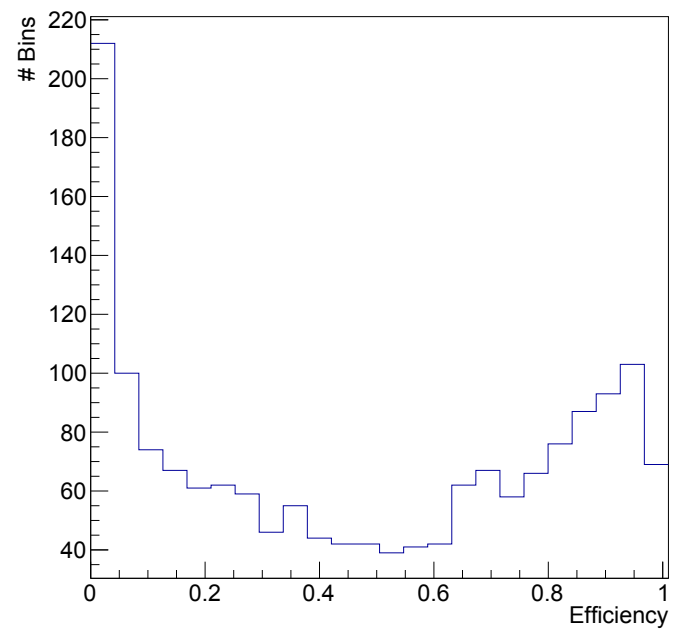

(c)

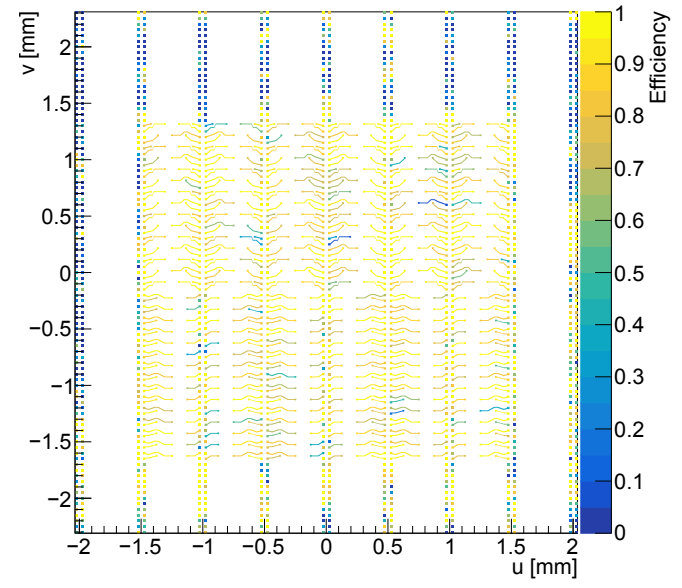

(b)

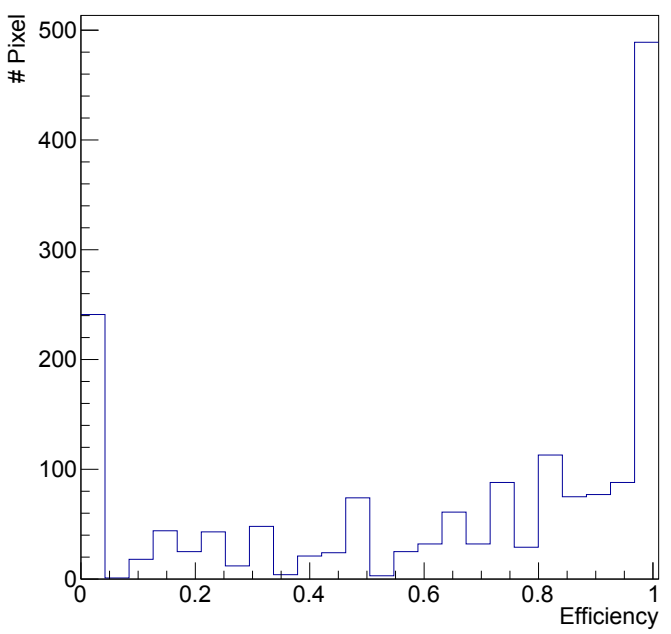

(d)

Figure 6.16.: (a): map of the efficiency for all expected tracks in the diamond, and in (b) map of the efficiency for expected tracks intersecting traces displayed in the read-out pixel column and row associated to the trace. The histograms summarize the content of the maps above. Diamond biased with $500 \mathrm{~V}$.

in the diamond, scratches on the surface, and metallisation defects are marked. The other category is dirt on the surface, number 1 . Regions with low efficiency that can be correlated to defects are circled in both maps. In almost all marked spots there is a dark spot in the diamond of varying size. The inefficiency circled in red and green, is due to two disabled pixels, which are connected with a big solder drop between the traces. There are more defects that do not reduce the efficiency, mostly scratches in the surface. Not for every inefficient area is a defect visually apparent.

The efficiency of the particle detection at the traces is discussed above with the cut 


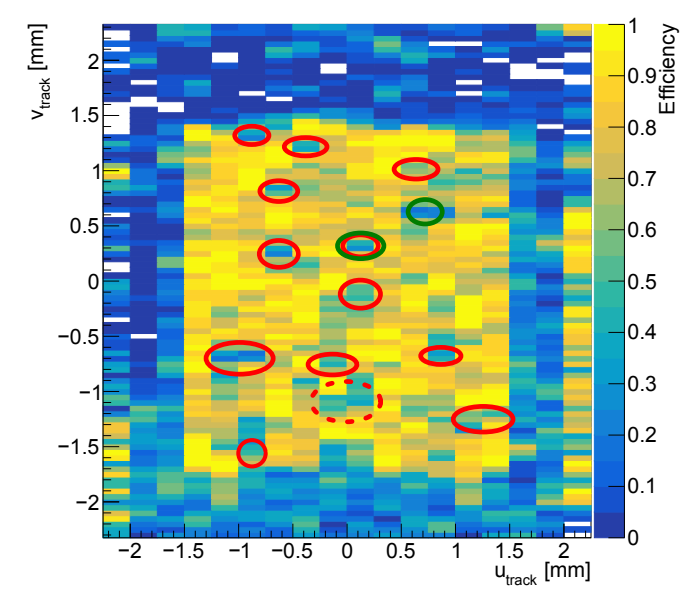

(a)

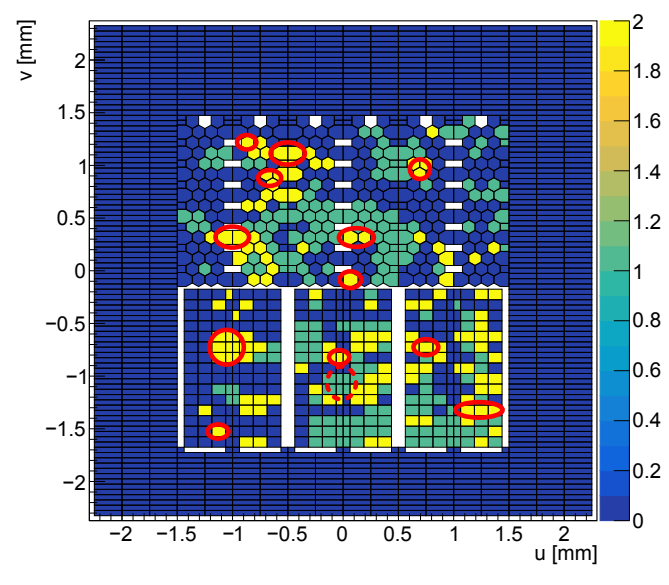

(b)

Figure 6.17.: (a): map of the efficiency at $500 \mathrm{~V}$ bias voltage. (b): map of defects (2) and dirt (1) for the planar diamond displayed in the layout of the bias grid. Correlated areas of low efficiency and defects are marked in red (dashed for dirt), green marks areas with disabled pixels.

on the track position. In Figure 6.18, the efficiency at the track position is projected into the rectangular and hexagonal super-cell and the traces overlayed for guidance. Because of the binning and the available number of tracks, the uncertainty per bin on the efficiency ranges between $3 \%$ to $10 \%$ depending on the data set. It is observed that near the bump bonds at the traces the efficiency is high and decreases with distance to the bump bond even at the trace. Between the traces coming from the left and the right, the efficiency is minimal along the $v$ direction. The width of this area is smallest with $(70 \pm 15) \mu \mathrm{m}$ where traces are close, and extends into areas without traces. As expected, the inefficient regions follow the global behaviour for different bias voltages, growing in case of lower global efficiency and vice versa. For $50 \mathrm{~V}$ bias voltage in most of the efficiency gap no particles at all are detected, while for $600 \mathrm{~V}$ the efficiency in the area is higher and it does not extend that far. The pixelation of the planar diamond detector is not optimal to detect particles with a high efficiency all over the pixel matrix. This is not unexpected because the metallisation is designed to connect the read-out pixels with the charge collecting electrodes in a $3 \mathrm{D}$ diamond detector.

The efficiency of the individual prototype pixel is displayed in Figure 6.19(a) for the bias voltages of $50 \mathrm{~V}, 200 \mathrm{~V}, 400 \mathrm{~V}$ and $500 \mathrm{~V}$, find the other data sets in Appendix A.2.2. For the calculation of the efficiency, only tracks intersecting the traces are used for the technical reason that only then the prototype pixel number is assigned to the track in the analysis framework. With this layout of small pixels not connected to each other, the assignment of a type to a track in the area between the pixels is not possible, so they are given the number -1 . The low efficiency for these tracks in this display is 


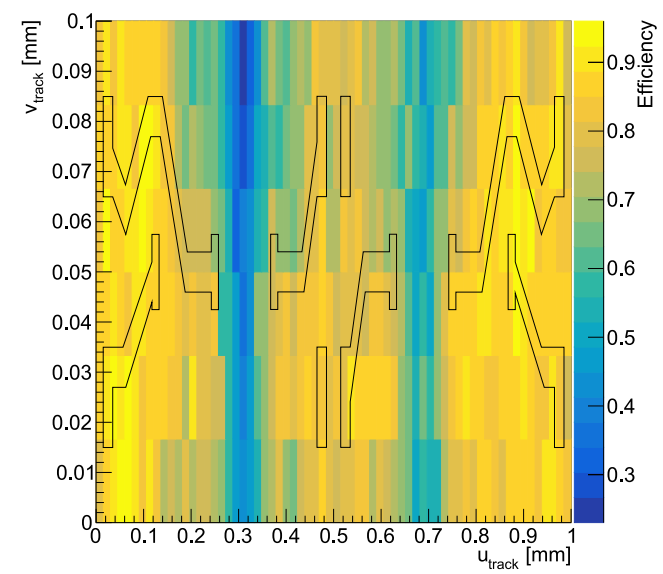

(a)

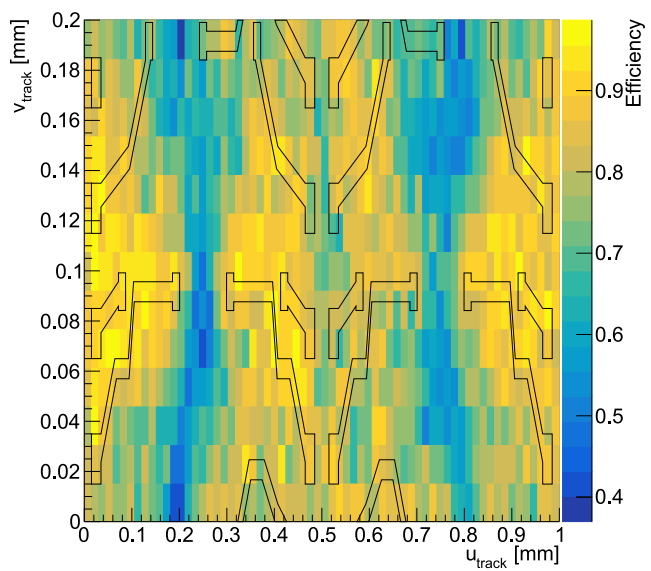

(b)

Figure 6.18.: Efficiency at the track position folded into the rectangular and hexagonal super-cell in (a) and (b), respectively. Bias voltage of $500 \mathrm{~V}$.

misleading because the different regions of the diamond are not differentiated. This is done in Figure 6.19(b) and shows that in the metallised area these tracks are more efficiently recorded for higher bias voltages. They are the core part of the matched tracks considering the small area of the diamond that is covered with metal.

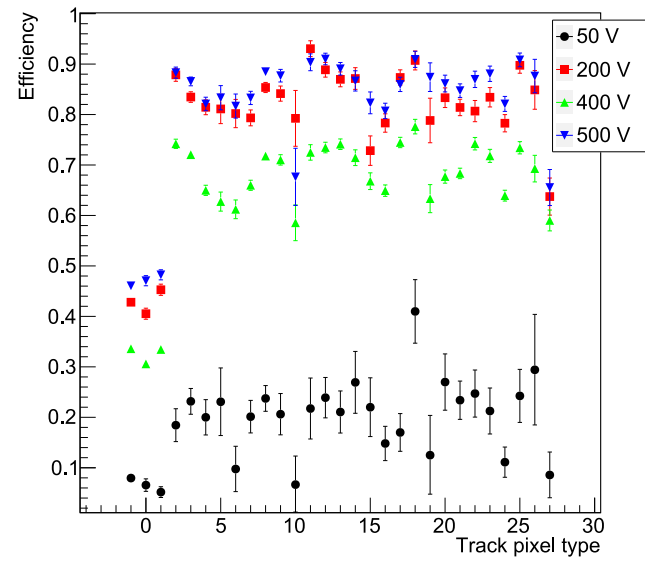

(a)

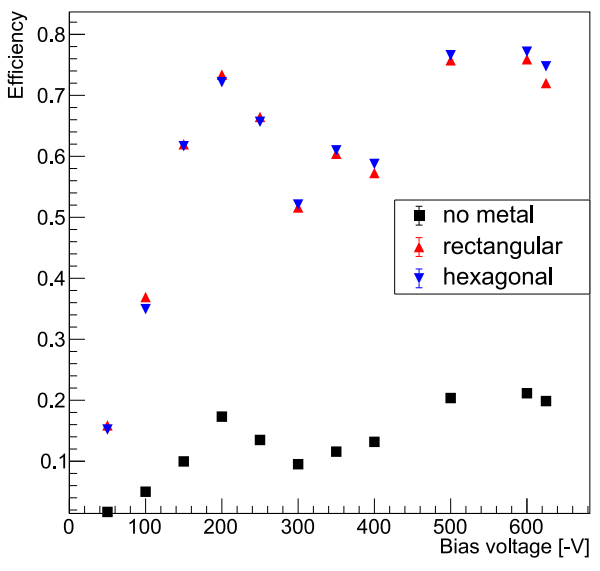

(b)

Figure 6.19.: (a): efficiency of the prototype pixels for track intersecting traces. (b): efficiency of tracks not intersecting traces, prototype pixel -1 . 


\section{Test Beam Measurements with Diamond Pixel Modules}

The particle detection with the bump bonds only, prototype pixels 0 and 1 , is not efficient as expected but improving with bias voltage. The comparison to the prototype pixels $5,6,10,19$, and 26 shows that already a small metal pad between the diamond and the bump bond doubles the efficiency compared to only bump bonds. The more efficient prototype pixels are the ones at the left and right edge of the super-cells. This can also be seen in the efficiency maps for the super-cells that in that area the efficiency is lower, and in the average charge maps the same for the collected charge. At the highest bias voltages this effect is reduced and the efficiency increases for these pixels. Perhaps the charges get better separated to the individual pixels.

The combination of the results from the track position maps, charge collection and the localised efficiencies shows that the traces can efficiently collect charges for a high enough electrical field applied. In a 3D diamond these traces are not the foreseen charge collection spots but the electrodes in the bulk. To connect the collecting electrodes the traces cross through other pixels. As the traces collect charges very localised some of the charges in a pixel could be recorded via the traces by a different read-out pixel. This migration leads to larger clusters or missing charge if the threshold is not crossed in the secondary pixels. With these studies of the trace efficiencies and simulations of the electrical fields in planar and 3D diamonds the influence of the parasitic charge collection for the 3D diamonds could be estimated.

\subsubsection{D Diamond}

In Figure 6.20(a), the intersection of matched tracks in the local coordinate system is displayed for the data set with the 3D diamond module at $60 \mathrm{~V}$ bias voltage. Over the whole diamond area only very few small regions, single pixels, have multiple track counts. The same behaviour is observed for the diamond at different bias voltages, see Appendix A.2.3. In Table 6.8, for the different measurements the statistical information about number of hits and tracks is listed. For all measurements, around $5 \%$ of the tracks pointing at the diamond volume could have been matched to a hit at maximum considering only the number of hits and tracks and not their relation.

In total too few hits are measured even considering the amount of pixels disabled due to noise beforehand. The very likely explanation is a too high discriminator threshold of the read-out chip. It is tuned to a target of $3000 \mathrm{e}$, as an attempt to suppress the noise in the diamond, see tuning results in Table 6.2. Apparently not only the noise is suppressed, but also the signal in most of the pixels is too small to cross the threshold. A measurement is performed with a ${ }^{90} \mathrm{Sr}$ source in the laboratory after the test beam with the same configuration, see Figure 6.20(b). The same pattern of pixels is observed that record hits. Because of time constraints this measurement was not done before the test beam. The measurements with radioactive sources with a threshold of 1500 e showed a more even distribution of hits in the diamond, compare Figure 6.6(a).

The efficiency per pixel is mapped to the layout in Figure 6.21 for the measurements with a bias voltage of $60 \mathrm{~V}$. The highest efficiency of a single pixel is around $70 \%$, the vast majority of the pixels have an efficiency below $5 \%$. The thresholds of the more efficient pixels are not lower than for the other pixels, which excludes a threshold fluctuation as 


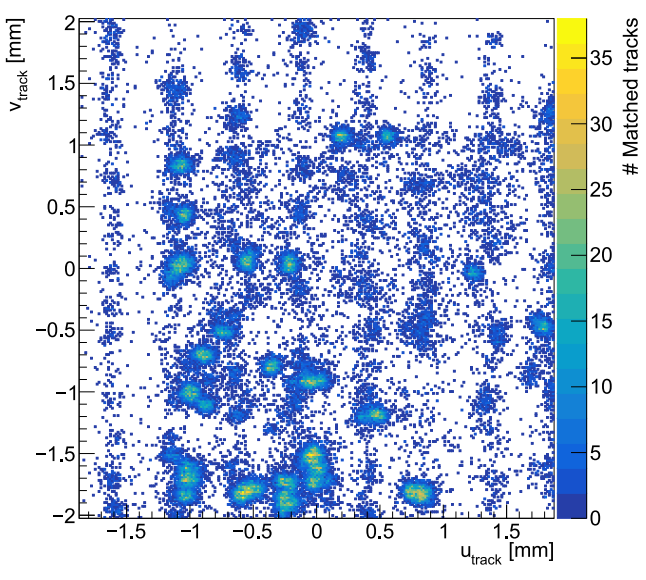

(a)

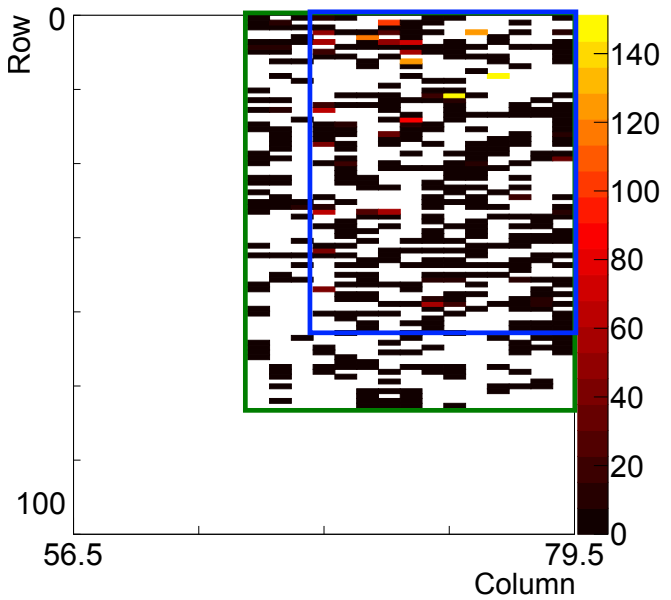

(b)

Figure 6.20.: (a): matched track incidents positions in the sensitive area of the $3 \mathrm{D}$ diamond module, biased with $60 \mathrm{~V}$. (b): ${ }^{90} \mathrm{Sr}$ source measurement as comparison. Both measurements with 3000 e threshold. Note the swapped row axis direction. Diamond area marked in green and metallised area in blue.

\begin{tabular}{|c|c|c|c|c|}
\hline Bias voltage [V] & Events [Mio] & Tracks on diamond [k] & Hits [k] & Hit/Tracks [\%] \\
\hline \hline 50 & 2.9 & $625(1)$ & $26.5(2)$ & 4.2 \\
\hline 60 & 12.1 & $2572(2)$ & $110.0(3)$ & 4.2 \\
\hline 70 & 1.5 & $324(1)$ & $16.2(2)$ & 4.9 \\
\hline
\end{tabular}

Table 6.8.: Statistics of the measurement with the 3D diamond.

explanation for the higher efficiency. A conclusive statement why these pixels are more efficient cannot be made. Most of these pixels have short trace connections and are close to the bump bond, but so are many more pixels with low efficiency.

From further analysis of the collected data with the 3D diamond module is abstained, because not the properties of the diamond sensor and the electrodes is observed, but only the misconfigured state of the read-out chip determines the behaviour. In follow up test beam measurements not part of this work, the read-out chip was configured with a special low noise tuning [76], which allowed observing an efficiency higher than $90 \%$ in working pixels which alomst includes the whole metallised area [77]. 
6. Test Beam Measurements with Diamond Pixel Modules

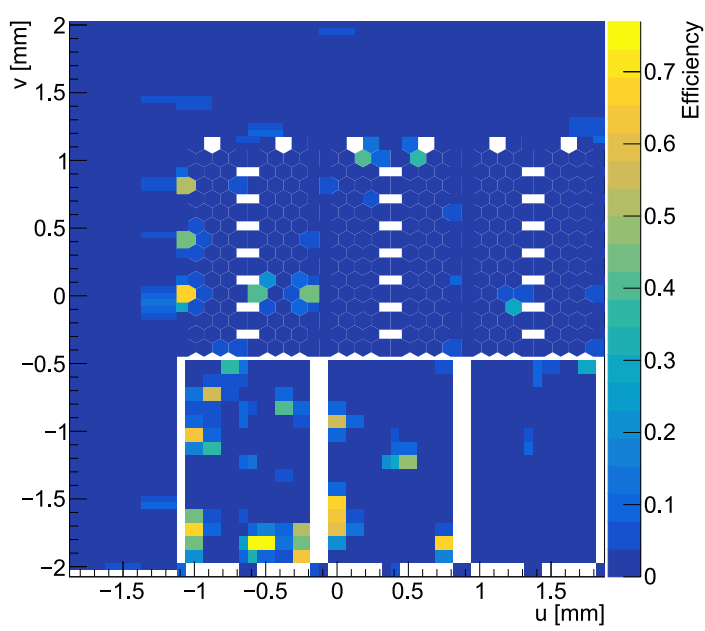

Figure 6.21.: Efficiency per pixel mapped to the layout of the 3D diamond module, biased with $60 \mathrm{~V}$. 
CHAPTER 7

\section{Conclusion and Outlook}

Tracking detectors in HEP experiments are crucial to resolve the origin of particles in collider experiments, allowing, e.g. to distinguish long lived particles like b quarks. In this thesis the production of such detectors is developed and investigated for the ATLAS detector and radiation hard diamond detectors studied for more future applications.

\subsection{Module Assembly and Testing for the ATLAS Upgrade}

The LHC will be upgraded to the HL-LHC in the near future. To be able to record tracking information with high precision, the inner detector of ATLAS will be replaced by the ITk. For the Pixel detector, 12000 modules will have to be assembled and tested according to high quality standards. In preparation for this effort, the processes are studied in the framework of the demonstrator project.

In this work, prototype tools for the assembly of the modules were developed and investigated for their production quality and the achievable alignment precision of the involved module parts. It could be shown that the production of the tools is possible to the needed precision, but between production batches can occure significant differences. The production can be further improved by using standardised feature dimensions and industrial standard parts. A limiting factor for the current production of tools was the choice of $3.4 \mathrm{~mm}$ diameter holes and matching pins, which is not a standard size and no precision tools were readily available to manufacture these. Despite this issue, the achieved alignment precision of the module parts with these tools were within the target tolerances of $50 \mu \mathrm{m}$ for lateral positioning. For this the matching of the positioning pins to the holes were of great importance.

During the production of five quad chip modules and four double chip modules, the assembly process was tested step by step. The findings were considered and solutions implemented in the newest version of the tools for the assembly of modules with the 


\section{Conclusion and Outlook}

RD53A chip. An extra jig for the flex will be used to allow for a controlled glue application to the flex with a stencil method. This enables the control of the amount of glue and the thickness of the glue layer, which is not possible in the current tool. The bare module will be aligned with fixed pins, not removable, which should increase the alignment precision compared to the previously used extrusion edges, because they are standardised parts and the position in the jig is easier to manufacture.

On a different level, the process chain can be improved by small tools, e.g. a holder for multiple flexes in the ultrasonic bath for the cleaning process. For the ITk production the number of workstations for the assembly needs to be scaled up from a single prototype station to multiple parallelised production stations to meet the production throughput and the time line. To ensure the continuous monitoring of the modules during the different production steps at different sites, a data base is under development, in which all data for a module from production to testing will be stored. Also for the storage and transportation of the assembled modules carrier boxes are designed with additional features to enable fast and safe connection of test systems to the module.

After the module assembly, the modules have to be qualified with a series of tests, ensuring mechanical and electrical properties. These test procedures are currently under development to standardise the quality control between the different testing sites, and to define quality criteria for the parameters under investigation. The as of now required tests are described in this thesis and some were carried out for the assembled prototype modules. Most of the modules performed to the expectation, but also some unexpected problems were observed like the misplacement of the read-out chips during bump bonding for one quad chip module.

To enable automation and paralellisation of the testing of multiple modules, a test stand was developed in the framework of this thesis. It consists of a cooling system, connections for power and read out of the modules, and capabilities for tests with radioactive sources. A prototype of the cooling system for one module has been installed and tested down to $-30{ }^{\circ} \mathrm{C}$ on the module, which exceeds the testing specifications. This test provides confidence that the cooling of more modules at more stations is feasible. These will be produced with the ITk module dimensions and read-out needs in mind. In the same step, the routing of the pipes and cables should be optimised for a better insulation of the cooling pipes and a clear distribution of cables to the modules.

The automation of the electrical tests is now also provided in hardware with the installation of a motorised holder for the radioactive source. This was one of the main missing pieces in the testing process, which relied on manual intervention. With this done, the next automation step will be to set up the test cycles and to upload the results to the module data base.

The modules produced in the scope of this thesis were integrated into the FE-I4 outer barrel demonstrator stave at CERN. There they provide valuable information for testing the system of a stave with serial powering and multi channel read-out. These are important tests leading to the building of the ITk. The module production tools were also distributed to other institutes, enabling the production of the double chip modules for the demonstrator. 
On the road to ITk production the next step is the module production with the RD53A prototype chip. For this the improved assembly tools will be used, which should not need further functional changes for the real production. With these modules the fine pitch bump bonding can be assessed and tested in thermal stress tests, which will be an important test for the final read-out chip and module composition. Also large scale system tests as currently done with the FE-I4 based modules are planned for the near future. All in all the project is moving fast in direction of production of the ITk starting in 2021.

\subsection{Test Beam Measurements with Diamond Pixel Modules}

Diamond has the potential as a good sensor material in high radiation environments. In the last years the fabrication of $3 \mathrm{D}$ electrodes inside the bulk of diamond with femtosecond laser has been intensively studied to increase and control the charge collection. Building pixelated hybrid detectors is the consequent next step.

The 3D diamond sensor bump bonded for the first time to an ATLAS FE-I4 chip investigated in this thesis exhibited a large amount of noisy pixels. A configuration of the read-out chip with a high threshold was chosen to counteract the noise, but this also suppressed the signal too much to record enough hits in the test beam measurements. A refined configuration with lower threshold was used later in the laboratory.

Instead a planar diamond detector with the same metallisation as pixels was investigated in test beam measurements. A maximum efficiency of $76 \%$ in the pixelated regions was observed. The regions between the pixels were systematically less efficient, which is expected because the metal traces were not designed as pixels but for the charge transport from the 3D electrode to the read-out chip. Therefore no full coverage of the diamond area with traces was needed. The results can be nevertheless used for future 3D diamond detectors to judge the influence of parasitic charge collection at the metal traces compared to the charge collection with the 3D electrodes.

The pixel geometry of the 3D and the planar detector does not consist of standard rectangular pixels but also hexagons and even more complicated structures in the case of the planar detector. For the analysis of the test beam data, a new pixel layout description in the TBSW framework was implemented. It allows for any polygonal shaped pixels in an arbitrary arrangement, making the layout description very flexible. As the example of the planar diamond detector showed, also very unusual pixel geometries can be successfully implemented and studied.

In the RD42 collaboration, there are more studies for 3D diamond detectors ongoing. Recently, measurements with CMS read-out chips observed efficiencies of $99.3 \%$ and with FE-I4 read-out chip of $97.8 \%$ [78]. In comparison to the 3D diamond under study in this thesis, the electrode diameter is reduced to $2.6 \mu \mathrm{m}$ and pixel cells of $50 \mu \mathrm{m} \times$ $50 \mu \mathrm{m}$ are implemented, multiple cells connected to a read-out pixel, simplifying the pixel matrix compared to the layout used for the diamonds in this thesis. In the future, the RD53A read-out chip will be used for direct pixel to pixel connections [79]. The radiation hardness of the $3 \mathrm{D}$ diamond detectors will be tested up to $10^{17}$ hadrons $/ \mathrm{cm}^{2}$ 


\section{Conclusion and Outlook}

to prove the capability as a tracking detector in the innermost layers of the detectors at the HL-LHC. Also, considerations are to produce detectors with $25 \mu \mathrm{m} \times 25 \mu \mathrm{m}$ pixels to potentially increase even further the radiation hardness. A goal is that $3 \mathrm{D}$ diamond detectors are ready to be considered for the replacement of the innermost layers during the HL-LHC phase. Also, for future planned accelerators like the Future Circular Collider (FCC) diamond detectors will have their spot, because the radiation levels will not decrease compared to the HL-LHC. Radiation hard detectors like diamond detectors will be required for tracking and for beam conditions monitoring. 
[1] F. Englert and R. Brout, Broken Symmetry and the Mass of Gauge Vector Mesons, Phys. Rev. Lett. 13 (1964) 321-323.

[2] P. W. Higgs, Broken Symmetries and the Masses of Gauge Bosons, Phys. Rev. Lett. 13 (1964) 508-509.

[3] ATLAS Collaboration, Observation of a new particle in the search for the Standard Model Higgs boson with the ATLAS detector at the LHC, Phys. Lett. B 716 (2012) 1-29.

[4] CMS Collaboration, Observation of a new boson at a mass of $125 \mathrm{GeV}$ with the CMS experiment at the LHC, Phys. Lett. B 716 (2012) 30-61.

[5] Super-Kamiokande Collaboration, Evidence for Oscillation of Atmospheric Neutrinos, Phys. Rev. Lett. 81 (1998) 1562-1567.

[6] ATLAS Collaboration, Standard Model Summary Plots Summer 2019, Tech. Rep. ATL-PHYS-PUB-2019-024, 2019.

[7] F. Zwicky, Die Rotverschiebung von extragalaktischen Nebeln, Helv. Phys. Acta 6 (1933).

[8] F. Zwicky, On the Masses of Nebulae and of Clusters of Nebulae, ApJ 86 (1937) 217.

[9] V. C. Rubin and W. K. Ford, Rotation of the Andromeda Nebula from a Spectroscopic Survey of Emission Regions, ApJ 159 (1970) 379.

[10] Planck Collaboration, Planck 2013 results. XVI. Cosmological parameters, A\&A 571 (2014) A16.

[11] L. Evans and P. Bryant, LHC Machine, JINST 3 (2008) S08001. 


\section{BIBLIOGRAPHY}

[12] ALICE Collaboration, The ALICE experiment at the CERN LHC, JINST 3 (2008) S08002.

[13] ATLAS Collaboration, The ATLAS Experiment at the CERN Large Hadron Collider, JINST 3 (2008) S08003.

[14] CMS Collaboration, The CMS experiment at the CERN LHC, JINST 3 (2008) S08004.

[15] LHCb Collaboration, The LHCb Detector at the LHC, JINST 3 (2008) S08005.

[16] ATLAS Collaboration, ATLAS Insertable B-Layer Technical Design Report, Tech. Rep. CERN-LHCC-2010-013, ATLAS-TDR-19, 2010.

[17] G. Apollinari et al., High-Luminosity Large Hadron Collider (HL-LHC): Technical Design Report V. 0.1. CERN Yellow Reports: Monographs. CERN, 2017.

[18] H. Damerau et al., LHC Injectors Upgrade, Technical Design Report, Vol. I: Protons, Tech. Rep. CERN-ACC-2014-0337, 2014.

[19] J. Coupard et al., LHC Injectors Upgrade, Technical Design Report, Vol. II: Ions, Tech. Rep. CERN-ACC-2016-0041, 2016.

[20] H. C. Beck, Module development for the HL-LHC ATLAS ITk Pixel upgrade, JINST 14 (2019) C12008.

[21] ATLAS Collaboration, Technical Design Report for the ATLAS Inner Tracker Pixel Detector, Tech. Rep. CERN-LHCC-2017-021, ATLAS-TDR-030, 2017.

[22] ATLAS Collaboration, Expected Tracking Performance of the ATLAS Inner Tracker at the HL-LHC, Tech. Rep. ATL-PHYS-PUB-2019-014, 2019.

[23] RD53 Collaboration, RD Collaboration Proposal: Development of pixel readout integrated circuits for extreme rate and radiation, Tech. Rep.

CERN-LHCC-2013-008, LHCC-P-006, 2013.

[24] RD53 Collaboration, RD53A Integrated Circuit Specifications, Tech. Rep. CERN-RD53-PUB-15-001, 2015.

[25] S. Kühn, Results of prototyping for the Phase-II upgrade of the pixel detector of the ATLAS experiment, JINST 14 (2019) C04010.

[26] P. Moreira et al., The GBT-SerDes ASIC prototype, JINST 5 (2010) C11022.

[27] E. Buschmann, ATLAS Phase-II-Upgrade Pixel Demonstrator Read-out, PoS TWEPP2018 (2019) 046.

[28] N. Lehmann, Control and Monitoring for a serially powered pixel demonstrator for the ATLAS Phase II upgrade, PoS TWEPP2018 (2019) 133. 
[29] H. C. Beck, TCT measurements with diamond for tracking detectors at high energy experiments, Master's thesis, Georg-August Universität Göttingen, 2015. II. Physik-UniGö-MSc-2015/05.

[30] W. R. Leo, Techniques for Nuclear and Particle Physics Experiments: A How-to Approach. Springer-Verlag, 1994.

[31] M. Tanabashi et al. (Particle Data Group), Review of Particle Physics, Phys. Rev. D 98 (2018) 030001.

[32] L. Landau, On the energy loss of fast particles by ionization, J. Phys.(USSR) 8 (1944) 201-205.

[33] P. V. Vavilov, Ionization losses of high-energy heavy particles, Sov. Phys. JETP 5 (1957) 749-751.

[34] H. Bichsel, Straggling in Thin Silicon Detectors, Rev. Mod. Phys. 60 (1988) 663-699.

[35] W. Shockley, Currents to Conductors Induced by a Moving Point Charge, J. Appl. Phys. 9 (1938) 635-636.

[36] S. Ramo, Currents Induced by Electron Motion, Proceedings of the IRE 27 (1939) $584-585$.

[37] I. Peric, Active pixel sensors in high-voltage CMOS technologies for ATLAS, JINST 7 (2012) C08002.

[38] A. L. Schorlemmer, Monitoring Radiation Damage in the ATLAS Pixel Detector. $\mathrm{PhD}$ thesis, Georg-August Universität Göttingen, 2014.

II.Physik-UniGö-Diss-2014/03.

[39] G. Kramberger, Signal development in irradiated silicon detectors. PhD thesis, University Ljubljana, 2001. CERN-Thesis-2001-038.

[40] W. de Boer et al., Radiation hardness of diamond and silicon sensors compared, physica status solidi (a) 204 (2007) 3004-3010.

[41] L. Graber, Radiation Hard 3D Diamond Sensors for Vertex Detectors at HL-LHC. $\mathrm{PhD}$ thesis, Georg-August Universität Göttingen, 2016.

II. Physik-UniGö-Diss-2015/04.

[42] H. Pernegger, High Mobility Diamonds and Particle Detectors. WILEY-VCH, 2008.

[43] H. Pernegger et al., Charge-carrier properties in synthetic single-crystal diamond measured with the transient-current technique, J. Appl. Phys. 97 (2005) 073704. 


\section{BIBLIOGRAPHY}

[44] I. Friel, Optical Engineering of Diamond, ch. 2: Optical Quality Diamond Grown by Chemical Vapor Deposition. WILEY-VCH, 2013.

[45] F. P. Bundy et al., Man-made diamonds, Nature 176 (1955) 51-55.

[46] Y. Zhang et al., Grain Boundary Structure and Growth Sequence of Diamond Thin Film, Materials Science Forum 204-206 (1996) 207.

[47] D. Asner et al., Diamond pixel modules, Nucl. Instr. and Meth. A 636 (2011) $125-129$.

[48] S. I. Parker et al., 3D - A proposed new architecture for solid-state radiation detectors, Nuc. Instr. and Meth. A 395 (1997) 328-343.

[49] T. Kononenko et al., Microstructuring of diamond bulk by IR femtosecond laser pulses, Appl. Phys. A 90 (2008) 645-651.

[50] V. Konov, Laser in micro and nanoprocessing of diamond materials, Laser Photonics Rev. 6 (2012) 739-766.

[51] G. Mainfray and G. Manus, Multiphoton ionization of atoms, REP PROG PHYS 54 (1991) 1333-1372.

[52] V. I. Konov et al., Optical Engineering of Diamond, ch. 12: Laser Micro- and Nanoprocessing of Diamond Materials. WILEY-VCH, 2013.

[53] M. Neff et al., Femtosecond laser writing of buried graphitic structures in bulk diamond, Appl. Phys. A 97 (2009) 543.

[54] A. Jesacher and M. J. Booth, Parallel direct laser writing in three dimensions with spatially dependent aberration correction, Opt. Express 18 (2010) 21090-21099.

[55] B. Sun, P. S. Salter, and M. J. Booth, High conductivity micro-wires in diamond following arbitrary paths, Appl. Phys. Lett. 105 (2014).

[56] M. Garcia-Sciveres et al., The FE-I4 pixel readout integrated circuit, Nucl. Instr. Meth. A 636 (2011) S155-S159.

[57] FE-I4 Collaboration, The FE-I4B Integrated Circuit Guide, Version 2.3 (2012).

[58] B. Abbott et al., Production and integration of the ATLAS Insertable B-Layer, JINST 13 (2018) T05008.

[59] M. Backhaus et al., Development of a versatile and modular test system for ATLAS hybrid pixel detectors, Nucl. Instr. Meth. A 650 (2011) 37-40.

[60] E. Buschmann, Development with the upgraded USBPix Test System for the ATLAS Pixel Front-end Electronics, Master's thesis, Georg-August Universität Göttingen, 2016. 
[61] D. Sonntag, Important new Values of the Physical Constants of 1986, Vapour Pressure Formulations based on ITS-90, and Psychrometer Formulae, Meteorol. Z. 40 (1990).

[62] T. Hüser, Module tests for the ATLAS pixel detector, Bachelor's thesis, Georg-August Universität Göttingen, 2018. II. Physik-UniGö-BSc-2018/01.

[63] M. H. Nazaré, B. Foster, and R. S. Gilmore, Development of diamond tracking detectors for high luminosity experiments at the $L H C$, Tech. Rep. CERN-DRDC-94-21, DRDC-P-56, 1994. Over title: R\&D proposal.

[64] R. Diener et al., The DESY II test beam facility, Nucl. Instr. and Meth. A 922 (2019) 265-286.

[65] H. Jansen et al., Performance of the EUDET-type beam telescopes, EPJ Tech. Instrum. 3 (2016) 7.

[66] C. Hu-Guo et al., First reticule size MAPS with digital output and integrated zero suppression for the EUDET-JRA1 beam telescope, Nucl. Instr. and Meth. A 623 (2010) 480-482.

[67] D. Cussans, Description of the JRA1 trigger logic unit (TLU), v0. 2c, Tech. Rep. EUDETMemo-2009-04, 2009.

[68] P. Ahlburg et al., EUDAQ - A Data Acquisition Software Framework for Common Beam Telescopes, Tech. Rep. arXiv:1909.13725, 2019.

[69] H. Kagan. Personal communication.

[70] T. Bisanz, Test beam studies of pixel detector prototypes for the ATLAS-Experiment at the High Luminosity Large Hadron Collider. PhD thesis, Georg-August Universität Göttingen, 2019. CERN-Thesis-2018-444, II.Physik-UniGö-Diss-2018/03.

[71] M. Schwickardi, Charakterisierung neuer Pixeldetektoren, Bachelor's thesis, Georg-August Universität Göttingen, 2016. II. Physik-UniGö-BSc-2016/05.

[72] R. Brun and F. Rademakers, ROOT: An object oriented data analysis framework, Nucl. Instr. and Meth. A 389 (1997) 81-86.

[73] B. Schwenker, Development and validation of a model for the response of the Belle II vertex detector. $\mathrm{PhD}$ thesis, Georg-August Universität Göttingen, 2014. CERN-THESIS-2014-165, II. Physik-UniGö-Diss-2014/05.

[74] U. Stolzenberg, Radiation length measurements with high-resolution telescopes. $\mathrm{PhD}$ thesis, Georg-August Universität Göttingen, 2019. II.

Physik-UniGö-Diss-2019/06. 


\section{BIBLIOGRAPHY}

[75] R. Frühwirth, Application of Kalman filtering to track and vertex fitting, Nucl. Instr. and Meth. A 262 (1987) 444-450.

[76] J. Janssen, Test beam results of ATLAS DBM pCVD diamond detectors using a novel threshold tuning method, JINST 12 (2017) C03072.

[77] J. Janssen. Personal communication.

[78] M. Reichmann et al., New test beam results of $3 D$ and pad detectors constructed with poly-crystalline CVD diamond, Nucl. Instr. and Meth. A (2019) 162675.

[79] H. Kagan and W. Trischuk, Development of Diamond Tracking Detectors for High Luminosity Experiments at the LHC, HL-LHC and Beyond, Tech. Rep.

CERN-LHCC-2018-015, LHCC-SR-005, 2018. 


\section{Acknowledgements}

First and foremost I want to thank Arnulf Quadt for giving me the opportunity to graduate and conduct the research in his group. Many thanks for sticking with me and pushing me on when I wanted to give up.

A very special thank goes to Jens Weingarten, Jörn Lange, and Jörn Große-Knetter for their support in every situation, for scientific and all other advices. For the good working atmosphere and the helping hands I want to thank first the hardware people: Tobias Bisanz, Eric Buschmann, Ali Skaf, Silke Möbius, Rafael Goncalves Gama, Julia Rieger, Lars Graber and the before mentioned trio. But in the same way I want to thank all colleagues in the institute. A special thank is for the electronics and mechanics workshop in the institute and the faculty, without their help many things would not have been possible. Especially I want to mention Andreas König, may you be remembered as friendly and funny as you were, and Tim Kanngießer.

One not simply does test beams, may or may not be a saying, but with the help of Jens Weingarten and Tobias Bisanz it becomes a lot simpler. Also I want to thank the ITk Pixel test beam crew for additional help and letting me put some diamond detectors in their beam. All the recorded data would have been of no use, if not for Benjamin Schwenker helping with the analysis of the data and the peculiar pixel layouts.

For enduring the first versions of this thesis and improving it with their comments, I have to thank Silke Möbius, Ali Skaf, Jörn Große-Knetter, and Jörn Lange.

Last and definitely not least, I want to thank my family and friends for always being there for me, even in their difficult times. Thank you, Désirée Renz, for your support.

Some of the measurements leading to these results have been performed at the Test Beam Facility at DESY Hamburg (Germany), a member of the Helmholtz Association (HGF). 

Appendices 

APPENDIX $\mathrm{A}$

Appendix

\section{A.1. Module Testing}

\section{A.1.1. IV Sensor Characteristic}

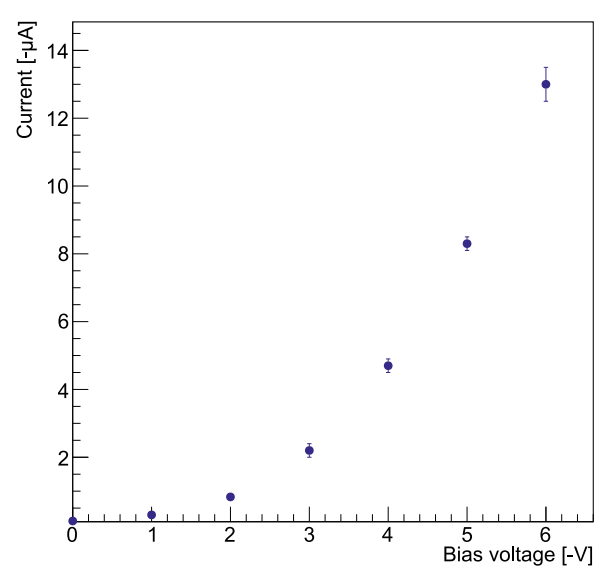

(a)

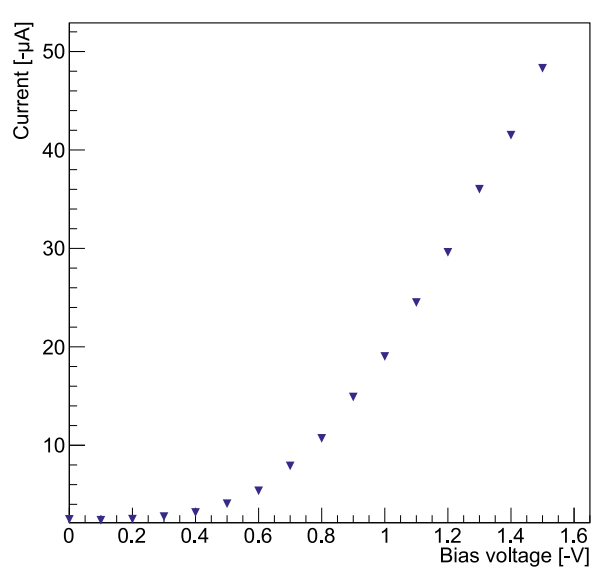

(b)

Figure A.1.: IV sensor measurements for module QSi9 and DSi15 in (a) and (b), respectively.

\section{A.1.2. Pixel Quality}




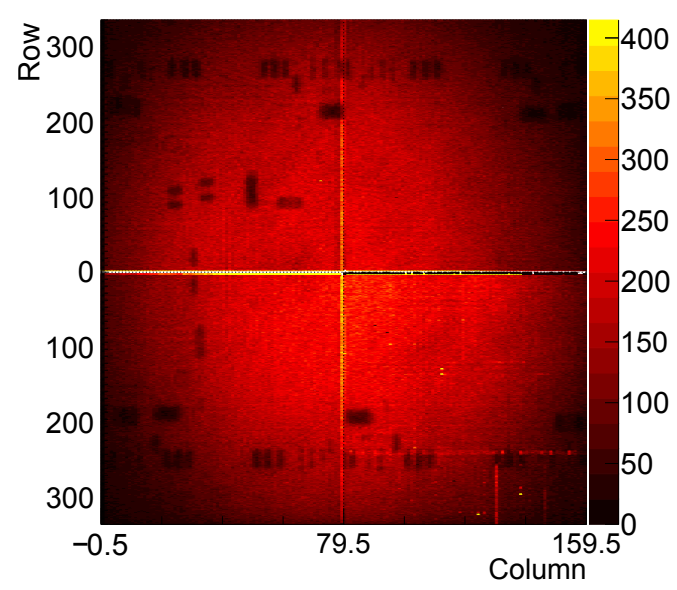

(a)

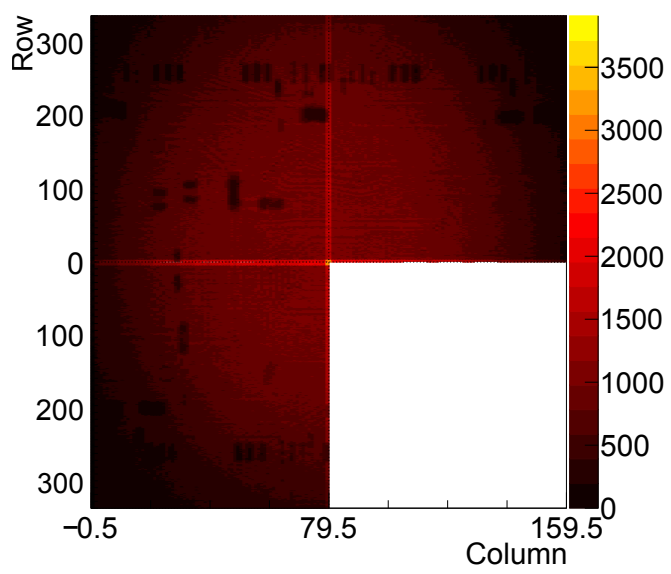

(b)

Figure A.2.: Occupancy map of an ${ }^{90} \mathrm{Sr}$ source measurement for 15 min with module QSi9 in (a) and the same for module QSi11 for $8 \mathrm{~min}$ in (b) as reference.

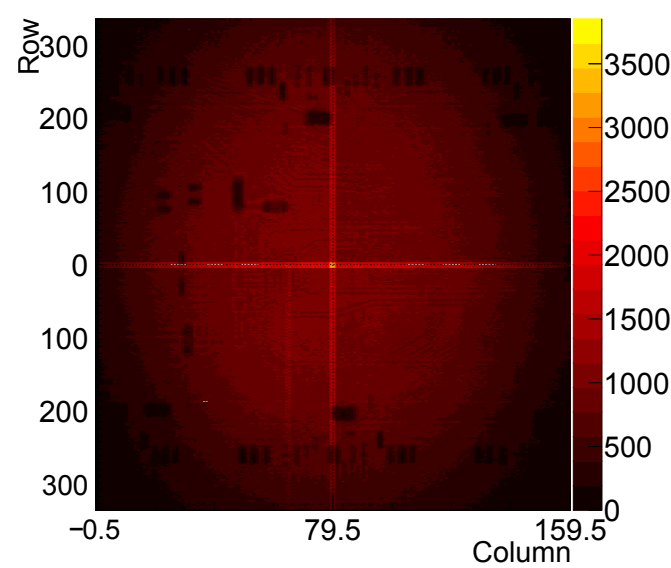

(a)

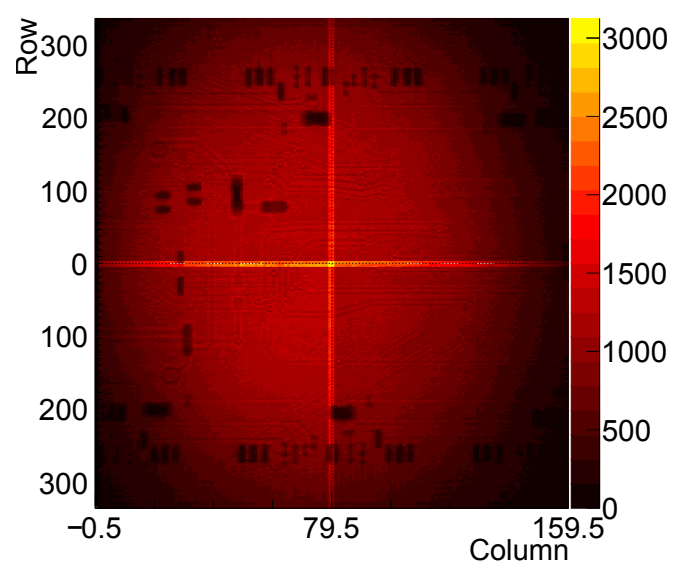

(b)

Figure A.3.: Occupancy map of an ${ }^{90} \mathrm{Sr}$ source measurement for 8 min with module QSi12 in (a) and the same for module QSi27 for 15 min in (b) as reference. 


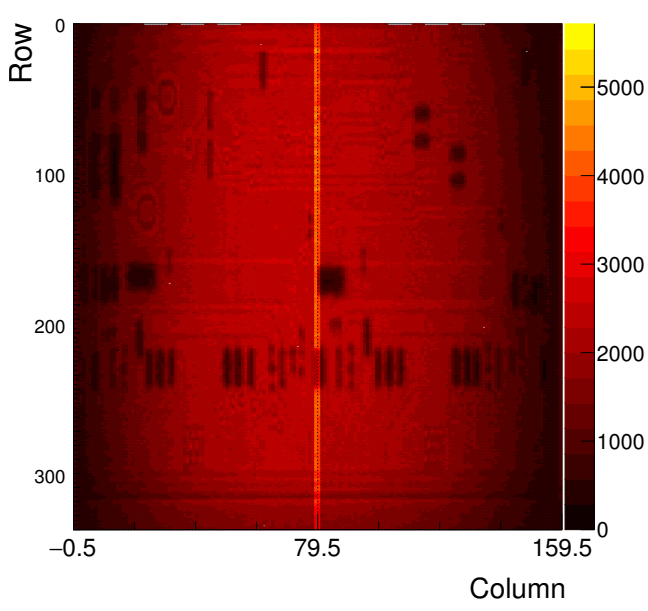

(a)

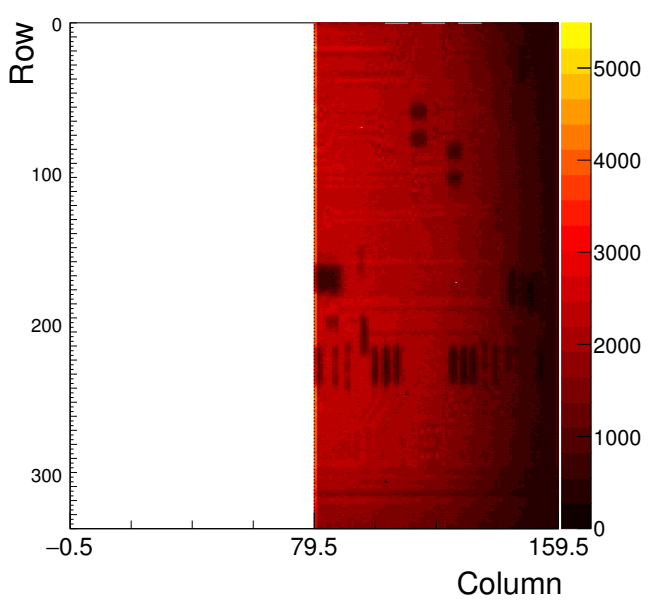

(b)

Figure A.4.: Occupancy map of an ${ }^{90} \mathrm{Sr}$ source measurement for $15 \mathrm{~min}$ with module DSi12 in (a) and the same for module DSi13 for $15 \mathrm{~min}$ in (b) as reference.

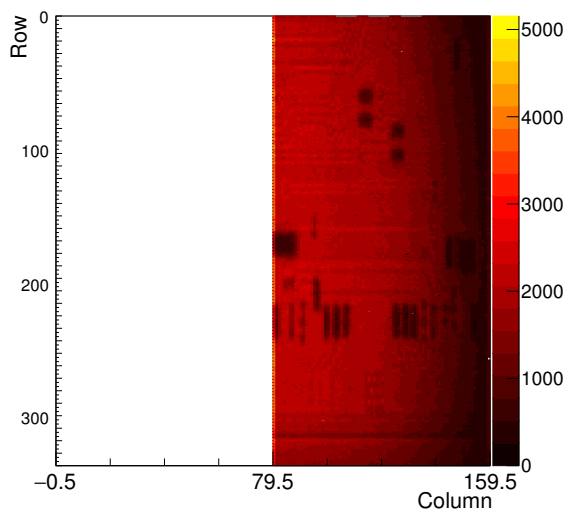

Figure A.5.: Occupancy map of an ${ }^{90} \mathrm{Sr}$ source measurement for $15 \mathrm{~min}$ with module DSi14. 


\section{A. Appendix}

\section{A.1.3. Cooling System}

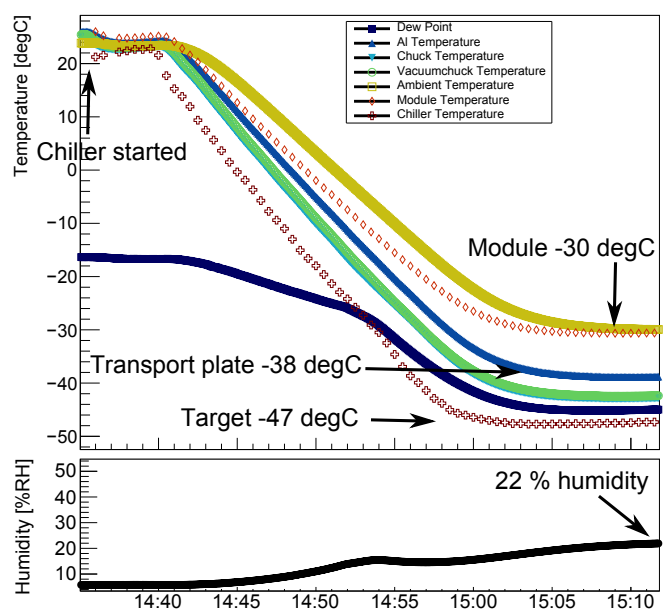

Figure A.6.: Cool down in the prototype test stand with the module QSi27.

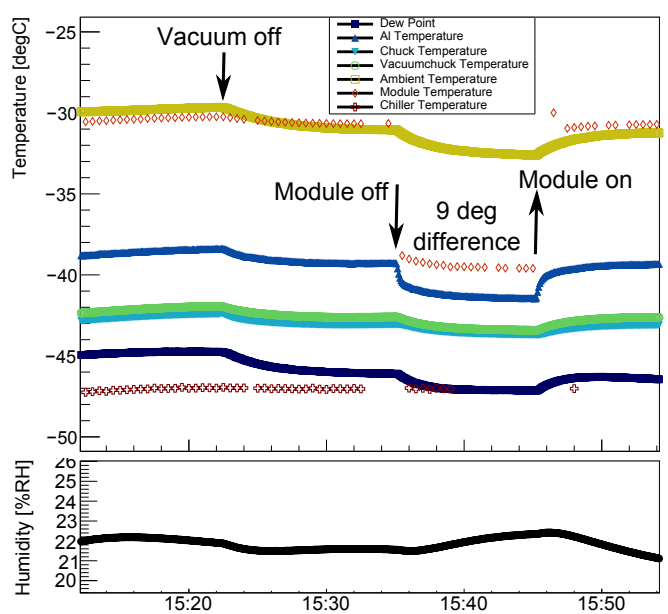

Figure A.7.: At stable cold temperature in the prototype test stand with the module QSi27. 


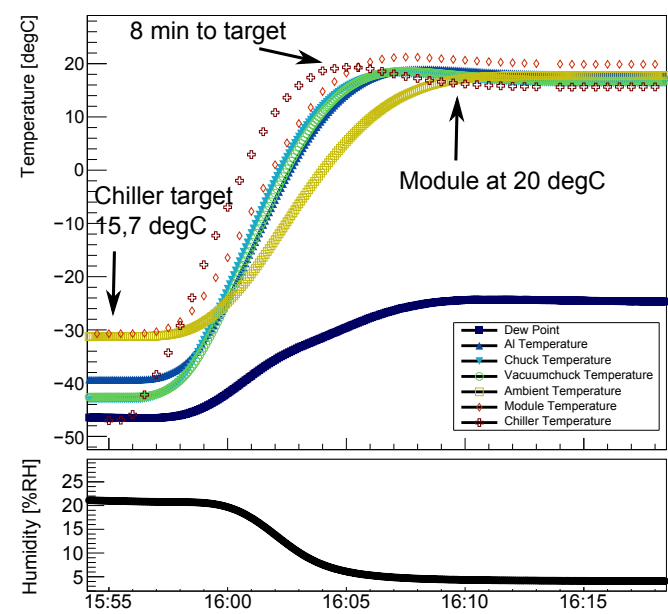

Figure A.8.: Warming up in the prototype test stand with the module QSi27.

\section{A.2. Test Beam Measurements with Diamond Pixel Detectors}

\section{A.2.1. Experimental Setup}

\begin{tabular}{|l|c|c|c|}
\hline Batch & Planar diamond, 1-3 & Planar diamond, 4 & 3D diamond \\
\hline \hline Telescope & DATURA & DATURA & DURANTA \\
\hline Plane 0 $[\mathrm{cm}]$ & 0.0 & 0.0 & 0.0 \\
\hline Plane $1[\mathrm{~cm}]$ & 15.0 & 14.8 & 14.9 \\
\hline Plane 2 $[\mathrm{cm}]$ & 30.0 & 30.6 & 29.9 \\
\hline Diamond $[\mathrm{cm}]$ & 40.3 & 41.0 & 45.1 \\
\hline Reference $[\mathrm{cm}]$ & 49.4 & 49.1 & 48.9 \\
\hline Plane 3 $[\mathrm{cm}]$ & 65.3 & 60.9 & 59.3 \\
\hline Plane $4[\mathrm{~cm}]$ & 74.3 & 75.8 & 74.1 \\
\hline Plane $5[\mathrm{~cm}]$ & 89.8 & 91.0 & 89.1 \\
\hline
\end{tabular}

Table A.1.: Setup of the telescope planes, distance to the first plane in beam, for the different measurement times.

\section{A.2.2. Planar diamond}

\section{Matched Track Positions}




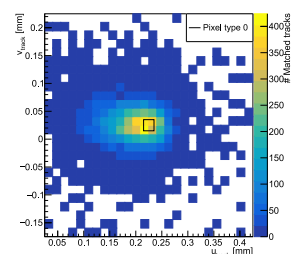

(a)

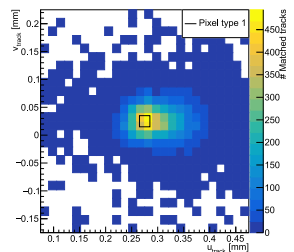

(b)

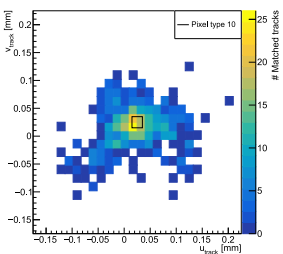

(c)

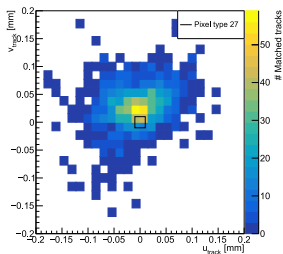

(d)

Figure A.9.: Map of matched track positions with hit in read-out connected to prototype pixel $0,1,10,27$ (left to right), at $500 \mathrm{~V}$.

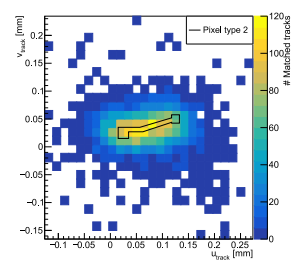

(a)

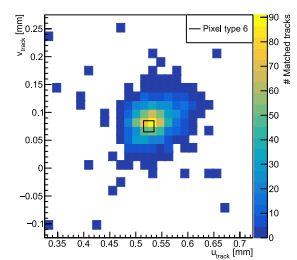

(e)

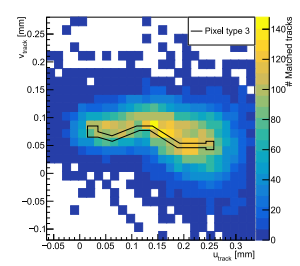

(b)

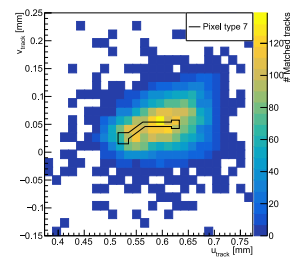

(f)

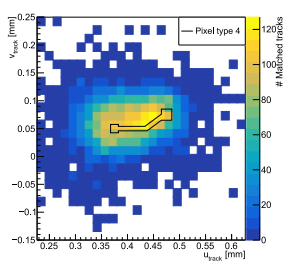

(c)

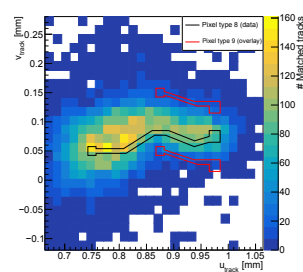

(g)

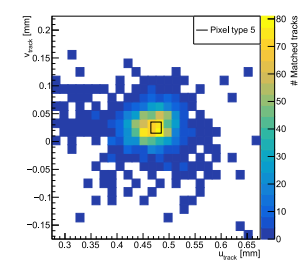

(d)

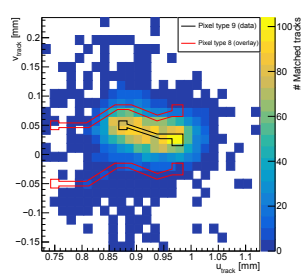

(h)

Figure A.10.: Map of matched track positions with hit in read-out connected to prototype pixel 2, 3, 4, 5 (first row), and 6, 7, 8, 9 (second row), at $500 \mathrm{~V}$. 


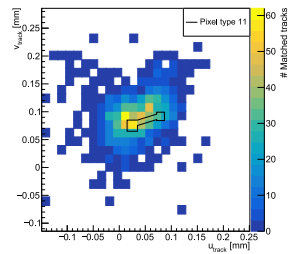

(a)

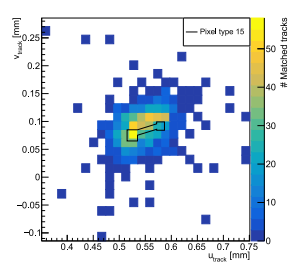

(e)

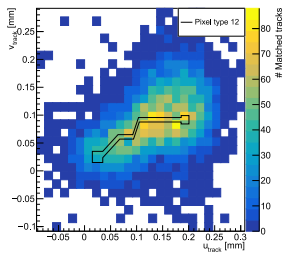

(b)

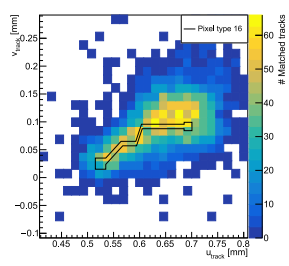

(f)

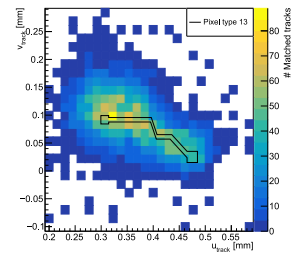

(c)

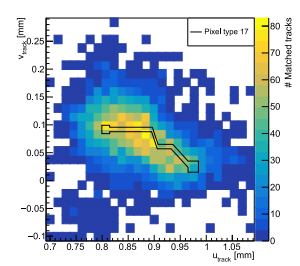

(g)

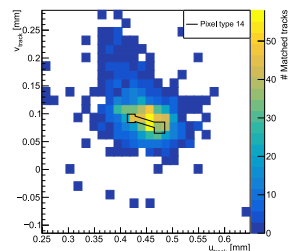

(d)

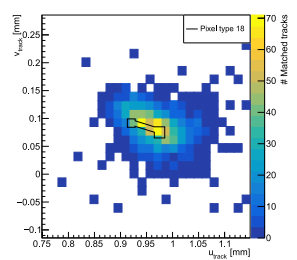

(h)

Figure A.11.: Map of matched track positions with hit in read-out connected to prototype pixel 11, 12, 13, 14 (first row), and 15, 16, 17, 18 (second row), at $500 \mathrm{~V}$.

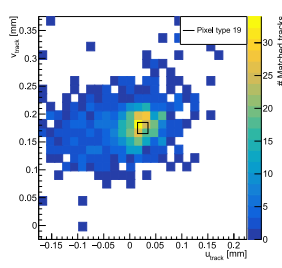

(a)

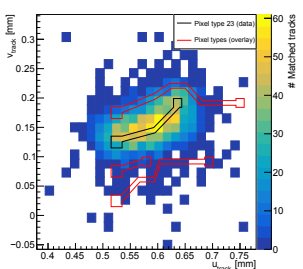

(e)

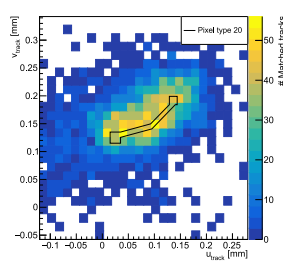

(b)

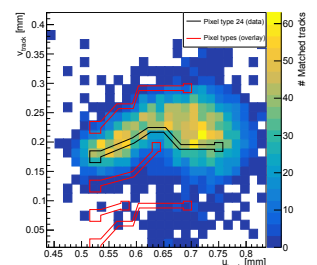

(f)

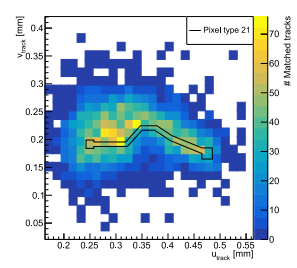

(c)

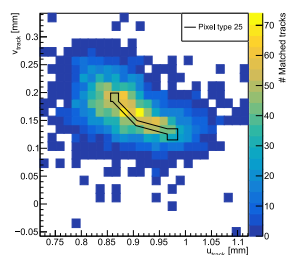

(g)

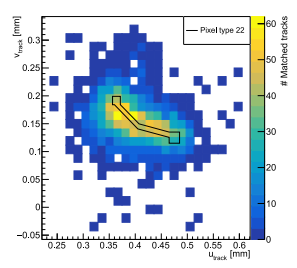

(d)

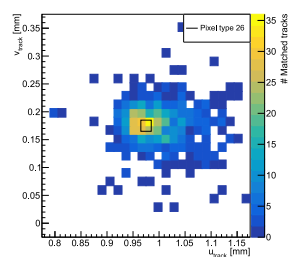

(h)

Figure A.12.: Map of matched track positions with hit in read-out connected to prototype pixel 19, 20, 21, 22 (first row), and 23, 24, 25, 26 (second row), at $500 \mathrm{~V}$. 


\section{A. Appendix}

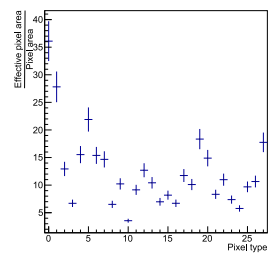

(a)

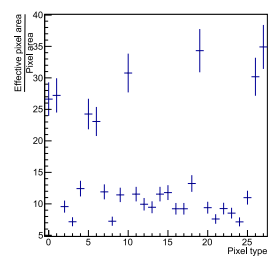

(e)

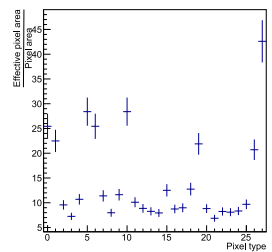

(b)

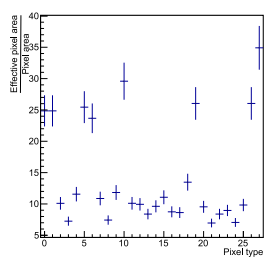

(f)

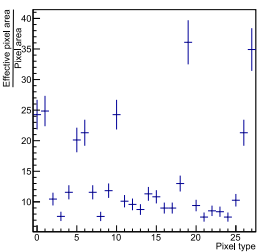

(c)

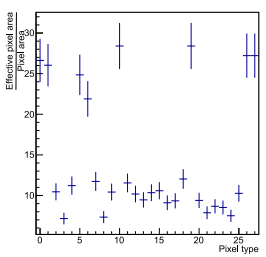

$(\mathrm{g})$

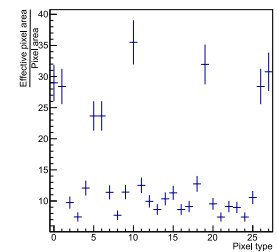

(d)

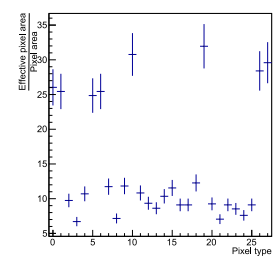

(h)

Figure A.13.: Ratio of the effective pixel area to the pixel area defined by the trace for the prototype pixels, at $50 \mathrm{~V}, 100 \mathrm{~V}, 150 \mathrm{~V}, 200 \mathrm{~V}$ (first row), and $250 \mathrm{~V}$, $300 \mathrm{~V}, 350 \mathrm{~V}, 400 \mathrm{~V}$ (second row).

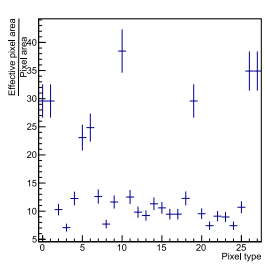

(a)

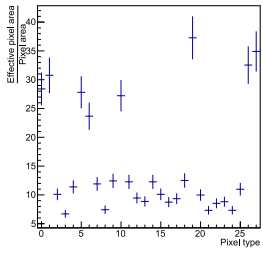

(b)

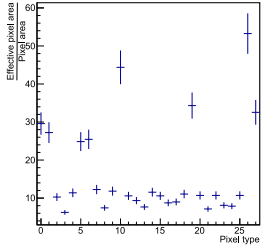

(c)

Figure A.14.: Ratio of the effective pixel area to the pixel area defined by the trace for the prototype pixels, at $500 \mathrm{~V}, 600 \mathrm{~V}$ with beam energy $4.0 \mathrm{GeV}, 600 \mathrm{~V}$ with beam energy $4.6 \mathrm{GeV}$ (left to right). 


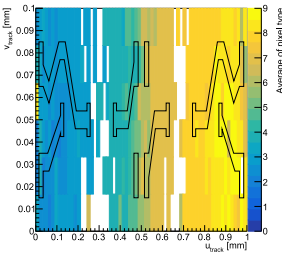

(a)

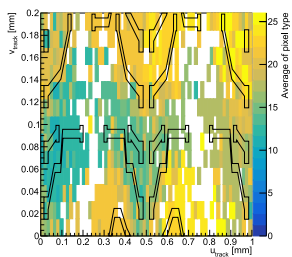

(e)

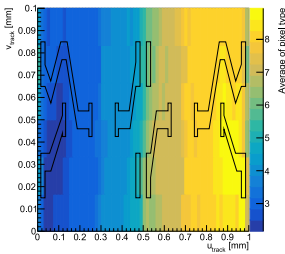

(b)

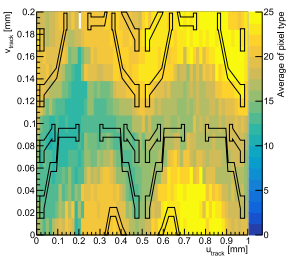

(f)

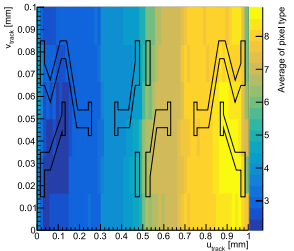

(c)

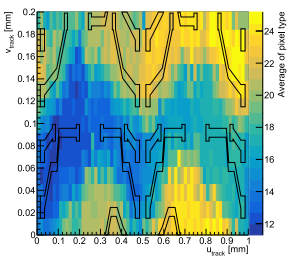

$(\mathrm{g})$

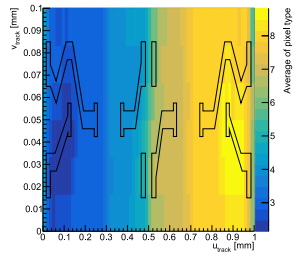

(d)

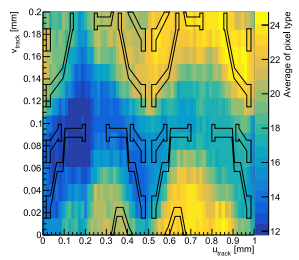

(h)

Figure A.15.: Average prototype pixel number of the hit pixel at the position of the matched track folded into the rectangular (first row) and hexagonal (second row) super-cell, at $50 \mathrm{~V}, 100 \mathrm{~V}, 150 \mathrm{~V}, 200 \mathrm{~V}$ (left to right).

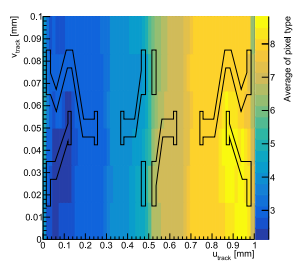

(a)

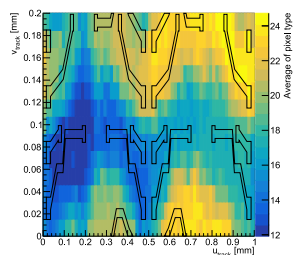

(e)

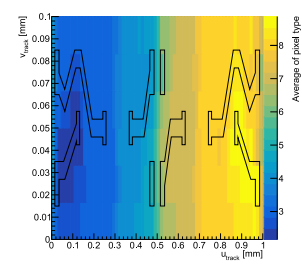

(b)

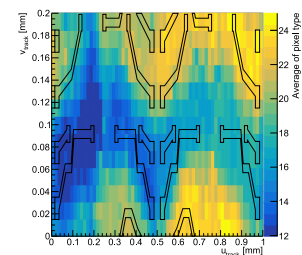

(f)

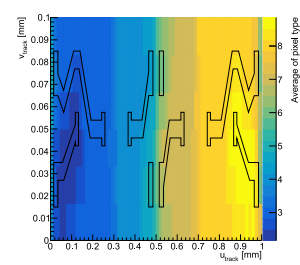

(c)

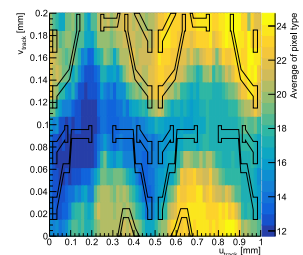

(g)

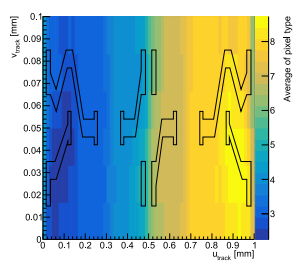

(d)

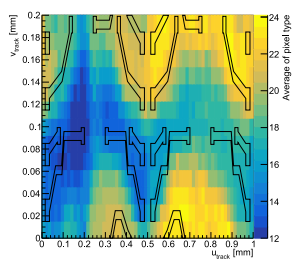

(h)

Figure A.16.: Average prototype pixel number of the hit pixel at the position of the matched track folded into the rectangular (first row) and hexagonal (second row) super-cell, at $250 \mathrm{~V}, 300 \mathrm{~V}, 350 \mathrm{~V}, 400 \mathrm{~V}$ (left to right). 


\section{A. Appendix}

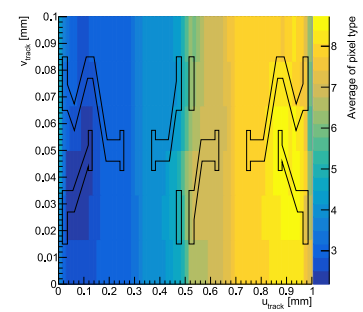

(a)

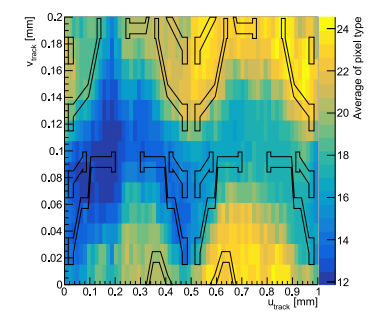

(d)

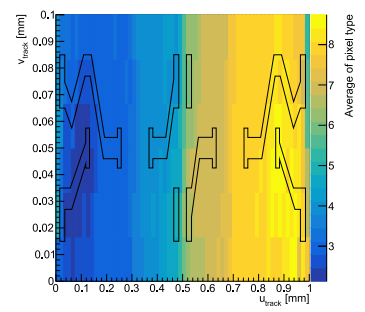

(b)

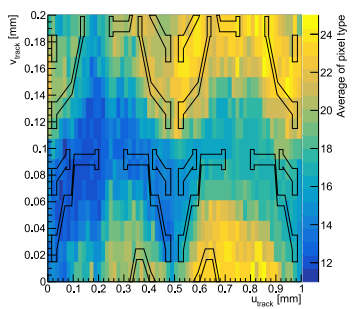

(e)

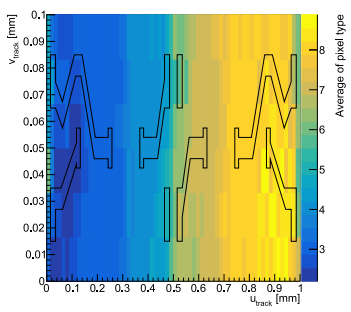

(c)

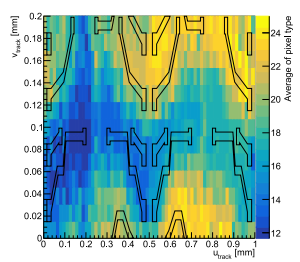

(f)

Figure A.17.: Average prototype pixel number of the hit pixel at the position of the matched track folded into rectangular (first row) and hexagonal (second row) super-cell, at $500 \mathrm{~V}, 600 \mathrm{~V}$ with beam energy $4.0 \mathrm{GeV}, 600 \mathrm{~V}$ with beam energy $4.6 \mathrm{GeV}$ (left to right). 


\section{Charge Collection}

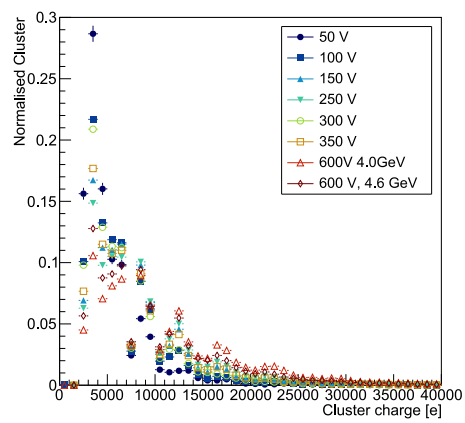

Figure A.18.: Cluster charge of hits in the planar diamond detector for different bias voltages. Distributions normalised to number of clusters.

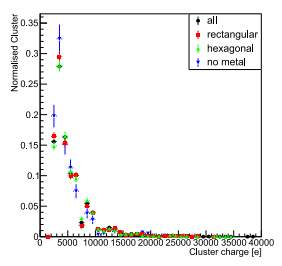

(a)

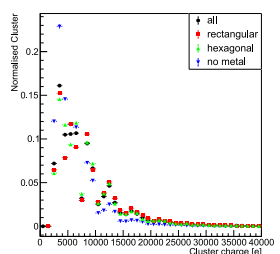

(e)

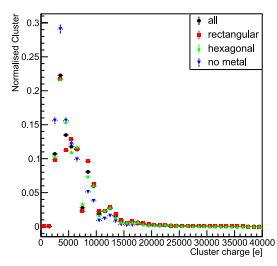

(b)

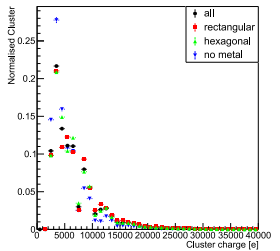

(f)

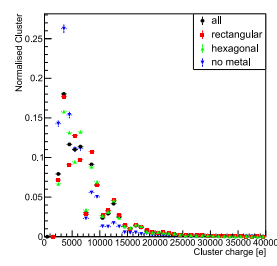

(c)

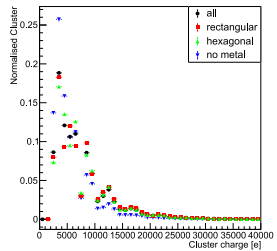

$(\mathrm{g})$

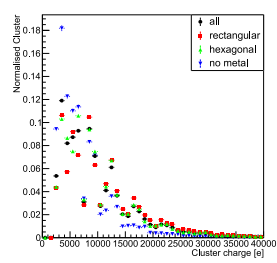

(d)

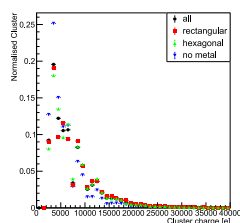

(h)

Figure A.19.: Cluster charge distributions for different regions of the planar diamond, at $50 \mathrm{~V}, 100 \mathrm{~V}, 150 \mathrm{~V}, 200 \mathrm{~V}$ (first row), and $250 \mathrm{~V}, 300 \mathrm{~V}, 350 \mathrm{~V}, 400 \mathrm{~V}$ (second row). 


\section{A. Appendix}

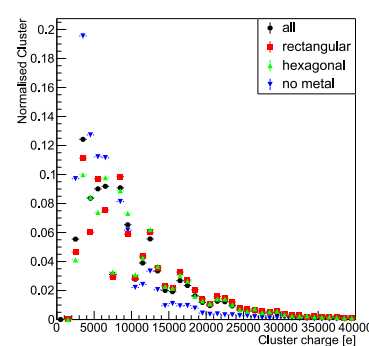

(a)

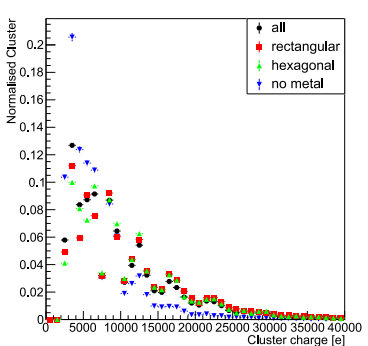

(b)

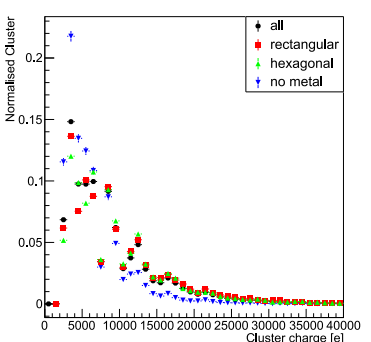

(c)

Figure A.20.: Cluster charge distributions for different regions of the planar diamond, at $500 \mathrm{~V}, 600 \mathrm{~V}$ with beam energy $4.0 \mathrm{GeV}, 600 \mathrm{~V}$ with beam energy $4.6 \mathrm{GeV}$ (left to right).

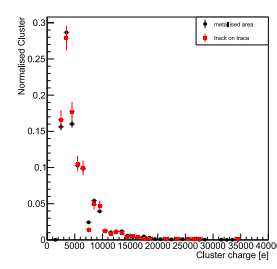

(a)

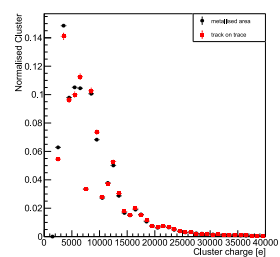

(e)

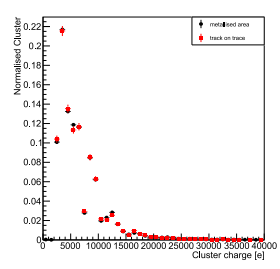

(b)

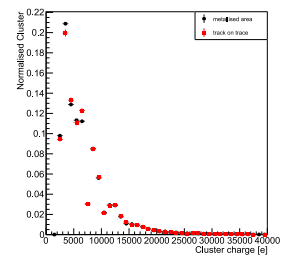

(f)

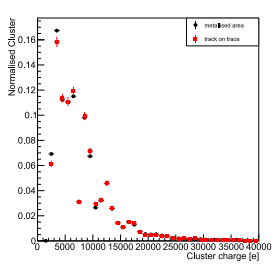

(c)

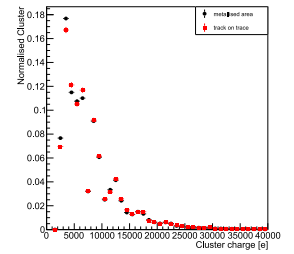

(g)

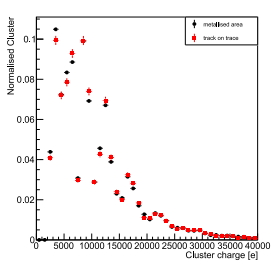

(d)

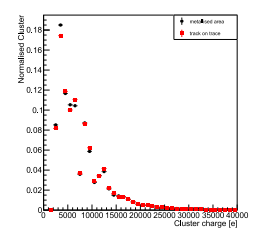

(h)

Figure A.21.: Cluster charge distributions compared to cluster charge for track on trace, at $50 \mathrm{~V}, 100 \mathrm{~V}, 150 \mathrm{~V}, 200 \mathrm{~V}$ (first row), and $250 \mathrm{~V}, 300 \mathrm{~V}, 350 \mathrm{~V}, 400 \mathrm{~V}$ (second row). 


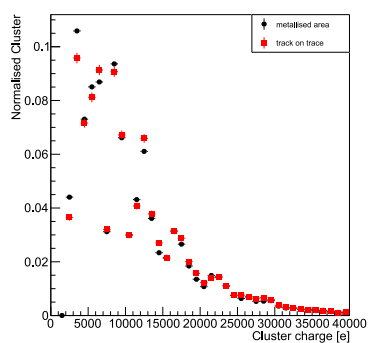

(a)

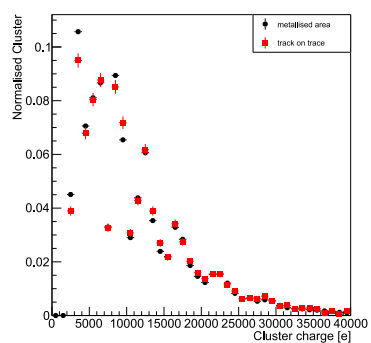

(b)

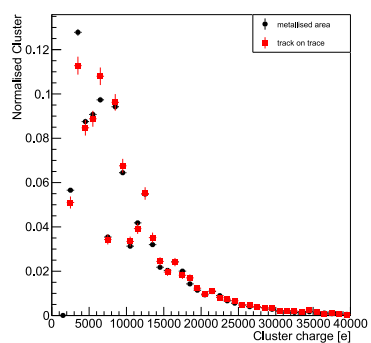

(c)

Figure A.22.: Cluster charge distributions compared to cluster charge for track on trace, at $500 \mathrm{~V}, 600 \mathrm{~V}$ with beam energy $4.0 \mathrm{GeV}, 600 \mathrm{~V}$ with beam energy $4.6 \mathrm{GeV}$ (left to right).

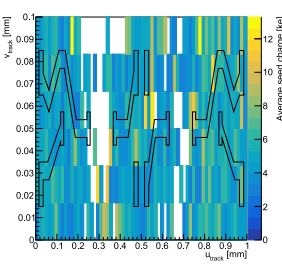

(a)

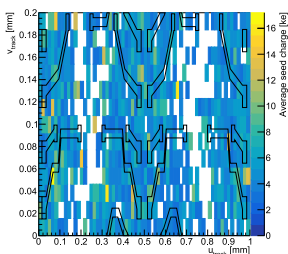

(e)

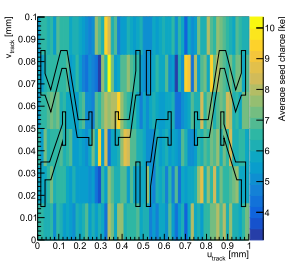

(b)

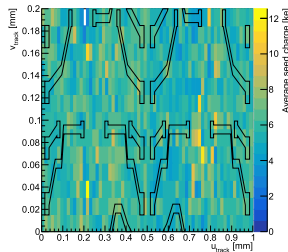

(f)

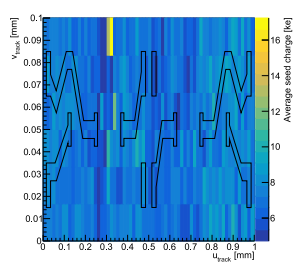

(c)

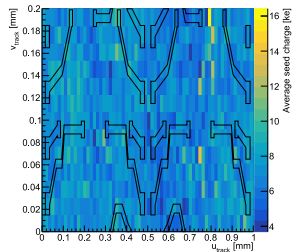

(g)

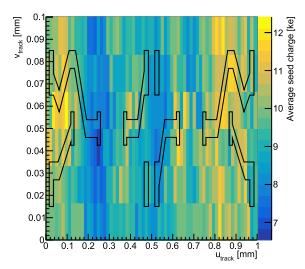

(d)

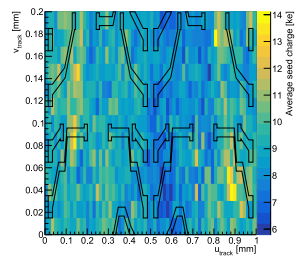

(h)

Figure A.23.: Average seed charge of the hit pixel at the position of the matched track folded into the super-cell of the rectangular (first row) and hexagonal region (second row), at $50 \mathrm{~V}, 100 \mathrm{~V}, 150 \mathrm{~V}, 200 \mathrm{~V}$ (from left to right). 


\section{A. Appendix}

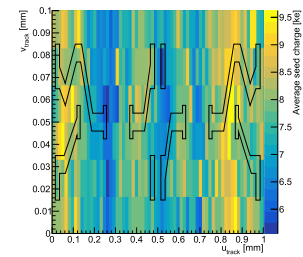

(a)

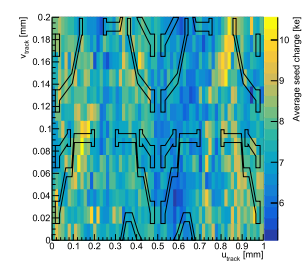

(e)

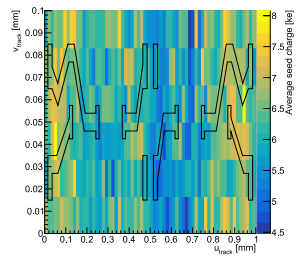

(b)

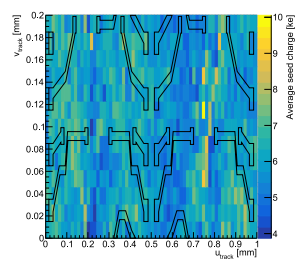

(f)

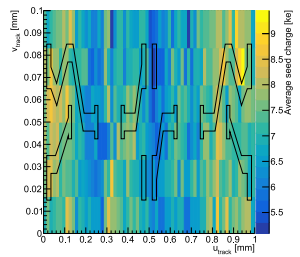

(c)

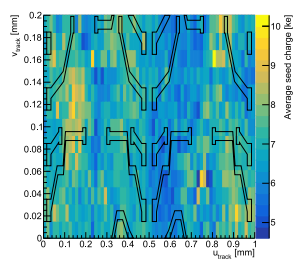

$(\mathrm{g})$

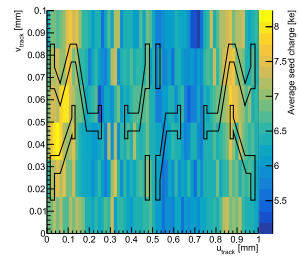

(d)

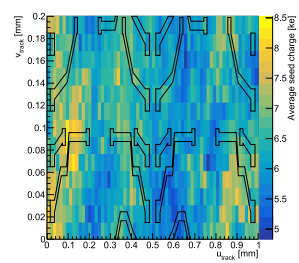

(h)

Figure A.24.: Average seed charge of the hit pixel at the position of the matched track folded into the super-cell of the rectangular (first row) and hexagonal region (second row), at $250 \mathrm{~V}, 300 \mathrm{~V}, 350 \mathrm{~V}, 400 \mathrm{~V}$ (left to right).

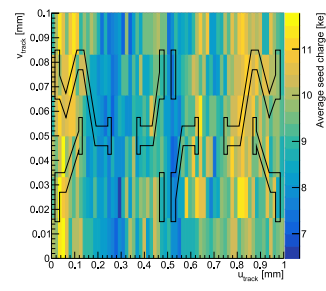

(a)

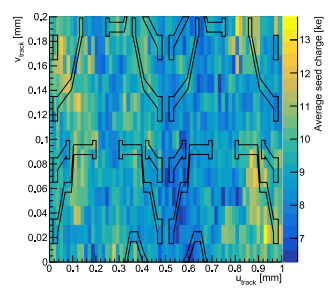

(d)

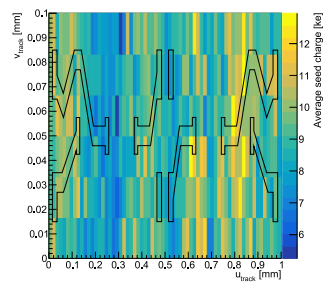

(b)

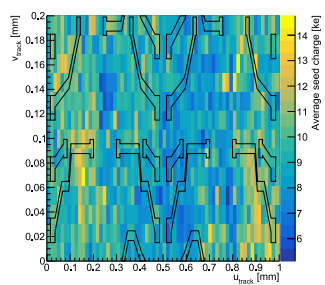

(e)

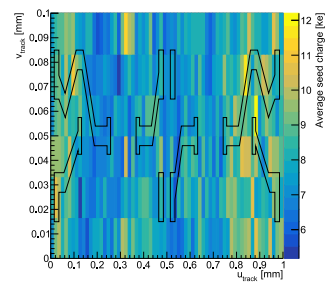

(c)

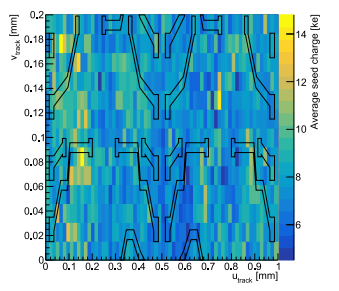

(f)

Figure A.25.: Average seed charge of the hit pixel at the position of the matched track folded into the super-cell of the rectangular (first row) and hexagonal region (second row), at $500 \mathrm{~V}, 600 \mathrm{~V}$ with beam energy $4.0 \mathrm{GeV}, 60 \mathrm{~V}$ with beam energy $4.6 \mathrm{GeV}$ (left to right). 


\section{Efficiency}

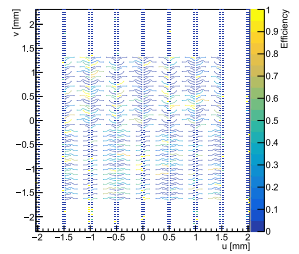

(a)

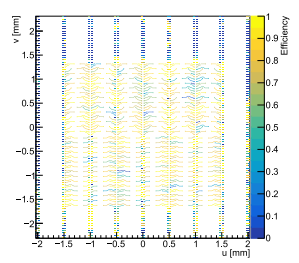

(e)

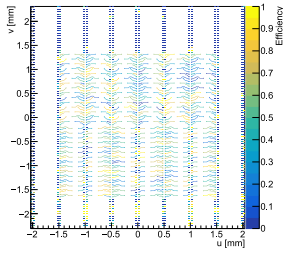

(b)

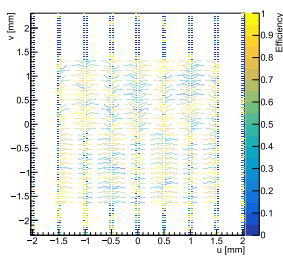

(f)

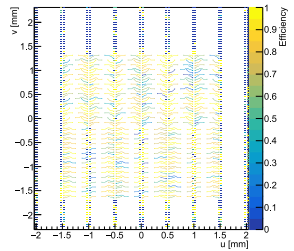

(c)

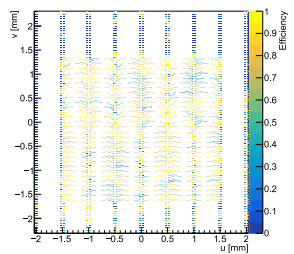

$(\mathrm{g})$

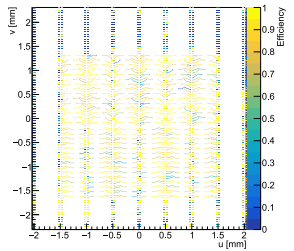

(d)

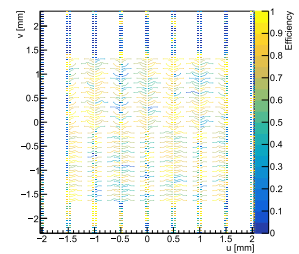

(h)

Figure A.26.: Map of the efficiency for expected tracks intersecting traces displayed in the trace, at $50 \mathrm{~V}, 100 \mathrm{~V}, 150 \mathrm{~V}, 200 \mathrm{~V}$ (first row) and $250 \mathrm{~V}, 300 \mathrm{~V}, 350 \mathrm{~V}$, $400 \mathrm{~V}$ (second row).

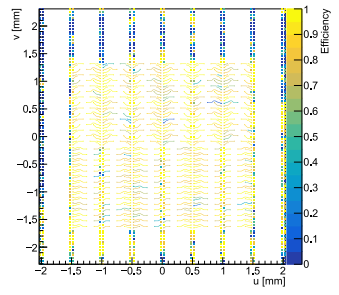

(a)

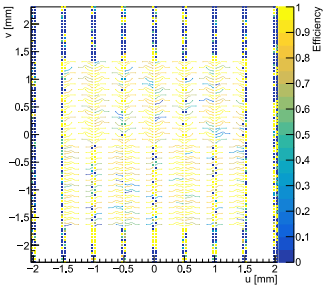

(b)

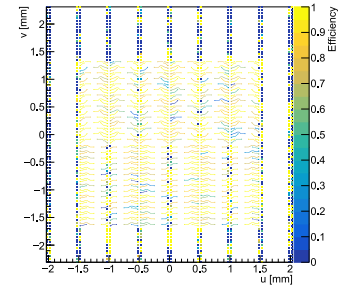

(c)

Figure A.27.: Map of the efficiency for expected tracks intersecting traces displayed in the trace, at $500 \mathrm{~V}, 600 \mathrm{~V}$ with beam energy $4.0 \mathrm{GeV}, 600 \mathrm{~V}$ with beam energy $4.6 \mathrm{GeV}$ (left to right). 


\section{A. Appendix}

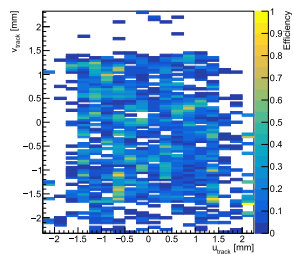

(a)

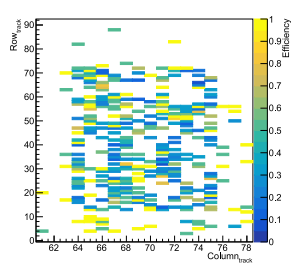

(e)

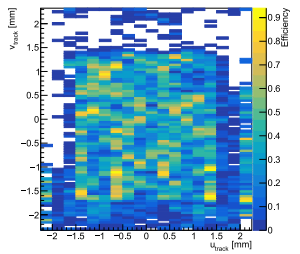

(b)

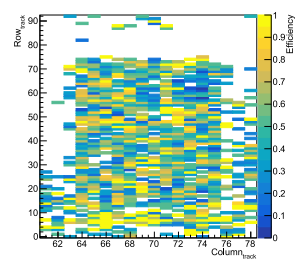

(f)

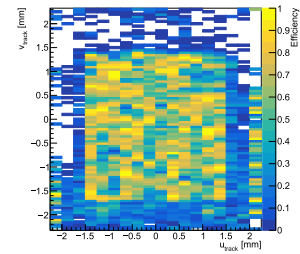

(c)

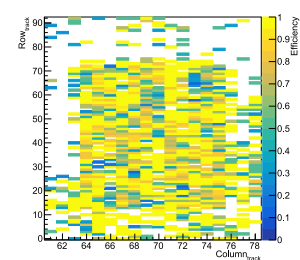

(g)

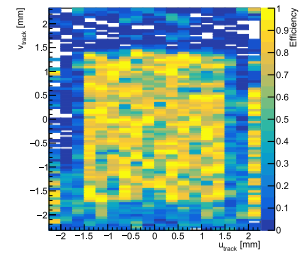

(d)

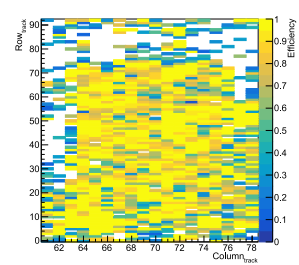

(h)

Figure A.28.: Map of the efficiency for all expected tracks in the diamond (first row), and map of the efficiency for expected tracks intersecting traces displayed in the read-out pixel column and row associated to the trace (second row), at $50 \mathrm{~V}, 100 \mathrm{~V}, 150 \mathrm{~V}, 200 \mathrm{~V}$ (left to right).

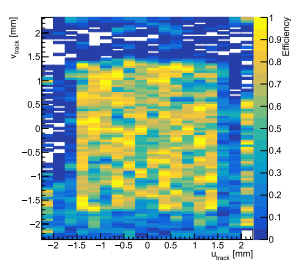

(a)

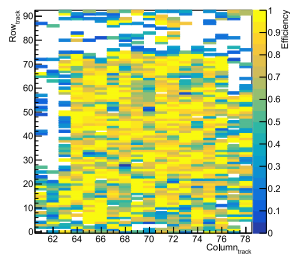

(e)

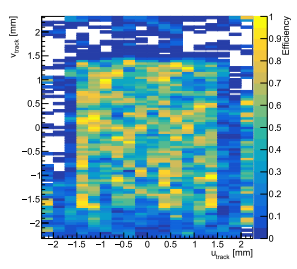

(b)

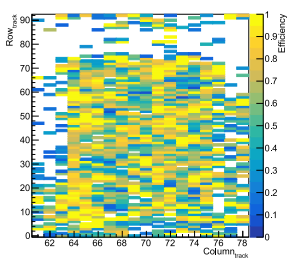

(f)

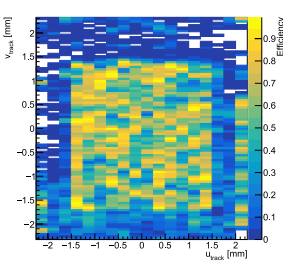

(c)

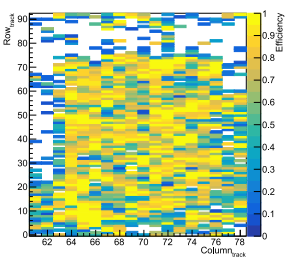

(g)

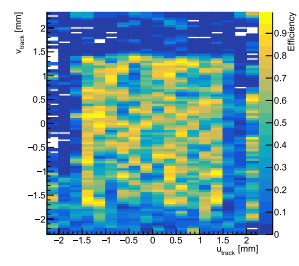

(d)

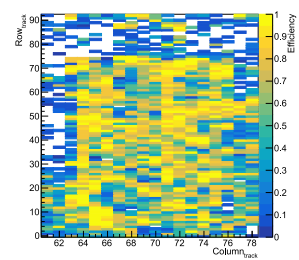

(h)

Figure A.29.: Map of the efficiency for all expected tracks in the diamond (first row), and map of the efficiency for expected tracks intersecting traces displayed in the read-out pixel column and row associated to the trace (second row), at $250 \mathrm{~V}, 300 \mathrm{~V}, 350 \mathrm{~V}, 400 \mathrm{~V}$ (left to right). 


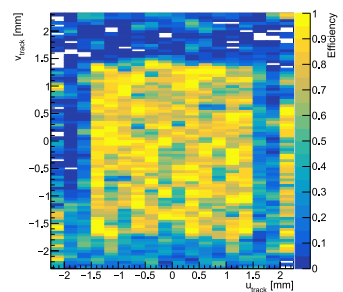

(a)

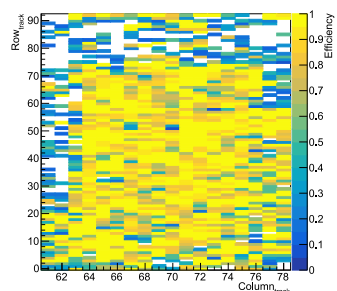

(d)

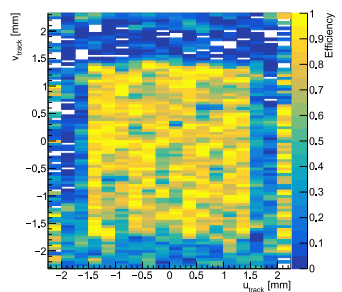

(b)

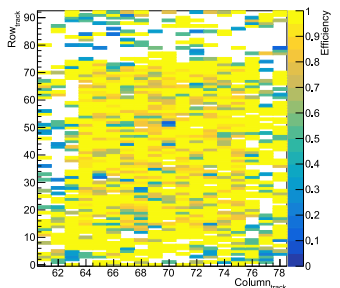

(e)

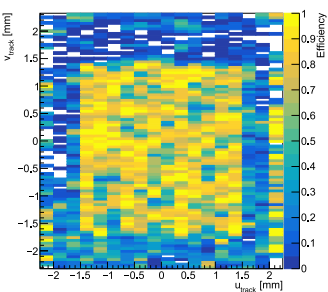

(c)

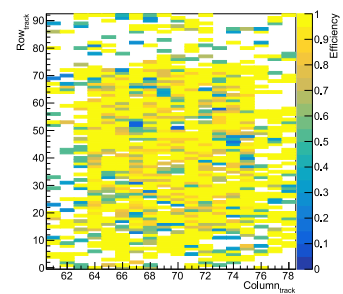

(f)

Figure A.30.: Map of the efficiency for all expected tracks in the diamond (first row), and map of the efficiency for expected tracks intersecting traces displayed in the read-out pixel column and row associated to the trace (second row), at $500 \mathrm{~V}, 600 \mathrm{~V}$ with beam energy $4.6 \mathrm{GeV}, 600 \mathrm{~V}$ with beam energy $4.6 \mathrm{GeV}$ (left to right).

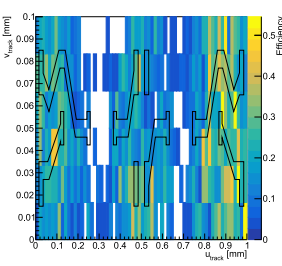

(a)

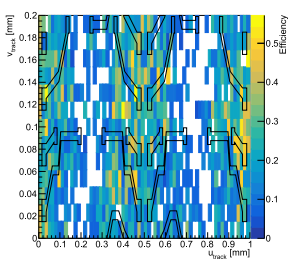

(e)

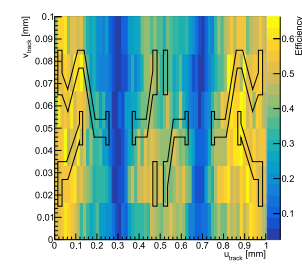

(b)

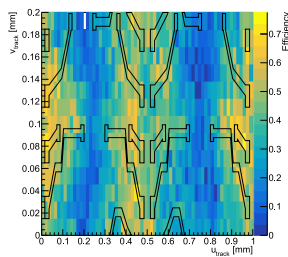

(f)

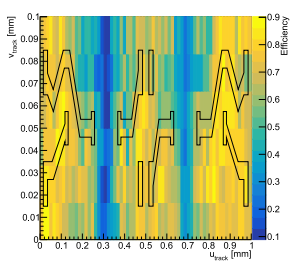

(c)

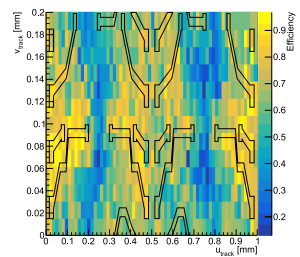

(g)

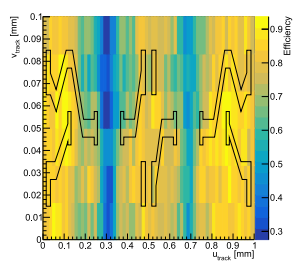

(d)

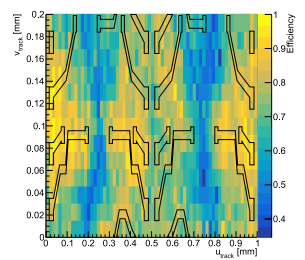

(h)

Figure A.31.: Efficiency at the track position folded into the super-cell of the rectangular (first row) and hexagonal region (second row), at $50 \mathrm{~V}, 100 \mathrm{~V}, 150 \mathrm{~V}, 200 \mathrm{~V}$ (left to right). 


\section{A. Appendix}

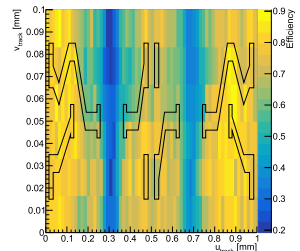

(a)

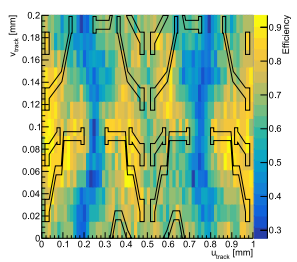

(e)

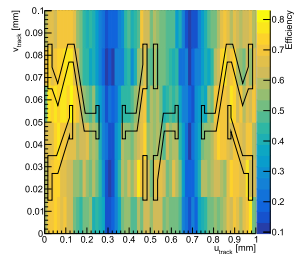

(b)

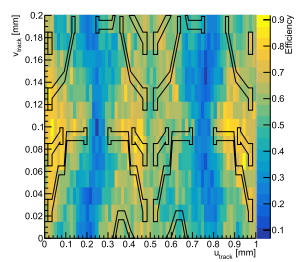

(f)

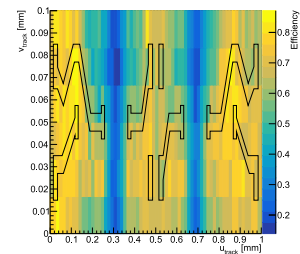

(c)

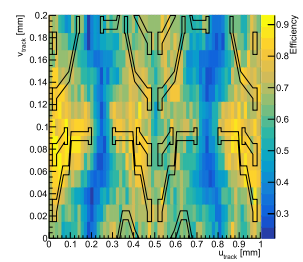

(g)

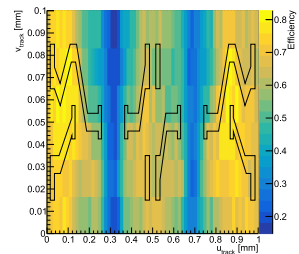

(d)

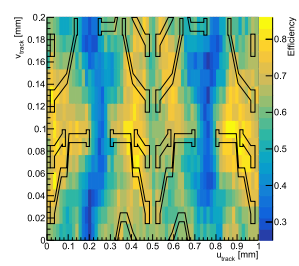

(h)

Figure A.32.: Efficiency at the track position folded into the super-cell of the rectangular (first row) and hexagonal region (second row), at $250 \mathrm{~V}, 300 \mathrm{~V}, 350 \mathrm{~V}$, $400 \mathrm{~V}$ (left to right).

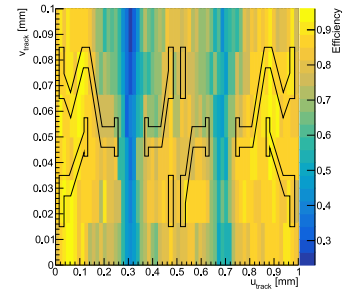

(a)

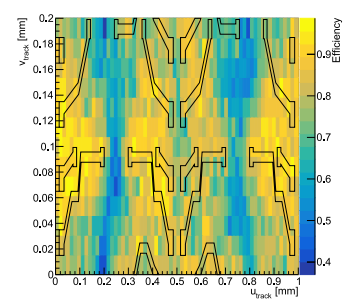

(d)

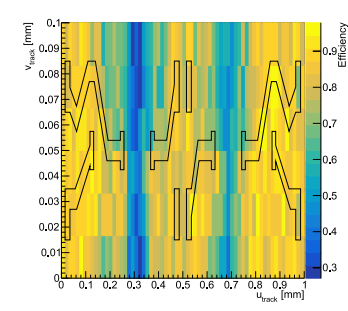

(b)

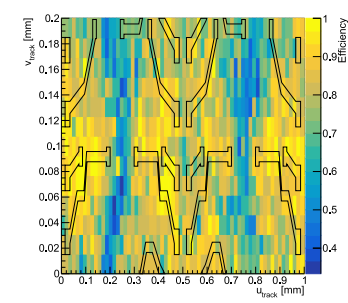

(e)

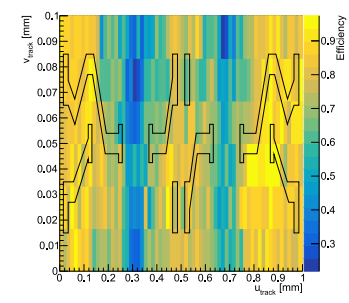

(c)

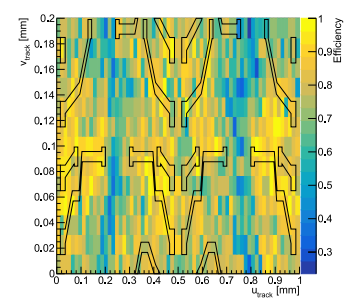

(f)

Figure A.33.: Efficiency at the track position folded into the super-cell of the rectangular (first row) and hexagonal region (second row), at $500 \mathrm{~V}, 600 \mathrm{~V}$ with beam energy $4.0 \mathrm{GeV}, 600 \mathrm{~V}$ with beam energy $4.6 \mathrm{GeV}$ (left to right). 
A.2. Test Beam Measurements with Diamond Pixel Detectors

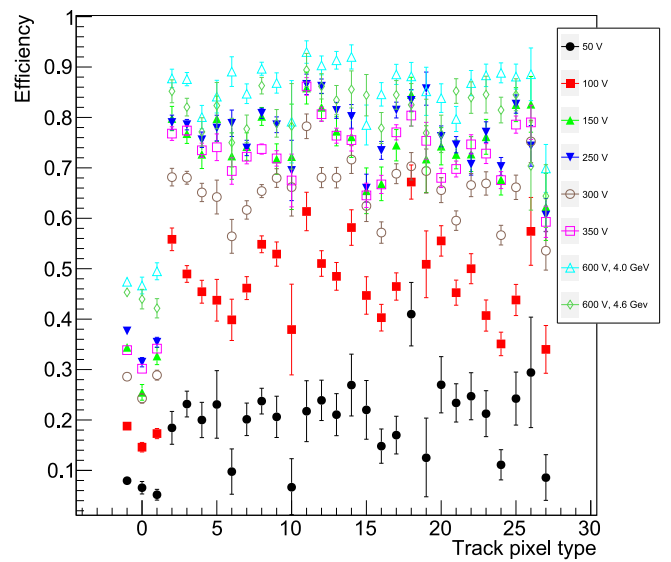

Figure A.34.: Efficiency of the prototype pixels for tracks intersecting traces. 


\section{A. Appendix}

\section{A.2.3. 3D Diamond}

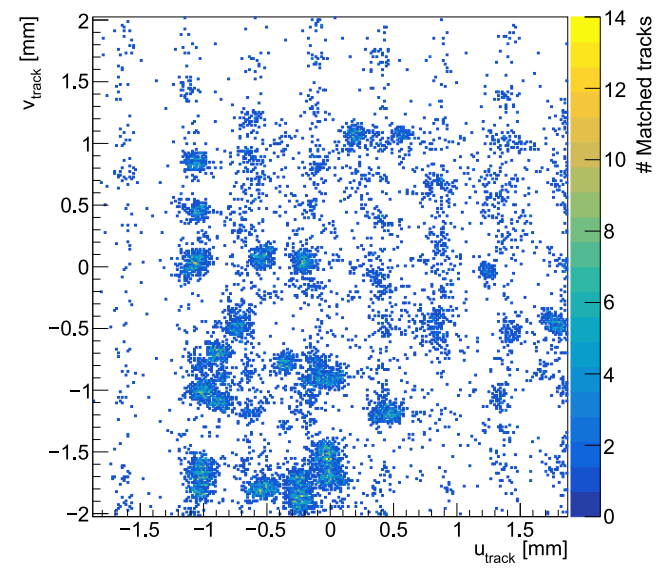

(a)

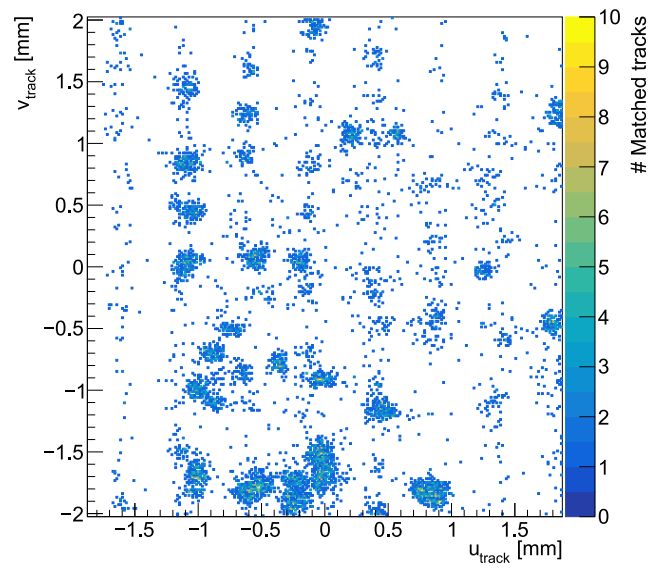

(b)

Figure A.35.: Matched track incidents positions in the sensitive area of the 3D diamond module, biased with $50 \mathrm{~V}$ and $70 \mathrm{~V}$ in (a) and (b), respectively.

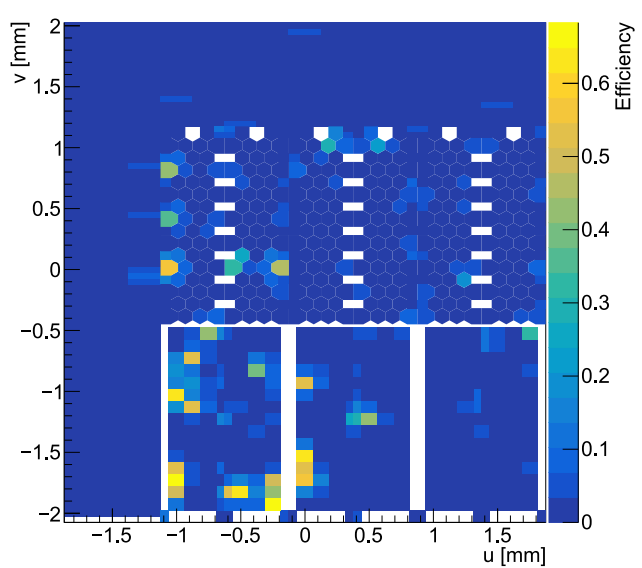

(a)

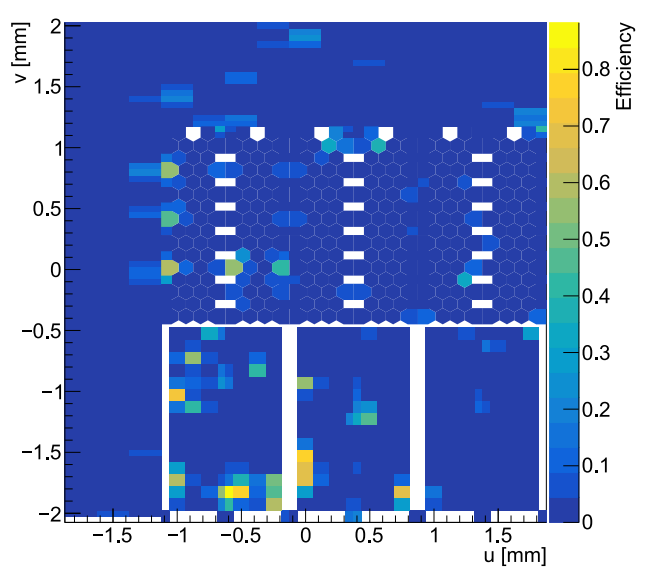

(b)

Figure A.36.: Efficiency per pixel mapped to the layout of the 3D diamond module, biased with $50 \mathrm{~V}$ and $70 \mathrm{~V}$ in (a) and (b), respectively. 